\title{
The highs and lows of drugs use: acute effects of cannabis, cocaine and MDMA on impulsivity, cognition and subjective experience
}

Citation for published version (APA):

van Wel, J. H. P. (2014). The highs and lows of drugs use: acute effects of cannabis, cocaine and MDMA on impulsivity, cognition and subjective experience. [Doctoral Thesis, Maastricht University]. Maastricht University. https://doi.org/10.26481/dis.20140328jw

Document status and date:

Published: 01/01/2014

DOI:

10.26481/dis.20140328jw

Document Version:

Publisher's PDF, also known as Version of record

Please check the document version of this publication:

- A submitted manuscript is the version of the article upon submission and before peer-review. There can be important differences between the submitted version and the official published version of record.

People interested in the research are advised to contact the author for the final version of the publication, or visit the DOI to the publisher's website.

- The final author version and the galley proof are versions of the publication after peer review.

- The final published version features the final layout of the paper including the volume, issue and page numbers.

Link to publication

\footnotetext{
General rights rights.

- You may freely distribute the URL identifying the publication in the public portal. please follow below link for the End User Agreement:

www.umlib.nl/taverne-license

Take down policy

If you believe that this document breaches copyright please contact us at:

repository@maastrichtuniversity.nl

providing details and we will investigate your claim.
}

Copyright and moral rights for the publications made accessible in the public portal are retained by the authors and/or other copyright owners and it is a condition of accessing publications that users recognise and abide by the legal requirements associated with these

- Users may download and print one copy of any publication from the public portal for the purpose of private study or research.

- You may not further distribute the material or use it for any profit-making activity or commercial gain

If the publication is distributed under the terms of Article $25 \mathrm{fa}$ of the Dutch Copyright Act, indicated by the "Taverne" license above, 


\section{The highs and lows of drug use:}

Acute effects of cannabis, cocaine and MDMA on impulsivity, cognition and subjective experience 


\section{Colofon}

(C) J.H.P. van Wel, Maastricht

Cover illustration Mindy Frijns

Production Proefschriftmaken.n

ISBN

978-90-8891-833-9 


\title{
The highs and lows of drug use:
}

Acute effects of cannabis, cocaine and MDMA on impulsivity, cognition and subjective experience

\author{
PROEFSCHRIFT \\ ter verkrijging van de graad van doctor aan de Universiteit Maastricht, \\ op gezag van de Rector Magnificus, Prof dr. L.L.G. Soete \\ volgens het besluit van het College van Decanen, \\ in het openbaar te verdedigen \\ op vrijdag 28 maart 2014 om 14.00 uur
}

door

Janelle Henrica Petronelle van Wel 


\section{Promotor}

Prof.dr. J.G. Ramaekers

\section{Copromotores}

Dr. K.P.C. Kuypers

Dr. R.J. Verkes (Radboud Universiteit Nijmegen)

\section{Beoordelingscommissie}

Prof. dr. W.J. Riedel (voorzitter)

Dr. A. Blokland

Prof. dr. R. Cools (Radboud Universiteit Nijmegen)

Prof. dr. B. Hommel (Universiteit Leiden)

Dr. C. Nederkoorn 


\section{TABLE OF CONTENTS}

Chapter 1 General introduction

Chapter 2 Single doses of THC and cocaine decrease proficiency of impulse control in heavy cannabis users

Chapter 3 Trait impulsivity contributes to psychedelic experience of cannabis and cocaine use

Chapter $4 \quad$ Reward related brain activity and task performance during cannabis and cocaine intoxication

Chapter $5 \quad$ Blockade of $5-\mathrm{HT}_{2}$ receptor selectively prevents MDMA induced verbal memory impairment

Chapter 6 Effects of acute MDMA intoxication on mood and impulsivity: role of the $5-\mathrm{HT}_{2}$ and $5-\mathrm{HT}_{1}$ receptors 89

$\begin{array}{lll}\text { Chapter } 7 & \text { General discussion } & 105\end{array}$

$\begin{array}{ll}\text { References } & 119\end{array}$

$\begin{array}{ll}\text { Summary } & 143\end{array}$

$\begin{array}{ll}\text { Samenvatting } & 149\end{array}$

$\begin{array}{ll}\text { Dankwoord } & 155\end{array}$

$\begin{array}{ll}\text { Curriculum Vitae } & 159\end{array}$

$\begin{array}{ll}\text { Publications } & 161\end{array}$ 



\section{Chapter 1}

GENERAL INTRODUCTION 


\section{GENERAL INTRODUCTION}

A common definition for drugs of abuse is any drug taken for nonmedical reasons, usually for mind-altering effects. Drug use can vary from the consumption of cannabinoids such as marihuana and hashish to stimulants like cocaine and amphetamines, as well as the use of opioids and hallucinogens. Three of the most frequently used drugs are cannabis, cocaine and 3,4-Methylenedioxymethamphetamine (MDMA, ecstasy). Estimates of lifetime cannabis use range from $23.7 \%$ in 15-64-year-olds in the European Union (EU (EMCDDA, 2012)) to $51.9 \%$ of young adults aged 18-25 in the United States of America (USA, (SAMHSA, 2012)). In the EU, an estimated 1\% (EMCDDA, 2012) of the entire population is using cannabis daily, and intensive patterns of use can be quite common, especially in young males. Cocaine remains the second most commonly used illicit drug in the EU, where an estimated $4.6 \%$ (EMCDDA, 2012) of all inhabitants have used the drug at least once in their lifetime. MDMA has been used at least once in their lifetime by an average of $4.5 \%$ of European adults, mainly at parties or other social events (EMCDDA, 2012; Murphy, Wareing, \& Fisk, 2006).

\section{Background information}

\section{MDMA}

MDMA is a ring-substituted phenethylamine, structurally similar to methamphetamine, was synthesized in 1912 by pharmaceutical company Merck as a precursor for an antihemorrhagic medication. However, the first studies in humans did not take place until the late 1970s (Bernschneider-Reif, Oxler, \& Freudenmann, 2006).

MDMA is usually taken in the form of tablets, which studies have shown to contain 3$104 \mathrm{mg}$ of the active substance, although pills containing more than $130 \mathrm{mg}$ have also been reported (EMCDDA, 2012). Users typically ingest more than one tablet per occasion of use, making it difficult to correctly assess long-term effects of the drug due to considerable interpersonal variability in exposure to the drug (Murphy et al., 2006). Following oral ingestion, the maximum value of the drug in blood $\left(t_{\max }\right)$ is usually reached within 2 hours (de la Torre et al., 2004).

The main mechanism of action of MDMA is through potent release and/or reuptake inhibition of presynaptic serotonin $(5-\mathrm{HT})$, thereby causing a massive influx of these neurotransmitters into the synapse and post-synaptic neuron. MDMA also has a direct effect 
on a number of receptors, such as the $5-\mathrm{HT}_{2 \mathrm{~A}}$ receptor, $\mathrm{M}_{1}$ receptor, the $\alpha_{2}$ receptor and the $\mathrm{H}_{1}$ receptor (de la Torre et al., 2004; Gudelsky \& Yamamoto, 2008).

\section{Cannabis}

The cannabis sativa plant is an annual herbaceous plant of the Cannabaceae family, often used for its psychoactive effects. The flowering tops can be dried (weed), pressed into resin (hashish), or distilled into oil. Most users typically smoke the drug, often combining weed or hashish with tobacco, but other methods of inhalation, as well as consuming weed in food or in a drink, also occur (Netherlands Institute of Mental Health and Addiction, 2012; United Nations Office on Drugs and Crime, 2012). After smoking, peak plasma concentrations of THC $\left(t_{\max }\right)$ are reached almost instantly. Out of several cannabinoids present in cannabis, $\Delta 9$ tetrahydrocannabinol (THC) is the most studied.

The acute effects of cannabis are mediated by two types of receptors, the $\mathrm{CB}_{1}$ and $\mathrm{CB}_{2}$-receptors, both of which are active in the brain. The prevalence of the $\mathrm{CB}_{1}$ receptor is highest in the hippocampus, cerebellum and striatum. The exact role of the $\mathrm{CB}_{2}$ receptor is as of yet unknown, but it mainly seems to play a role in neuroinflammatory conditions.

The cannabinoid system (CS) has been shown to increase the activity of dopaminergic (DA) neurons in the ventral tegmental area and the mesolimbic pathway, as well as in the prefrontal cortex (Ameri, 1999; Benito et al., 2008; Melis \& Pistis, 2007). However, the effects of chronic cannabis administration on DA levels are not as clear cut. It has been shown that after acute administration of cannabis in heavy users, levels of DA either increase (Bossong et al., 2009), decrease (Verrico, Jentsch, \& Roth, 2003) or do not change at all (Stokes, Mehta, Curran, Breen, \& Grasby, 2009).

\section{Cocaine}

Cocaine is a hydrochloric salt is made by converting leaves from the coca plant (Erythroxylon coca) into this basic form (Stolberg, 2011). The coca plant is the only known natural source of cocaine and is almost exclusively planted in the Andes in South-America, and as such has been used as a ceremonial plant for thousands of years. Although occasionally used for medicinal purposes, cocaine is mainly known as a stimulant drug. It is typically snorted in powder form, or smoked (crack), while injection is less common (European Monitoring 
Centre for Drugs and Drug Addiction, 2010). If snorted nasally, cocaine reaches maximal plasma concentrations after 10 minutes (Fattinger, Benowitz, Jones, \& Verotta, 2000).

Cocaine exerts its effects on the brain by inhibiting the dopamine transporter (DAT), thereby increasing the levels of DA in reward circuits and activating mesolimbic and mesocortical DA pathways (Breiter et al., 1997; Kufahl et al., 2005; Volkow, Fowler, Wang, \& Swanson, 2004).

\section{Drugs of abuse and impulsivity}

The definition of impulsivity as a unitary construct has been difficult. The most common definition is 'acting without forethought', but research has shown that different forms of impulsivity can be dissociated at the neuroanatomical and behavioral level. As such, impulsivity has come to be defined in at least three distinct ways: impaired inhibition of behavior, lack of regard for the consequences of one's actions and an inability to delay reward (Dalley, Everitt, \& Robbins, 2011; Pattij \& Vanderschuren, 2008).

One region that is strongly associated with inhibitory control and decision making is the prefrontal cortex (PFC), which receives input from the limbic circuit. After drug administration, the level of dopamine in limbic regions increases, which causes the rewarding feeling (the 'high' of a drug) (Volkow, Wang, Fowler, \& Tomasi, 2012). Somewhere in the transition from drug use to addiction, the inhibitory function of the PFC gets dysregulated, while output from the limbic region is increased. This results in the decrease in impulse control and the increase in impulse generation often seen in addicts (Goldstein \& Volkow, 2002). Therefore, contemporary research has focused mainly on dopaminergic modulation of impulse control. Several studies have shown that a decrease in dopamine levels make someone more likely to lose control over their impulses. For example, studies in recreational cannabis users have shown that acute intoxication with cannabis can lead to decreases in impulse control (McDonald, Schleifer, Richards, \& de Wit, 2003; Ramaekers et al., 2006; Ramaekers, Kauert, Theunissen, Toennes, \& Moeller, 2009), which could be due to acute decreases in DA levels. However, studies in experienced cannabis users are more conflicting. Some studies show no effect, indicating that tolerance to the effect of the drug on impulsivity measures occurs (Ramaekers et al., 2011), while in other studies heavy users are negatively affected by cannabis on tasks measuring impulse control (Ramaekers, Kauert, et al., 2009). It is therefore important to assess whether this tolerance occurs in all 
neuropsychological domains (such as memory, impulsivity and psychomotor functioning) or only in a specific subdomain. On the other hand, administering dopamine-enhancing drugs like amphetamines or cocaine can increase impulse control. Evidence for this viewpoint comes from studies investigating response inhibition (Fillmore, Rush, \& Hays, 2005, 2006; Garavan, Kaufman, \& Hester, 2008) after cocaine administration. In these studies, compared to placebo, either no difference or an amelioration of impulse control was shown during cocaine intoxication.

Impulse control may also depend on baseline dopamine levels. Persons with a higher baseline dopamine level are more likely to have higher impulsivity levels than persons with lower baseline dopamine levels (Congdon \& Canli, 2008). Furthermore, studies on the effects of amphetamines indicate that drugs that work on the DA system can affect ratings of mood and that this effect is even stronger when subjects score high on measures of trait impulsivity (Kirkpatrick, Johanson, \& de Wit, 2013; Oswald et al., 2007). This suggests that higher impulsive persons are more susceptible to the subjective effects of dopamineenhancing drugs, and might therefore be more likely to develop an addiction through increases in the rewarding feelings of those drugs.

Although the main focus in impulsivity research has been on dopamine, a role for the serotonin (5-hydroxytryptamine; 5-HT) system in controlling impulsive behavior has also been shown (Kirby, Zeeb, \& Winstanley, 2011). Not only have low levels of 5-HT been implicated in impulse control disorders, direct manipulation of 5-HT levels has shown that a shortage of 5-HT can lead to an increase in impulsive behavior, while a surplus has no effect (Cools, Roberts, \& Robbins, 2008; Young \& Leyton, 2002). Experiments in rats have suggested a role for the $5-\mathrm{HT}_{1}$ and $5-\mathrm{HT}_{2}$ receptors in impulse control (Anastasio et al., 2011; Hadamitzky \& Koch, 2009; Robinson et al., 2008; Schreiber \& de Vry, 1993), but it is not yet known whether the same goes for humans. It is especially important to know which receptor subtype is involved in impulse control, since this could influence research for drug targets targeting those subtypes in order to treat disorders associated with deficits in impulse control. For example, the $5-\mathrm{HT}_{2 \mathrm{~A}}$ receptor is implied in regulating glutamatergic control of the prefrontal cortex (Robinson et al., 2008), while $5-\mathrm{HT}_{1}$ receptors are more prevalent in structures of the dopamine-mediated limbic system (Müller, Carey, Huston, \& De Souza Silva, 2007). Nonetheless, despite the evidence for the involvement of the 5-HT system in impulse control, the influence of acute administration of MDMA on impulsive behavior in 
humans remains unclear. MDMA intoxication has been shown to either have no effect on impulse control or to decrease impulsive behavior (Bosker, Kuypers, Conen, \& Ramaekers, 2010; Kuypers, Wingen, Samyn, Limbert, \& Ramaekers, 2007; Ramaekers \& Kuypers, 2006). Further elucidation on the effects of MDMA on impulse control and the involvement of the 5-HT system is therefore necessary.

\section{Drugs of abuse and cognition}

\section{Psychomotor functioning}

The term psychomotor function is used to indicate the interplay between sensorimotor and cognitive processes, in which the mesolimbic and nigrostriatal dopamine systems have been shown to play a significant role (Canales \& Iversen, 2000).

Persons under the influence of cocaine are more aroused, and therefore one would expect them to respond faster during a psychomotor task. However, not all studies investigating the acute effects of cocaine on reaction speed have shown this effect. Previous work indicates that cocaine either has no effect or improves psychomotor speed, while keeping the number of errors at a level similar to placebo (Haney, Hart, Collins, \& Foltin, 2005; Higgins et al., 1990; Johnson et al., 1998; Stillman, Jones, Moore, Walker, \& Welm, 1993). On the other hand, when cannabis is administered to recreational drug users, psychomotor functioning (Ménétrey et al., 2005; Ramaekers et al., 2006) has been shown to be affected as indicated by a decrease in accuracy and slower reaction times. This effect is less evident in regular users (Ramaekers et al., 2011; Ramaekers, Kauert, et al., 2009), possibly due to tolerance to the impairing effects of cannabis.

\section{Memory}

There is a wealth of studies showing that disturbances of the serotonin system cause disturbances in memory functions such as learning, consolidation and retrieval of information (Müller et al., 2007). All receptors of the 5-HT family (5-HT 1 through $5-\mathrm{HT}_{7}$ ), but particularly the $5-\mathrm{HT}_{2}$ receptors, have been implicated in memory function. Evidence stems from immunohistological studies, showing that the 5-HT receptors are highly prevalent in the rat hippocampus, the structure most associated with memory consolidation, as well as the amygdala and frontal cortex (Bombardi \& Di Giovanni, 2013; Meneses, 1999; Meneses, 2002). However, how dysfunction of the 5 -HT system can lead to memory impairment is less 
well understood. One way to investigate the function of the 5-HT system is through acute drug studies in which administration of $5 \mathrm{HT}$ agonists is preceded with the administration of $5 \mathrm{HT}$ receptor blockers. If a memory effect occurs after the agonist is given, but this effect is negated by the pretreatment, then this is likely to be caused (at least partially) by that receptor subsystem.

Since MDMA functions as an 5-HT agonist and has been shown to impair performance on verbal learning tasks, prospective memory, spatial memory and continuous recognition (Hasler, Studerus, Lindner, Ludewig, \& Vollenweider, 2009; Kuypers, de la Torre, Farre, Pujadas, \& Ramaekers, 2013; Kuypers \& Ramaekers, 2005, 2007; Kuypers, Wingen, Heinecke, Formisano, \& Ramaekers, 2011; Ramaekers, Kuypers, Wingen, Heinecke, \& Formisano, 2009) the procedure described above can be employed to elucidate the effects of 5-HT receptor agonism or antagonism on memory.

\section{Drugs of abuse and mood}

Decreases in serotonin levels in the brain have been associated with decreases in mood, while increases in serotonin have been shown to do the opposite (Elliott, Zahn, Deakin, \& Anderson, 2011). As MDMA works on the serotonin level, it is not surprising that recreational users report feeling friendly, sociable and euphoric under the influence of MDMA. However, the effect that stands out most is its potential to evoke a state in which users feel exceptionally close to others and experience increased empathy and love. Several experimental studies have shown that acute MDMA intoxication can affect both positive and negative mood states (Dumont \& Verkes, 2006; Hasler, Studerus, Lindner, Ludewig, \& Vollenweider, 2009; Kuypers, de la Torre, Farre, Pujadas, \& Ramaekers, 2013; Kuypers, Wingen, Heinecke, Formisano, \& Ramaekers, 2011; Liechti, Saur, Gamma, Hell, \& Vollenweider, 2000; Liechti \& Vollenweider, 2001; Vollenweider, Liechti, Gamma, Greer, \& Geyer, 2001). So far, studies have mainly suggested a role for the $5-\mathrm{HT}_{2 \mathrm{~A}}$ receptors in MDMA-mediated mood alterations, but there are indications for the involvement of other 5$\mathrm{HT}$ receptor subtypes such as $5-\mathrm{HT}_{1}$ and $5-\mathrm{HT}_{2}$ as well (Landolt \& Wehrle, 2009; Liechti \& Vollenweider, 2001).

Several studies have found an increase as well as a decrease of dopamine to be associated with mood alterations, so he effects of the dopamine system on mood are less clear. During cannabis intoxication, more than half of the users report better sleep, feeling 
more relaxed, altered time perception and an increase in sexual pleasure (Green, Kavanagh, \& Young, 2003). On the other hand, acute cocaine intoxication is characterized by feelings of more euphoria, increased sociability and happiness and heart rate and blood pressure are typically elevated. It can also cause craving for more cocaine and anxious feelings (Davidson, Finch, \& Schenk, 1993; Foltin \& Haney, 2004; Lukas et al., 1996). Despite the knowledge about the emotional experience of recreational users during cannabis or cocaine intoxication, in-depth measurement of mood while under the influence of these drugs has been scarce. Most research to date has focused on the level of 'high' subjects were feeling while intoxicated, while relatively few studies have investigated the direct effect of cannabis or cocaine on subjective experiences. Experimenters that administered cannabis to subjects have found a significant increase in confusion and anxiety (Battistella et al., 2013; D'Souza et al., 2008; Lex, Mendelson, Bavli, Harvey, \& Mello, 1984; Lile, Kelly, \& Hays, 2012), as well as an increase of scores on measures of psychotic experiences (D'Souza et al., 2008; Stokes et al., 2009). Being a stimulant drug, it is no surprise that studies investigating the subjective response to cocaine have mainly found an arousing effect (Penetar et al., 2006; Rush, Baker, \& Wright, 1999; Walsh, Stoops, Moody, \& Bigelow, 2010), but surprisingly little research has been done on other aspects of subjective experience during cocaine intoxication. For example, it is known that cocaine and other stimulants with comparable effects such as dexamphetamine and methylphenidate can increase the likelihood of psychotic symptoms (Curran, Byrappa, \& McBride, 2004; Morton, 1999; Roncero, Ros-Cucurull, Daigre, \& Casas, 2012), but there is little available data of dopaminergic effects on psychotic symptoms in a non-clinical population.

\section{Aim of this dissertation}

Following the previous discussion of problem areas associated with the use of drugs of abuse, the present thesis focuses on impulsivity, mood and cognition during intoxication with three major drugs of abuse: MDMA, cannabis and cocaine. The major research questions include:

1) Do impulsivity, cognition and mood alter during acute intoxication with MDMA, cannabis and cocaine?

2) Does trait impulsivity modulate cannabis and cocaine-induced changes in mood and subjective experience? 
3) Do cannabis and cocaine alter reward anticipation?

4) Does pretreatment with a $5-\mathrm{HT}_{1}$ or a $5-\mathrm{HT}_{2}$ antagonist prevent MDMA induced memory deficits and alterations in impulsivity and mood?

\section{Methodology}

Each study discussed in this dissertation employed an experimental randomized, doubleblind, placebo-controlled within-subjects design. In one study, subjects received $75 \mathrm{mg}$ MDMA, with a pretreatment of ketanserin or pindolol, or placebo. In the other, cannabis (total dose of $450 \mu \mathrm{g} / \mathrm{kg}$ divided over two administrations), cocaine (300 mg) and placebo were administered on separate testing days. All subjects were healthy, experienced drug users.

Performance and mood testing took place around $T_{\max }$ of all drugs. To test impulse control, the stop-signal task, matching familiar figures task and cue-dependent reversal learning task (chapter $\mathbf{2}$ and 6) were used. Mood was appraised with the profile of mood states (POMS) questionnaire (chapter 3 and 6). Furthermore, to test dissociative state and psychedelic experience, the clinician administered dissociative states scale (CADSS) and Bowdle visual analogue scale (B-VAS) were used (chapter 3). Memory was assessed using a word-learning task, spatial memory task and a prospective memory task (chapter 5). Psychomotor performance was measured using the critical tracking task and the divided attention task (chapter 2). Lastly, brain activity was measured using functional magnetic resonance imaging ( $\mathrm{fMRI}$ ) while subjects were performing a monetary incentive delay task (chapter 4).

\section{Outline}

Chapters 2, 3 and 4 describe the effect of cannabis and cocaine on measures of impulse control and mood using both laboratory tests and brain activation. In Chapter 2, a study is described in which the influence of regular cannabis use on impulsivity after acute administration of cannabis and cocaine is investigated. Chapter 3 presents a study focusing on the interaction of trait impulsivity (normal/high) and subjective responses to cannabis or cocaine using measures of mood, dissociative state and psychedelic experience. In order to examine the effect of cannabis or cocaine on the brain during impulse control tasks, the 
study described in chapter 4 uses functional brain imaging to study whether cocaine and cannabis differentially influence reward mechanisms both behaviorally and in the brain.

In chapter 5 and chapter 6 the roles of the $5-\mathrm{HT}_{1}$ and $5-\mathrm{HT}_{2}$ receptors in the effects of MDMA on memory, impulsivity and mood are discussed.

Finally, in chapter $\mathbf{7}$ the main findings of the studies described in the previous chapters are integrated and discussed. Clinical recommendations and suggestions for further studies are presented. 
$18 \mid$ 


\section{CHAPTER 2}

\section{SINGLE DOSES OF THC AND COCAINE DECREASE PROFICIENCY OF} IMPULSE CONTROL IN HEAVY CANNABIS USERS 


\section{Summary}

Cannabis is the most popular drug used in the European Union, closely followed by cocaine. Whereas cannabis has been shown to impair neurocognitive function in occasional cannabis users, such impairments appear less prominent in heavy users, possibly as a result of tolerance. The present study was designed to assess whether the impairing effects of THC in heavy cannabis users would present in a wide range of neuropsychological functions or selectively pertain to specific performance domains. A second aim was to assess the acute effects of cocaine on neurocognitive functions of heavy cannabis users. Heavy cannabis users, who had a history of cocaine use $(\mathrm{N}=61)$, participated in a double-blind, placebo controlled, 3-way crossover study. Subjects received single doses of cocaine $\mathrm{HCl} 300 \mathrm{mg}$, cannabis (THC $300 \mu \mathrm{g} / \mathrm{kg}$ ) and placebo and completed a number of tests measuring impulse control and psychomotor function. Results showed that single doses of cannabis impaired psychomotor function and increased response errors during impulsivity tasks. Single doses of cocaine improved psychomotor function and decreased response time in impulsivity tasks at a cost of making more errors. Heavy cannabis users display impairments in a broad range of neuropsychological domains during THC intoxication. Impairments observed in psychomotor tasks, but not in impulsivity tasks, appeared smaller in magnitude as compared to those previously reported in occasional cannabis users. Heavy cannabis users were sensitive to the stimulatory and inhibitory effects of cocaine on psychomotor function and impulsivity respectively. The reduction in proficiency in impulse control may put drug users at increased risk of repeated drug use. 


\section{Introduction}

Cannabis is the most frequently used illicit drug in the Western world, with up to $32.2 \%$ of adults in the European Union indicating that they have used the drug at least once in their lifetime (EMCDDA, 2011). Data suggest that even though spontaneous cessation of cannabis use occurs, a multitude of cannabis users develop a stable pattern of use, characterized by frequent use of cannabis. In one study, up to $74.2 \%$ of occasional cannabis users went on to develop regular use patterns (Perkonigg et al., 1999).

Cannabis consists of several cannabinoids, with $\Delta 9$-tetrahydrocannabinol (THC) being the psychoactive compound. Acute administration of cannabis in both animals and humans causes impairment in a number of cognitive domains. Administration of a cannabinoid receptor agonist in rats mainly leads to impairments of memory acquisition and working memory (Zanettini et al., 2011) and low doses of cannabis increased motor impulsivity in rodents (Childers \& Breivogel, 1998). Similar findings have been reported in humans as single doses of cannabis impaired memory, attention and motor skills (Gonzalez, 2007; Solowij, 1998). However, several lines of evidence demonstrate that the level of cannabis-induced impairment is inversely related to history of cannabis use. Performance impairments are very prominent in occasional cannabis users (McDonald et al., 2003; Ramaekers et al, 2006a; Crean et al., 2012), but are virtually absent in frequent, heavy users of cannabis (Hart et al., 2010; Ramaekers, Kauert, Theunissen, Toennes, \& Moeller, 2009). The latter has been interpreted as the development of tolerance after repeated cannabis use (Hart et al., 2010; Ramaekers et al., 2009). Studies in frequent cannabis users indicate that single doses of cannabis do not affect a number of cognitive functions such as critical tracking, divided attention or decision making (Ramaekers et al., 2006b; Ramaekers et al., 2009; Ramaekers et al., 2011) when compared to placebo. However, it is not clear whether tolerance to the impairing effects of cannabis extends to every neuropsychological domain. Studies assessing THC effects on impulse control of heavy cannabis users appear to indicate impairment that is not subject to tolerance. This is corroborated by a study in chronic cannabis users who showed impairment on a stop-signal task at THC levels greater than $10 \mathrm{ng} / \mathrm{mL}$, but not when THC blood concentrations were below this level (Ramaekers et al., 2006b; Ramaekers et al., 2009). These findings were also corroborated by Metrik et al. (2012) who demonstrated that single doses of cannabis impaired impulse control in heavy cannabis users and that these users were unable to behaviorally compensate for their intoxications. The current literature 
thus appears to indicate that tolerance to the impairing effect of THC consistently develops in a number of psychomotor domains but may be absent or less prominent in some specific neuropsychological functions requiring impulse control.

Animal research has indicated the likelihood of cross-tolerance between cannabis and cocaine. Pistis et al. (2004) have shown that in adolescent rats which were chronically administered the cannabinoid agonist WIN55212.2, dopamine neurons in the midbrain were less responsive to cocaine than drug-naive rats. This suggests that after subchronic cannabis administration at young age (in rats) subsequent responses to other drugs of abuse, including cocaine, may be altered due to neuronal adaptation. The authors further speculate this adaptation could potentially lead to a decrease in responses to naturally occurring rewarding and motivating stimuli, ultimately leading to increased vulnerability for use of other drugs. In addition, prior exposure to cannabis has been shown to decrease the reinforcing effects and enhance the anxiogenic effects of cocaine (Panlilio, Solinas, Matthews, \& Goldberg, 2007), indicating that THC might potentiate some of the effects of cocaine while negating others. Thus, animal studies indicate the possibility of cross-tolerance to the effects of cocaine after chronic cannabinoid administration. It is not unlikely that humans who are tolerant for cannabis will also develop tolerance for the effects of cocaine. If this is the case, it would pertain to a substantial number of cannabis users as 6 to $20 \%$ of cannabis users in the European Union have used cocaine recently (EMCDDA, 2009).

Cocaine is a stimulating drug, elevating mood, increasing feelings of well-being, energy and alertness and has since long shown to improve performance and reaction speed on measures of information processing, vigilance and divided attention (Burns, 1991, 1992). Most research on the acute effects of cocaine has focused on impulse control, (Fillmore, Rush, \& Hays, 2002; Fillmore et al., 2005, 2006; Garavan et al., 2008) showing that cocaine in general facilitates inhibitory control while decreasing or not affecting reaction times, thereby improving the speed/accuracy trade-off. In line with previous animal research, it is not unlikely that cross-tolerance to the improvement in motor control of cocaine will occur in heavy cannabis users.

The present study was designed to assess whether the impairing effects of THC in heavy cannabis users would present in a wide range of neuropsychological functions or selectively pertain to specific performance domains. A second aim was to assess the acute effects of cocaine on neurocognitive functions of heavy cannabis users. In order to test these aims, a 
number of psychomotor and cognitive tests were included that have previously demonstrated THC induced impairments in occasional but not in heavy users (i.e. divided attention task, critical tracking task, tower of London), as well as a number of impulsivity tasks (Stop-Signal task, matching familiar figures task) for which THC induced impairment has been reported in occasional users but for which presence in heavy users has been disputed (Ramaekers et al., 2006, 2009, 2011). Relative to the latter studies, we increased sample size by a factor 2-3 in order to increase statistical power.

\section{Materials and methods}

Subjects

Sixty-one healthy regular cannabis and cocaine users (48 male, 13 female), participated in the study (demographics: table 1).

\begin{tabular}{llllll}
\hline & Mean & SD & Min & Max & Drug use (yrs) \\
\hline Age (years) & 23,05 & 3,04 & 18,3 & 32,4 & \\
Weight (kg) & 69,34 & 9,98 & 51 & 91 & \\
Cannabis & 1027,43 & 1329,79 & 40 & 6000 & 6,96 \\
Cocaine & 56,54 & 85,58 & 4 & 450 & 3,31 \\
Alcohol & 1063,00 & 2714,01 & 50 & 19200 & 8.54 \\
Ecstasy & 26,81 & 30,74 & 0 & 125 & 4,00 \\
Amphetamines & 44,71 & 167,79 & 1 & 1000 & 2,72 \\
Mushrooms & 7,84 & 14,33 & 0 & 75 & 2,88 \\
LSD & 7,88 & 8,17 & 0 & 20 & 1,64 \\
Other & 17,93 & 37,45 & 0 & 150 & 1,64 \\
\hline
\end{tabular}

Table 1: Mean (SD) age, weight, lifetime drug use (total number of times used) and drug use in years of the 61 subjects enrolled in the study.

Subjects were recruited through advertisements in local newspapers and by word of mouth. Before inclusion, subjects were examined by a study physician, who checked for general health and took blood and urine samples for standard chemistry and haematology. Inclusion criteria were: age 18-40 yrs; regular cannabis use: $\geq 2$ times/week; cocaine use $>5$ times in the previous year; free from psychotropic medication; good physical health; normal 
weight (BMI 18-28) and written informed consent. Exclusion criteria were: dependence on cocaine according to DSM-IV criteria; presence or history of psychiatric or neurological disorder as assessed during a clinical interview; pregnancy or lactating; cardiovascular abnormalities as measured by EKG; hypertension and excessive drinking (>20 units per week) or smoking (>20 cigarettes per day).

This study was part of a larger trial on the association between drug use and impulse control (see Dutch Trial Register, trial number NTR2127) conducted according to the code of ethics on human experimentation established by the declaration of Helsinki (1964) and amended in Seoul (2008) and was approved by the Medical Ethics Committee of the Academic Hospital of Maastricht and Maastricht University. A permit for obtaining, storing and administering cocaine and cannabis was obtained from the Dutch drug enforcement administration. Subjects were paid for their participation in the study.

\section{Design, doses and administration}

Subjects participated in a double-blind, placebo-controlled, 3-way crossover study. Treatments were placebo, $300 \mu \mathrm{g} / \mathrm{kg} \mathrm{THC}$ and $300 \mathrm{mg}$ cocaine $\mathrm{HCl}$. Cannabis was administered through a vaporizer (Volcano) obtained from Storz \& Bickel GmbH \& Co (Tuttlingen, Germany) and was used according to the manual provided by the producer. Cannabis-inhalation took place in a standardized manner (Hazekamp, Ruhaak, Zuurman, Gerven, \& Verpoorte, 2006). The vapor was prepared from batches containing $11 \%$ THC, a standard potency for cannabis sold at Dutch pharmacies for medical/medicinal use. Cannabidiol levels in the cannabis breed were less than 1\%. Preparation of cannabis and cannabis placebo were similar to previous studies from our group (J.G. Ramaekers, Kauert, et al., 2009; Toennes, Ramaekers, Theunissen, Moeller, \& Kauert, 2008). Cocaine $\mathrm{HCl}$ or placebo was administered in an opaque white capsule. Conditions were separated by a minimum wash-out period of 7 days to avoid cross-condition contamination. Order of conditions was balanced over subjects and sessions.

\section{Procedures}

A test-day started with a urine drug screen and a breath alcohol screen; female subjects underwent an additional pregnancy test. If negative (except cannabis) subjects could proceed with breakfast, followed by vital sign measurements and blood samples. 
Immediately hereafter, subjects received a capsule containing either $300 \mathrm{mg}$ cocaine $\mathrm{HCl}$ or placebo orally (T1). Forty-five minutes after capsule administration, subjects inhaled 300 $\mu \mathrm{g} / \mathrm{kg}$ bodyweight cannabis or placebo (T2). Immediately after, vital signs were measured, blood samples were taken and the VAS-questionnaire was administered followed by the test-battery. The latter was conducted between 15-60 minutes following T2. Between T1 and $\mathrm{T} 2$, subjects were allowed to read a book or watch television.

Prior to experimental sessions subjects were familiarized with procedures and tasks. Subjects had to refrain from all drugs of abuse (except cannabis) at least a week before the start of the experiment until study end.

\section{Impulsivity tests}

\section{Matching Familiar Figures task.}

This task is a measure of reflection impulsivity, which is the tendency to reflect on the validity of problem solving under the special condition of several possible alternatives. Thus, subjects who respond quickly often make mistakes, whereas those who reflect on response alternatives are more often correct (Messer, 1976). For a more detailed description of tasks specifics, see van Wel et al. (2012). Two dependent measures, mean latency to first response and total number of errors, were automatically recorded. Two additional dependent variables can be calculated (Perales, Verdejo-Garcia, Moya, Lozano, \& Perez-Garcia, 2009a): an Impulsivity score (I-score) and an Efficiency score (E-score).

\section{The Stop-Signal Task.}

The Stop-Signal task measures motor impulsivity, which is defined as the inability to inhibit an activated or pre-cued response leading to errors of commission (NoGo-errors). The current test has been validated for showing stimulant and sedative drug effects (Ramaekers \& Kuypers, 2006). Dependent variables are the proportion of commission errors on stop trials with a maximum of 48 , omission errors on go-trials and the reaction times on go and stop signal trials (i.e. stop reaction time). Stop reaction time to stop signal trials represents the estimated mean time required to inhibit a response. The method for calculating stop reaction time is taken from the race model of inhibitory control (Logan \& Cowan, 1984). The resulting values for each stop signal delay are averaged to yield a single measure of stop reaction time for the test. 


\section{Psychomotor tests}

\section{Critical Tracking Task.}

The Critical Tracking Task (CTT)(Jex, McDonnell, \& Phatak, 1966) measures the subject's ability to control a displayed error signal in a first-order compensatory tracking task. Error is displayed as a horizontal deviation of a cursor from the midpoint on a horizontal, linear scale. Compensatory joystick movements null the error by returning the cursor to the midpoint. The frequency at which the subject loses the control is the critical frequency or lambda $\left(\lambda_{c}\right)$. The test includes five trials of which the lowest and the highest score are removed; the average of the remaining scores is taken as the final $\lambda_{c}$-score.

\section{Divided Attention Task.}

The Divided Attention Task (DAT) measures the ability to divide attention between two tasks performed simultaneously. Subjects were asked to perform the same tracking task as described above but now at a constant level of difficulty. As a secondary task, the subject was instructed to monitor 24 single digits ( 0 to 9 ) that were presented in the four corners of the computer screen ( 6 digits per corner). These numbers changed asynchronously every 5 seconds. The subjects were instructed to react to the target number ' 2 ' by removing their foot as fast as possible from a pedal switch and return. Inter-target time varied between 5 and 25 seconds with a maximum of 48 targets. Average tracking error, correct responses and mean reaction time (ms) to the target are the performance measures in this secondary subtask.

\section{Tower of London}

The Tower of London (TOL) is a decision-making task that measures executive function and planning (Shallice, 1982). The current version consists of computer-generated images of initial and target-arrangements of three colored balls on three sticks. The subject decides as quickly as possible, whether the end-arrangement can be accomplished in 2, 3, 4, or 5 steps from the initial arrangement by pushing the corresponding coded button. The total number of correct decisions and mean reaction time (in seconds) are the main outcome measures. 


\section{Subjective measures}

\section{Visual Analogue Scales}

Two visual analogue scales $(10 \mathrm{~cm})$ were presented to participants where they had to indicate how 'high' or how 'active' they felt at that moment, compared to the most high or active ever ( 0 represented not high or active at all, 10 extremely high or active).

\section{Pharmacokinetic assessments}

Blood samples to determine drug concentrations were taken at baseline, 50 and 110 minutes after administration of a cocaine or placebo capsule. Blood samples were centrifuged at $2212 \times \mathrm{g}$ and serum was frozen at $-20^{\circ} \mathrm{C}$ until analysis for pharmacokinetic assessments. For cannabinoid determinations serum was used (Serum-Gel Vacuette system of Greiner Bio-One, Alphen a/d Rijn, the Netherlands), cocaine and metabolites were determined in plasma (Glucose FX Vacuette system containing $2.5 \mathrm{mg} / \mathrm{ml}$ sodium fluoride and $2 \mathrm{mg} / \mathrm{ml}$ potassium oxalate). The determination of $\Delta^{9}$-Tetrahydrocannabinol (THC), 11hydroxy-THC (THC-OH), 11-nor-9-carboxy-THC (THC-COOH), cocaine (COC), benzoylecgonine (BZE) and ecgonine methyl ester (EME) in plasma was performed in a specialized forensictoxicological laboratory using validated procedures (Kauert, Iwersen-Bergmann, \& Toennes, 2006; Toennes, Steinmeyer, Maurer, Moeller, \& Kauert, 2005).

\section{Statistics}

All cognitive measures were analyzed with SPSS 18.0 using a GLM univariate analysis of variance (ANOVA) with Drug (3 levels) as within subject factor. If the sphericity assumption was violated, the Greenhouse-Geisser correction was used. In case of significant main drug effects, separate drug-placebo contrasts were conducted. The univariate model tested for main effects of Drug, which were followed by separate drug-placebo contrasts. A sequential Bonferroni procedure was applied to correct for multiple testing. Potential associations between cannabis use history and drug induced performance impairments were assessed by means of Pearson $r$ correlations between drug use history and drug induced change scores from placebo. The $\alpha$-criterion significance level was set to 0.05 . 


\section{Results}

All subjects for whom complete performance data sets were collected entered the GLM analyses. Due to side-effects (nausea) in the cannabis condition, 4 subjects could only partially complete the test battery. In sum, 59 complete datasets were collected for the MFF20, 58 for the TOL and 57 for the SST and CTT. Due to technical failures, only 50 complete datasets could be used in the DAT.

\section{Impulsivity tests}

Mean (SE) performance scores for the MFF20 and SST are shown in figures 1 and 2. THC decreased E-score $\left(F_{1,58}=15.97 ; p<.001\right)$, and increased the number of errors $\left(F_{1,58}=7.90\right.$; $\mathrm{p}=.007)$. Mean latency of first response increased and decreased following THC and cocaine administration respectively, but these changes just failed to reach significance. THC and cocaine did not affect I-scores. On the SST, subjects under the influence of cocaine or cannabis made significantly more commission errors $\left(F_{1,56}=8.51 ; p=.005\right.$ and $F_{1,56}=7.47$, $p=.003$ respectively). Cocaine also decreased reaction time in Go-trials $\left(F_{1,56}=10.19 ; p=.002\right)$. Stop reaction time and number of omission errors were not affected by cannabis and cocaine. There was no correlation between history of cannabis use and performance impairments during cannabis or cocaine on any of the variables of the SST.
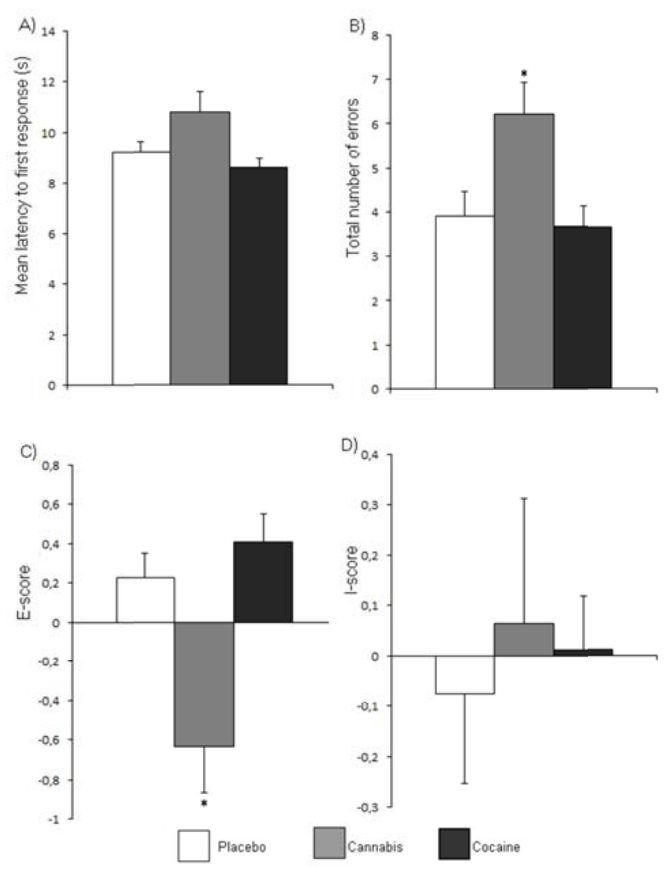

Figure 1: Mean (SE) performance in the Matching Familiar Figures task (MFF20) during cannabis or cocaine intoxication or placebo. Performance parameters include A) mean latency to first response, B) total number of errors, C) E-score) and D) Iscore. *significant drug-placebo contrast with $\mathrm{p}<.05$. 


\section{Psychomotor tests}

In the CTT, performance during cocaine improved relative to placebo as indicated by an increase in $\lambda_{c}\left(F_{1,56}=18.75 ; p<.001\right)$, while THC had the opposite effect $\left(F_{1,56}=4.97 ; p=.03\right)$. Performance on the DAT was significantly affected by cocaine. Cocaine significantly decreased average tracking error $\left(F_{1,49}=28.42 ; p<.001\right)$, reaction time $\left(F_{1,49}=19.22 ; p<.001\right)$ and control losses $\left(F_{1,49}=13.68 ; p=.001\right)$, and increased the correct responses $\left(F_{1,49}=12.68\right.$; $\mathrm{p}=.001$ ) when compared to placebo. THC significantly increased the number of false alarms $\left(F_{1,49}=5.87 ; p=.02\right)$. For mean (SE) scores on the CTT and DAT, see figure 3 . Accuracy of TOL performance was significantly affected by $\operatorname{THC}\left(F_{1,57}=19.95 ; p<.001\right)$, see figure 4 . No effect of either drug on reaction time was found. There was no correlation between history of cannabis use and THC or cocaine intoxication on any of the variables of the CTT, DAT and TOL.
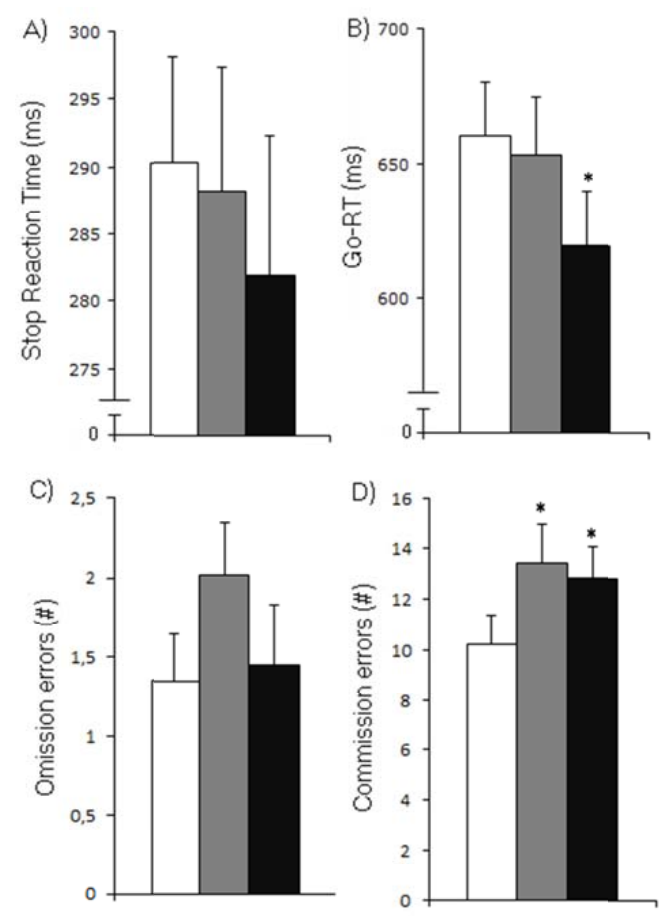

Figure 2: Mean (SE) performance in the Stop Signal task (SST) during cannabis or cocaine intoxication or placebo. Task parameters include A) stop reaction time, B) Go reaction time (in ms), C) number of omission errors and D) number of commission errors. *significant drug-placebo contrast with $p<.05$. 


\section{Subjective measures}

Mean (SE) subjective ratings of 'feeling high' during placebo, THC and cocaine treatment were $1.84(.19), 5.75(.40)$ and $3.53(.37)$ respectively. Mean (SE) ratings of 'feeling active' during placebo, THC and cocaine treatment were 4.44 (.33), 4.47 (.36) and 6.57 (.36) respectively. Cocaine $\left(F_{1,60}=21.5 ; p<.001\right)$ and $\operatorname{THC}\left(F_{1,60}=96.19 ; p<.001\right)$ significantly increased feelings of high relative to placebo. In addition, subjects also felt more active under the influence of cocaine $\left(F_{1,60}=26.68 ; p<.001\right)$, but not during THC intoxication. History of cannabis use and ratings of activity after cocaine administration was positively correlated $(r=.36, p=.004)$. No correlation between ratings of high and history of cannabis use was found in any of the drug conditions.
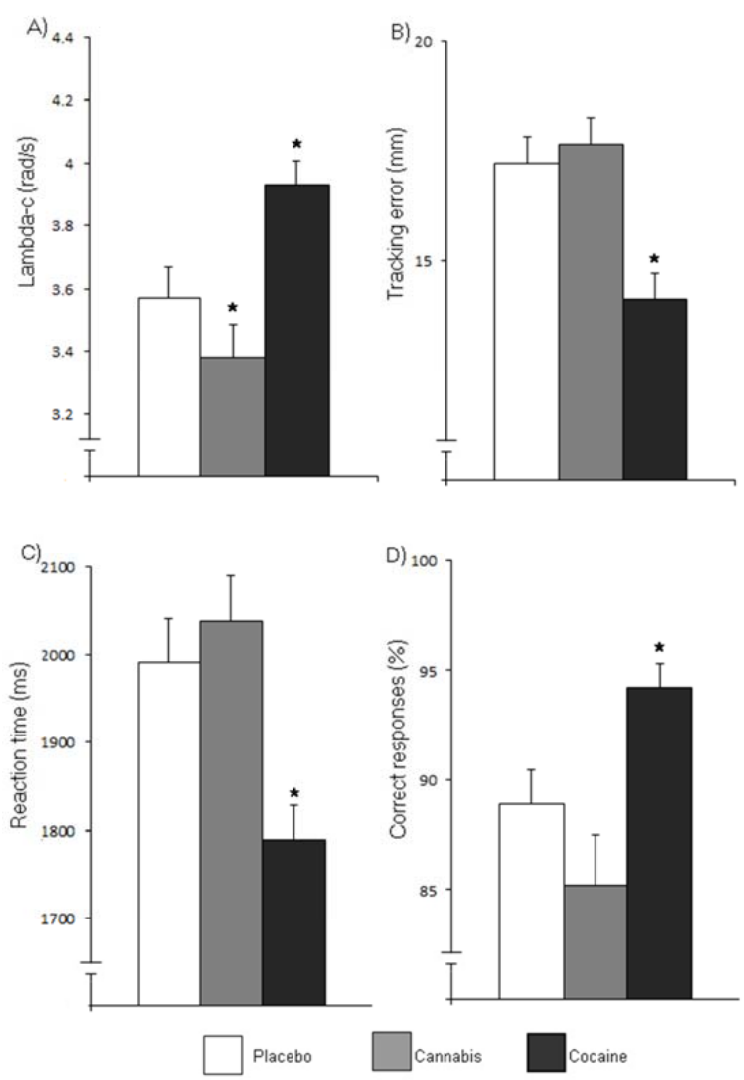

Figure 3: Mean (SE) performance in the CTT (A) and DAT (B-D) during cannabis or cocaine intoxication or placebo. Performance parameters include A) lambda-c (rad/s), B) tracking error (in $\mathrm{mm}$ ), C) reaction time (in ms), D) correct responses. * significant drug-placebo contrast with $\mathrm{p}<.05$. 


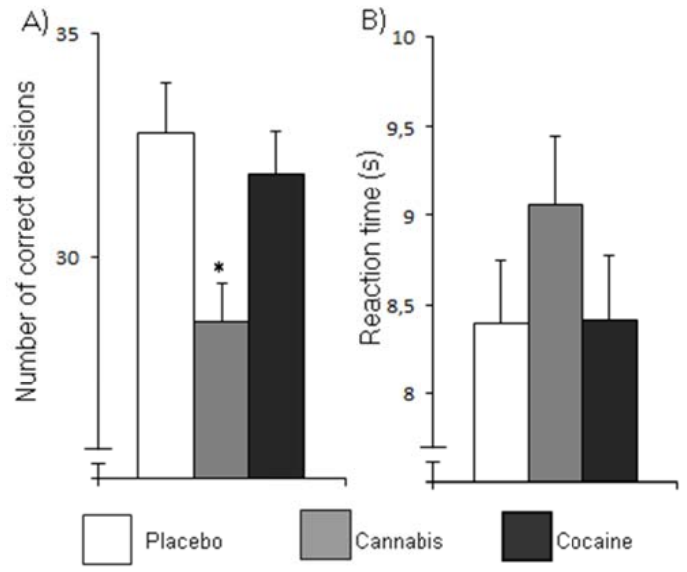

Figure 4: Mean (SE) performance on the Tower of London task (TOL) during cannabis or cocaine intoxication or placebo. Performance parameters include A) number of correct decisions and $\mathrm{B})$ reaction time $(\mathrm{s})$. * significant drug-placebo contrast with $p<.05$.

\section{Pharmacokinetics}

Mean concentration of THC, cocaine and their metabolites are given in Table 2. The cocaine concentrations observed 50 minutes after ingestion are in accordance with previous publications (Van Dyke, Barash, Jatlow, \& Byck, 1976; Wilkinson, Dyke, Jatlow, Barash, \& Byck, 1980) where the large interindividual variability can be explained by variations in the absorption process. Also the cannabinoid concentrations are in the range that has been observed in previous studies with similar THC doses (Toennes et al., 2011, 2008). In some of the conditions a few of the blood samples are missing, e.g. due to some subjects completing the conditions only partially or the inability to draw blood (see table 2).

\section{Discussion and conclusions}

The present study aimed to clarify whether impairing effects of THC in regular cannabis users apply to neuropsychological functions in general or develops selectively in specific performance domains. Furthermore, the study aimed to assess the acute effects of cocaine in regular cannabis users who were expected to be tolerant to the impairing effects of single doses of cannabis.

Results from this study indicate that THC-induced impairments were present in all neuropsychological domains, rather than in some specific domains only. None of the performance tasks were completely unaffected by cannabis inhalation. This contrasts previous results showing that single doses of THC impair critical tracking and divided attention performance in occasional cannabis users but not in heavy, frequent cannabis 


\begin{tabular}{|c|c|c|c|c|}
\hline \multirow[t]{3}{*}{ Drug condition } & \multirow[b]{3}{*}{$\mathrm{N}$} & \multirow{3}{*}{$\begin{array}{l}\text { Time after } \\
\text { cocaine/placebo } \\
\text { Time after THC/placebo }\end{array}$} & \multirow{3}{*}{$\begin{array}{l}-5 \min \\
-45 \min \end{array}$} & \multirow{3}{*}{$50 \mathrm{~min}$} \\
\hline & & & & \\
\hline & & & & \\
\hline \multicolumn{5}{|l|}{ Placebo - Condition } \\
\hline THC & 60 & & $1.4 \pm 3.9$ & $1.8 \pm 3.2$ \\
\hline $\mathrm{THC}-\mathrm{OH}$ & 60 & & $3.9 \pm 0.6$ & $3.2 \pm 0.6$ \\
\hline $\mathrm{THC}-\mathrm{COOH}$ & 60 & & $19.8 \pm 37.5$ & $17.4 \pm 32.7$ \\
\hline \multicolumn{5}{|l|}{ Cannabis - Condition } \\
\hline THC & 56 & & $1.7 \pm 3.4$ & $55.3 \pm 29.5$ \\
\hline $\mathrm{THC}-\mathrm{OH}$ & 56 & & $0.8 \pm 1.7$ & $6.9 \pm 4.8$ \\
\hline $\mathrm{THC}-\mathrm{COOH}$ & 56 & & $24.1 \pm 39.2$ & $41.9 \pm 33.9$ \\
\hline \multicolumn{5}{|l|}{ Cocaine - Condition } \\
\hline THC & 60 & & $1.2 \pm 2.4$ & $1.7 \pm 3.4$ \\
\hline $\mathrm{THC}-\mathrm{OH}$ & 60 & & $0.6 \pm 1.3$ & $0.7 \pm 1.7$ \\
\hline $\mathrm{THC}-\mathrm{COOH}$ & 60 & & $24.0 \pm 55.8$ & $24.6 \pm 54.0$ \\
\hline Cocaine & 57 & & $7.2 \pm 53.7$ & $284 \pm 198$ \\
\hline Benzoylecgonine & 57 & & $40.0 \pm 269.7$ & $509 \pm 226$ \\
\hline Ecgoninemethylester & 57 & & $10.3 \pm 77.2$ & $162 \pm 124$ \\
\hline
\end{tabular}

Table 2: Mean (SD) serum concentrations ( $\mathrm{ng} / \mathrm{mL}$ ) of THC, THC-COOH, THC-OH and mean (SD) plasma concentrations $(\mathrm{ng} / \mathrm{mL}$ ) of Benzoylecgonine, Ecgonine methyl ester, Cocaine prior (TO) and after drug administration

users (Ramaekers et al., 2009; Ramaekers et al., 2011) while using the same psychomotor tests and procedures and comparable THC doses as in the present study. However, the magnitude of THC-induced impairments during critical tracking and divided attention was about a factor 2-3 less in magnitude compared to THC-induced impairments in occasional cannabis users as reported in other studies (Ramaekers et al., 2006; Ramaekers et al., 2009). Although this paper does not compare cannabis effects in occasional and heavy smokers directly, the latter finding seems to support the general notion that heavy users develop some tolerance to the impairing effects of cannabis on psychomotor function, albeit not complete. The sensitivity in the present study to detect mildly impairing effects of THC 
may be related to the large sample size that increased statistical power to significantly detect even the smallest changes in performance.

Data revealed that THC significantly affected performance in the impulsivity tests (MFF20, SST). THC decreased general efficiency in the MFF20 task and increased the number of commission errors in No-Go trials of the SST. Efficiency is a composite measure that takes mean response latency and the number of incorrect responses into account. In the present study, subjects generally responded more slowly in the MFF20 although this change just failed to reach statistical significance. The loss of accuracy however was highly significant during THC intoxication. This may indicate that subjects made more errors even though they took more time to reflect on the presented problems. Subjects also made more commission errors during No-Go trials in the SST after THC intoxication. The increase in errors, however, was unrelated to the general speed of responding in Go and No go trials (i.e. stop reaction time) that remained unaffected during THC when compared to placebo. The same effect was seen in the TOL, where subjects made more errors after THC administration, without affecting reaction time. These data point to the notion that subjects during THC intoxication were unable to alter their first response selection when demanded.

Heavy cannabis users also displayed sensitivity for the stimulant effects of cocaine. Cocaine significantly improved psychomotor performance in the CTT and DAT on a number of performance parameters. It improved tracking performance and choice and speed of responding. Cocaine also produced 'stimulating' effects on the impulsivity tasks. It decreased response time in the MFF20 and in the SST and increased the number of commission errors in the same test. However, cocaine did not affect the composite measures of Impulsivity and Efficiency in the MFF20.

Both THC and cocaine altered the subjective experiences of subjects significantly. After cannabis administration, subjects indicated feeling significantly more high. After cocaine, subjects not only reported feeling high, but they also felt more active. The current results confirm previous findings (Hart et al., 2010; Ramaekers et al., 2009) showing that heavy cannabis users do not develop pharmacological tolerance to the subjective effects of the drug.

The presence of performance impairment during THC intoxication in the present subject sample did not result from inexperience with drug use. All subjects were regular cannabis users who on average reported cannabis drug use in over 1000 occasions. 
Correlational analysis also demonstrated that THC-induced impairments were broadly present in the whole subject sample and were not related to cannabis use history. Likewise, cocaine-induced changes in performance were generally not related to cannabis use history of the present set. Only 1 parameter (subjective rating of activity) was significantly correlated to cannabis use history. This correlation, however, was fairly moderate and not necessarily meaningful.

Both THC and cocaine decreased proficiency of impulse control, but in apparently opposing ways. Results indicate that subjects during THC and cocaine intoxication were unable to inhibit a response when requested and that such errors occurred because subjects generally responded too fast during the cocaine condition and too slow during THC intoxication. These differential effects of THC and cocaine on impulse control may be related to differential pharmacological actions of both drugs in the limbic circuit associated with impulse control and addiction. The circuit has been broadly described to consist of a reflective system located in the medial prefrontal cortex and reactive system located in the striatum. The reactive system generates impulses and reward following dopaminergic stimulation of the nucleus accumbens, whereas the reflective systems exacerbates glutamatergic control over impulses generated in the striatum (Bechara, 2005; Faure, Richard, \& Berridge, 2010; Kalivas \& Volkow, 2005). Cocaine is known to increase dopamine levels in parts of the striatum that innervate limbic and motor circuits (Breiter et al., 1997). This may account for the finding that subjects respond faster and more impulsive during cocaine. The effects of single doses of THC are not as clear, indicating either an increase (Bossong et al., 2009) or decrease of dopamine levels in the striatum (Barkus et al., 2011; Kuepper et al., 2013). Recent studies indicate that THC decreases dopamine levels throughout the brain after chronic exposure (Marco Pistis et al., 2004; Urban, Slifstein, Thompson, et al., 2012). Subjects in the present study were all heavy users who had been using for an average of 7 years. The reduction in proficiency of impulse control during THCintoxication is thus less likely to result from overactivation of the 'reactive' part of the 'reward' circuit and more likely to result from a deactivation of the 'reflective' part of the 'reward' system. More research is needed to confirm differential activations of the frontal cortex and limbic system after THC and cocaine administration, particularly in the context of repeated drug use or addiction. Reduction in proficiency of impulse control during cannabis 
and cocaine intoxication stimulates repeated drug use and may as such even play an important role in the neuropathology and development of drug addiction.

In sum, the present study demonstrated that heavy cannabis users showed impairment in a broad range of neuropsychological domains during THC intoxication. Impairments observed in psychomotor tasks however appeared smaller in magnitude as compared to those previously reported in occasional cannabis users. Administration of THC also affected performance of heavy users in tests of impulse control. Heavy cannabis users were sensitive to the stimulatory effects of cocaine. Cocaine increased psychomotor function and decreased response time on an impulsivity task at the cost of making more errors. It was speculated that the reduction in proficiency of impulse control observed during THC and cocaine intoxication may put drug users at risk of repeated drug use and addiction. 
361 


\section{CHAPTER 3}

TRAIT IMPULSIVITY CONTRIBUTES TO PSYCHEDELIC EXPERIENCE OF CANNABIS AND COCAINE USE use. 


\section{Summary}

Trait impulsivity has been linked to addiction in humans since impulsive individuals appear more susceptible to repeated drug use and relapse. It has been suggested that drug users with high trait impulsivity levels are more sensitive to the subjective effects of drugs of abuse. The present study assessed whether the subjective response to drugs differs between drug users that have normal or high levels of trait impulsivity. Regular cannabis and cocaine users ( $\mathrm{N}=122$ ) participated in a double-blind, placebo controlled, 3-way crossover study. Subjects received doses of cocaine $\mathrm{HCl}$, cannabis and placebo and completed a number of subjective questionnaires measuring mood, dissociative state, and psychedelic experience by means of the profile of mood states (POMS), the Clinician Administered Dissociative States Scale (CADSS), and the Bowdle visual analog scale (B-VAS). Trait impulsivity was assessed at baseline with the Barratt Impulsiveness Scale (BIS-11). Overall, cannabis intoxication increased ratings of dissociation and psychedelic state, as well as feelings of fatigue, confusion, depression and anxiety, and decreased feelings of arousal, positive mood, vigor, friendliness and elation. Cocaine significantly increased ratings of dissociation, psychedelic state and feelings of vigor, friendliness, elation, positive mood, anxiety and arousal, while decreasing fatigue. Psychedelic experiences following cannabis use were most intense in high impulsivity subjects. Feelings of dissociation following cocaine were also more intense in high impulsivity subjects. It is concluded that trait impulsivity contributes to subjective experiences of drug use and may increase the risk of developing drug use problems. 


\section{Introduction}

Impulsivity refers to actions that are poorly conceived, risky or premature and that may result in unfavorable outcomes (Evenden, 1999; Stanford et al., 2009). Pathological forms of impulsivity may contribute to the etiology of mental disorders such as addiction (de Wit, 2008). A range of animal studies have shown that an association exists between trait impulsivity and addiction (Belin, Mar, Dalley, Robbins, \& Everitt, 2008; Economidou, Pelloux, Robbins, Dalley, \& Everitt, 2009). Rats with higher trait impulsivity were more likely to score higher on measures of addiction, e.g. self-administration rates, following drug intake than animals with lower impulsivity scores. The same has been shown in humans. Persons exhibiting high levels of trait impulsivity are more susceptible to substance abuse and display increased relapse rates after drug abstinence (Pattij \& De Vries, 2013). Also, among drug addicts, comorbidity with other mental disorders associated with pathological impulsivity such as ADHD (Wilens \& Morrison, 2011) and bipolar disorder (Pettinati, Brien, \& Dundon, 2013) is very high.

Cannabis and cocaine are respectively the first and second most used drugs in the European Union (EMCDDA, 2012). Although mood and psychedelic effects after cannabis administration have been extensively investigated (Battistella et al., 2013; D'Souza et al., 2008; Henquet et al., 2010; Lex, Mendelson, Bavli, Harvey, \& Mello, 1984; Ramaekers et al., 2006; Toennes, Ramaekers, Theunissen, Moeller, \& Kauert, 2008), there is a relative paucity in subjective data after cocaine administration. It has been shown that cocaine administration has a stimulating effect on mood (Penetar et al., 2006; Rush et al., 1999; Walsh et al., 2010), but these studies mainly measured general drug effects such as 'feeling good' and 'wanting to take again'. Also, although epidemiological evidence has associated extensive cocaine use with psychotic disorders (Morton, 1999; Roncero et al., 2012) and medication with a similar mechanism of action as cocaine, such as dexamphetamine and methylphenidate, has been shown to increase the likelihood of psychotic symptoms (Curran et al., 2004), no studies so far have investigated the acute effects of cocaine on measures of dissociation or psychedelic experiences.

Likewise, only few studies have assessed the role of trait impulsivity on the subjective experience of drug intoxication. High trait impulsivity was significantly associated with greater arousal and euphoria following administration of d-amphetamine (Kirkpatrick et al., 2013; White, Lott, \& de Wit, 2006). In the present study we examined whether the level of 
trait impulsivity (normal or high) influences the subjective response to acute administration of cannabis or cocaine. We hypothesized that administration of cannabis and cocaine to high impulsive subjects would evoke more intense subjective effects as compared to normal impulsive subjects. Increased sensitivity to subjective effects of cannabis and cocaine may be particularly relevant in the realm of drug addiction and contribute to an individual's desire to seek drugs. Subjective measures of mood, dissociative state and psychedelic experience scales were used to assess psychological states during drug intoxication.

\section{Materials and methods}

\section{Subjects}

122 healthy regular cannabis and cocaine users (96 male, 26 female; mean (SD) age 22.84 (3.69), participated in two centers, Maastricht and Nijmegen. In Maastricht, subjects used cannabis on average 51.20 times in the past 3 months, while cocaine was used 4.53 times in the past 3 months. In Nijmegen, subjects used cannabis and cocaine 39 and 2.74 times respectively in the past 3 months. Overall, cannabis was used 44.78 times in the past 3 months and cocaine was used 3.68 times in the past 3 months. Participants also indicated having used other substances in their lives, i.e. $88 \%$ used XTC, $73 \%$ used amphetamines, $61 \%$ used mushrooms, 20\% used LSD and 59\% used other drugs including but not limited to laughing gas, MDMA crystals, DMT and ketamine.

Subjects were recruited through advertisements in local newspapers and by word of mouth. Before inclusion, subjects were examined by a physician. They were checked for general health and blood and urine samples were taken for standard chemistry and haematology. Inclusion criteria were: written informed consent; age 18-40 yrs; regular cannabis use: $\geq 2$ times/week; have used cocaine at least 5 times in the past year free from psychotropic medication; good physical health; normal weight (BMI 18-28). Exclusion criteria were: cocaine dependence according to DSM-IV criteria; presence or history of psychiatric or neurological disorder as assessed during a clinical interview; pregnancy or lactating; cardiovascular abnormalities; excessive drinking or smoking, and hypertension.

This study was part of a larger trial on the association between drug use and impulse control (see Dutch Trial Register, trial number NTR2127) conducted according to the code of ethics on human experimentation established by the declaration of Helsinki (1964) and amended in Seoul (2008) and was approved by the Medical Ethics Committee of the 
Academic Hospital of Maastricht and Maastricht University. A permit for obtaining, storing and administering cocaine and cannabis was obtained from the Dutch drug enforcement administration. Subjects were paid for their participation in the study.

\section{Design, doses and administration}

Subjects participated in a double-blind, placebo-controlled, double-dummy, 3-way crossover study. Treatments were placebo, $450 \mu \mathrm{g} / \mathrm{kg}$ THC (cannabis plant material, divided in two doses of 300 and $150 \mu \mathrm{g} / \mathrm{kg}$ ) as well as $300 \mathrm{mg}$ cocaine $\mathrm{HCl}$. In the Nijmegen center, a booster dose of $150 \mathrm{mg}$ cocaine was given to a subset of subjects. Cannabis was administered using a vaporizer (Volcano) obtained from Storz \& Bickel GmbH \& Co (Tuttlingen, Germany) and was used according to the manual provided by the producer. Cannabis-inhalation took place in a standardized manner (Hazekamp et al., 2006). The vapor was prepared from batches varying between $11-12 \%$ THC, a standard potency for cannabis sold at Dutch pharmacies for medical/medicinal use. The varying potency was adjusted for in the weighing of the plant material. Cocaine $\mathrm{HCl}$ or placebo was administered in an opaque white capsule. Conditions were separated by a minimum wash-out period of 7 days to avoid cross-condition contamination. Order of conditions was balanced over subjects and sessions.

\section{Procedures}

Prior to experimental sessions, subjects were familiarized with procedures on a separate training day. Subjects had to refrain from all drugs of abuse (except cannabis) at least a week before the start of the experiment until study end.

A test-day started with a urine drug screen to assess the presence of benzodiazepines, opiates, cocaine, marijuana, MDMA and (meth)amphetamine and a breath alcohol screen; female subjects underwent an additional pregnancy test. If negative for substances (except cannabis) or pregnancy, subjects could proceed with breakfast. This was followed by vital sign measurements and blood samples. Immediately hereafter, subjects received a capsule containing either $300 \mathrm{mg}$ cocaine $\mathrm{HCl}$ or placebo orally (T0). Forty-five minutes after T0, subjects inhaled $300 \mu \mathrm{g} / \mathrm{kg}$ cannabis or placebo (T1). Immediately after T1, vital signs were measured, blood samples were taken and the Profile of Mood States questionnaire (POMS) was administered. One hour after T1, a second dose THC $150 \mu \mathrm{g} / \mathrm{kg}$ or placebo was given (T2). In the Nijmegen center a subset $(\mathrm{N}=47)$ of subjects received a second 
dose of cocaine $150 \mathrm{mg} \mathrm{HCl}$ just prior to administration of B-VAS. The aim of that booster dose was to prolong cocaine concentrations during task performance that were conducted after completion of the questionnaires and that will be reported elsewhere. As such the booster dose was not expected to affect B-VAS ratings because both were administered concurrently but it might have affected CADSS ratings in this subsample since these were taken 2 hours post the booster dose. At this time, blood samples were also taken. See for a schematic representation of the testing day figure 1.

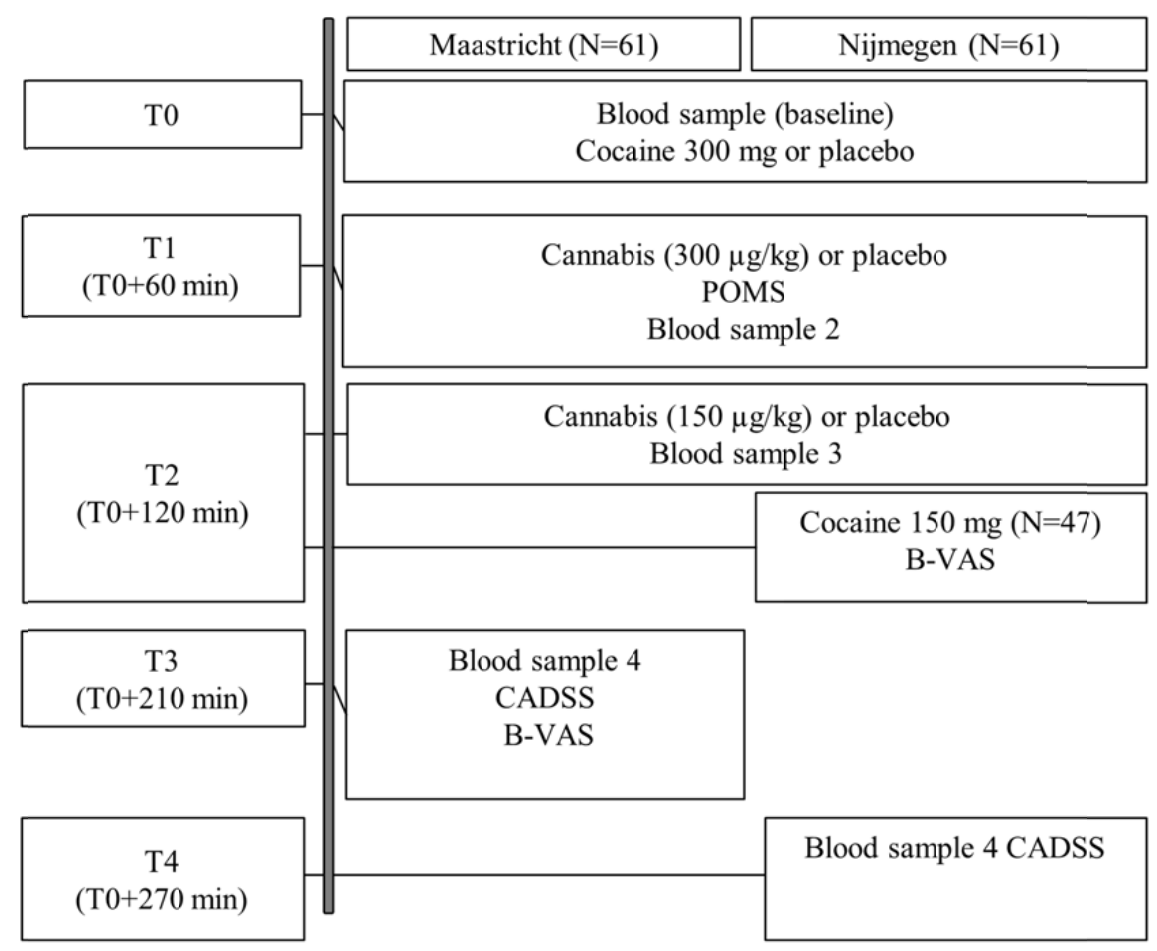

Figure 1: schematic representation of a testing day. On the left the time is indicated from T0 to T4. On the right, the procedure per center is presented.

\section{Questionnaires}

\section{Barratt Impulsiveness Scale}

The Barratt Impulsiveness Scale version 11 (BIS-11) is a 30-item self-report instrument designed to assess trait impulsiveness (Stanford et al., 2009). The BIS-11 has 3 subscales: 1) motor impulsivity, or acting without thinking, 2) attentional impulsiveness, inability to focus attention, and 3) non-planning, or not thinking carefully. Taken together these subscales 
form a total impulsivity score. A total score of 72 or above is taken as an indication of high impulsiveness, scores between 52 and 71 are indicative of normal impulsiveness and scores lower than 52 are representative of an individual that is unusually over-controlled or who has not honestly completed the questionnaire (Stanford et al., 2009). The BIS-11 was administered during the training session.

\section{Bowdle Visual Analogue Scales}

To assess the psychedelic effects of cannabis and cocaine, a 13-item Visual Analogue Scale (B-VAS) first described in Bowdle et al (1998) was employed. From the B-VAS, composite scores of 'internal perception' (5 items) and 'external perception' (6 items) were calculated using a log-transformation as described in (Zuurman et al., 2008). In addition, all 13 items were analyzed separately.

\section{Profile of Mood States}

The Profile of Mood States (POMS) is a self-assessment mood questionnaire with 72 items, rated on a 5-point Likert scale, with 0 being 'not at all' to 4 'extremely'. Subjects had to indicate to what extent these items were representative of their mood at that moment in time. Eight mood states are classified and quantified by calculating the sum score of associated items for each mood state, i.e., anxiety (9 items), depression (15 items), anger (12 items), vigor (8 items), fatigue (7 items), confusion (7 items), friendliness (8 items) and elation (6 items). Two composite scales were derived; arousal ((anxiety+vigor)-(fatigue+confusion)) and positive mood (elation-depression) (Harriet de Wit, Enggasser, \& Richards, 2002).

\section{Clinician Administered Dissociative States Scale}

The Clinician Administered Dissociative States Scale (CADSS) (Bremner et al., 1998) comprises 19 subjective items, ranging from 0 'not at all' to 4 'extremely. It is divided into 3 components: 1) depersonalization, 2) derealization and 3) amnesia. Summed together, these subscales form a total dissociative score. The CADSS is specifically designed to be a standardized measure of present-state dissociative symptomatology. 


\section{Pharmacokinetic assessments}

Blood samples to assess drug concentrations were taken directly after T2 and 2 hours after T2. Blood samples were centrifuged at $3500 \mathrm{rpm}$ and serum was frozen at $-20^{\circ} \mathrm{C}$ until analysis for pharmacokinetic assessments. For cannabinoid determinations serum was used (Serum-Gel Vacuette system of Greiner Bio-One, Alphen a/d Rijn), cocaine and metabolites were determined in plasma (Glucose FX Vacuette system containing $2.5 \mathrm{mg} / \mathrm{ml}$ sodium fluoride and $2 \mathrm{mg} / \mathrm{ml}$ potassium oxalate). The determination of $\Delta^{9}$-Tetrahydrocannabinol (THC), 11-hydroxy-THC (THC-OH), 11-nor-9-carboxy-THC (THC-COOH), cocaine (COC), benzoylecgonine (BZE) and ecgonine methyl ester (EME) in plasma was performed in a specialized forensic-toxicological laboratory using validated procedures (Toennes et al., 2008, 2005).

\section{Statistics}

All cognitive measures were analyzed with SPSS 18.0. First general linear model (GLM) analyses were conducted using repeated measures analysis of variance (ANOVA) with Drug ( 3 levels) as within subject factor and Center (Maastricht vs Nijmegen) as between subjects factor in order to check for treatment differences between the two centers. In case of a significant difference between centers, correlation analyses between those variables and THC serum concentration and cannabis use history were conducted. Further GLM ANOVAs were done with Drug (3 levels) as within subject factor, trait Impulsivity (2 levels: high vs. normal) as between subjects factor and Drug $x$ Impulsivity as the interaction term. If the sphericity assumption was violated, the Greenhouse-Geisser correction was used. In case of significant overall effect of Drug or Drug $\mathrm{x}$ Impulsivity separate drug-placebo contrasts were conducted to establish the effects of THC and cocaine (cannabis vs placebo and cocaine vs placebo) and their interaction with impulsivity level (cannabis vs placebo * impulsivity score, cocaine vs placebo * impulsivity score). The alpha criterion level of significance was set at $p=0.05$.

\section{Results}

All subjects for whom complete performance data sets were collected entered the GLM analyses. Complete datasets of the POMS were collected in 121 subjects, the CADSS was complete in 115 subjects and datasets of the Bowdle-VAS were complete in 96 subjects. 
Missing data was due to incomplete data (1 case in the POMS, 5 cases in the CADSS) or adverse effects (nausea) in the cannabis condition (2 cases in the CADSS). The B-VAS were completed in a subset due to late inclusion of the questionnaire in the study protocol.

Impulsivity score (BIS-11)

Forty-nine subjects had a score higher than 72 and scored as 'high impulsive' on the BIS-11, 71 scored as 'normal impulsive' with a score between 52-71. Two subjects did not fill out the BIS and could not be contacted to correct this. These 2 groups were thus included in the statistical model as a between-subjects parameter.

\section{Bowdle VAS}

Mean (SE) B-VAS-scores in every treatment condition and a summary of significant changes induced by THC, cocaine and their interaction with Impulsivity as indicated by GLM drugplacebo contrast analyses are given in Table 1.

Overall, all separate items of the B-VAS were affected by Drug $(p<.015)$. Following drug-placebo contrasts it was shown that both cannabis and cocaine increased the scores for internal and external perception significantly $(p<.001)$, with subjects during cannabis intoxication scoring higher than after cocaine administration.

Drug-placebo contrasts showed that cannabis increased subjective rating on all B-VAS items, whereas cocaine increased ratings in about half of the items. Two items reached significance for the Cocaine $x$ Impulsivity interaction, namely item 5 and 9. High impulsive subjects under the influence of THC had increased difficulty in controlling their thoughts, as well as having the idea that events, objects or other people had particular meaning that was specific for them. Comparison of datasets from the centers revealed that subjective feelings of high following cannabis and cocaine were generally higher in subjects recruited by the Nijmegen center as compared to the Maastricht center. There was no significant correlation between blood concentration of THC or Cannabis use history and the item High. No other BVAS scales were affected by the factor Center. 


\section{POMS}

Mean POMS-scores in every treatment condition and a summary of significant changes induced by THC, cocaine and their interaction with Impulsivity as indicated by GLM drugplacebo contrast analyses are given in Table 2.

Overall analyses revealed an effect of Drug on arousal, anxiety, positive mood, confusion, fatigue, elation, vigor, depression and friendliness $(p<.001)$. Separate drugplacebo contrasts revealed that subjects under the influence of THC showed increased levels of fatigue, confusion, depression and anxiety, while arousal, positive mood, vigor, friendliness and elation were significantly decreased. Under the influence of cocaine, subjects rated themselves as significantly more vigorous, friendly, elated, anxious, aroused and as having a higher positive mood. Subjects were also significantly less fatigued after cocaine administration.

The factor Impulsivity did not affect POMS rating, but the interaction between Drug $\mathrm{x}$ Impulsivity reached significance for arousal and approached significance for vigor. However, separate drug-placebo contrasts showed no significant effects.

Comparison of datasets from the centers revealed that subjective feelings differed on almost all subscales of the POMS. Under the influence of cannabis, subjects in Nijmegen had a significantly decreased score on the subscales elation, vigor, friendliness and arousal. Fatigue, anxiety and confusion were significantly increased in the Nijmegen center during cannabis intoxication when compared to the Maastricht center. There were no group effects for cocaine. Correlation analyses did not show a relationship between THC concentrations and the subscales of the POMS on which a group effect was shown. However, there was a correlation between cannabis use history and almost every subscale of the POMS. Cannabis use history was inversely correlated with anxiety, depression, anger, fatigue and confusion (range $r=-.36$ to $r=-.21, p<.02$ ). There was a positive correlation between cannabis use history and friendliness, arousal and positive mood (range $r=.27$ to $r=.21, p<.03$ ). 


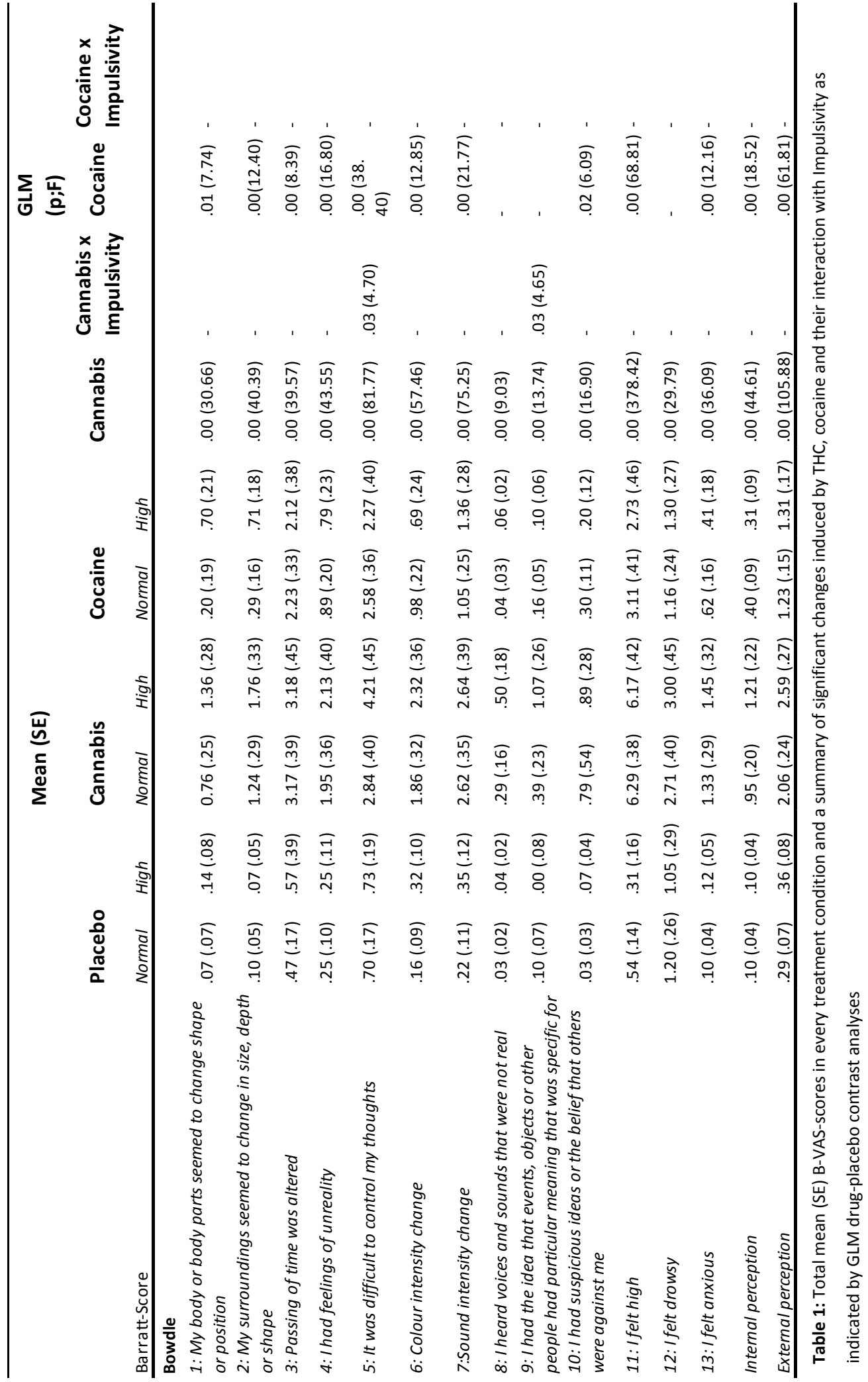




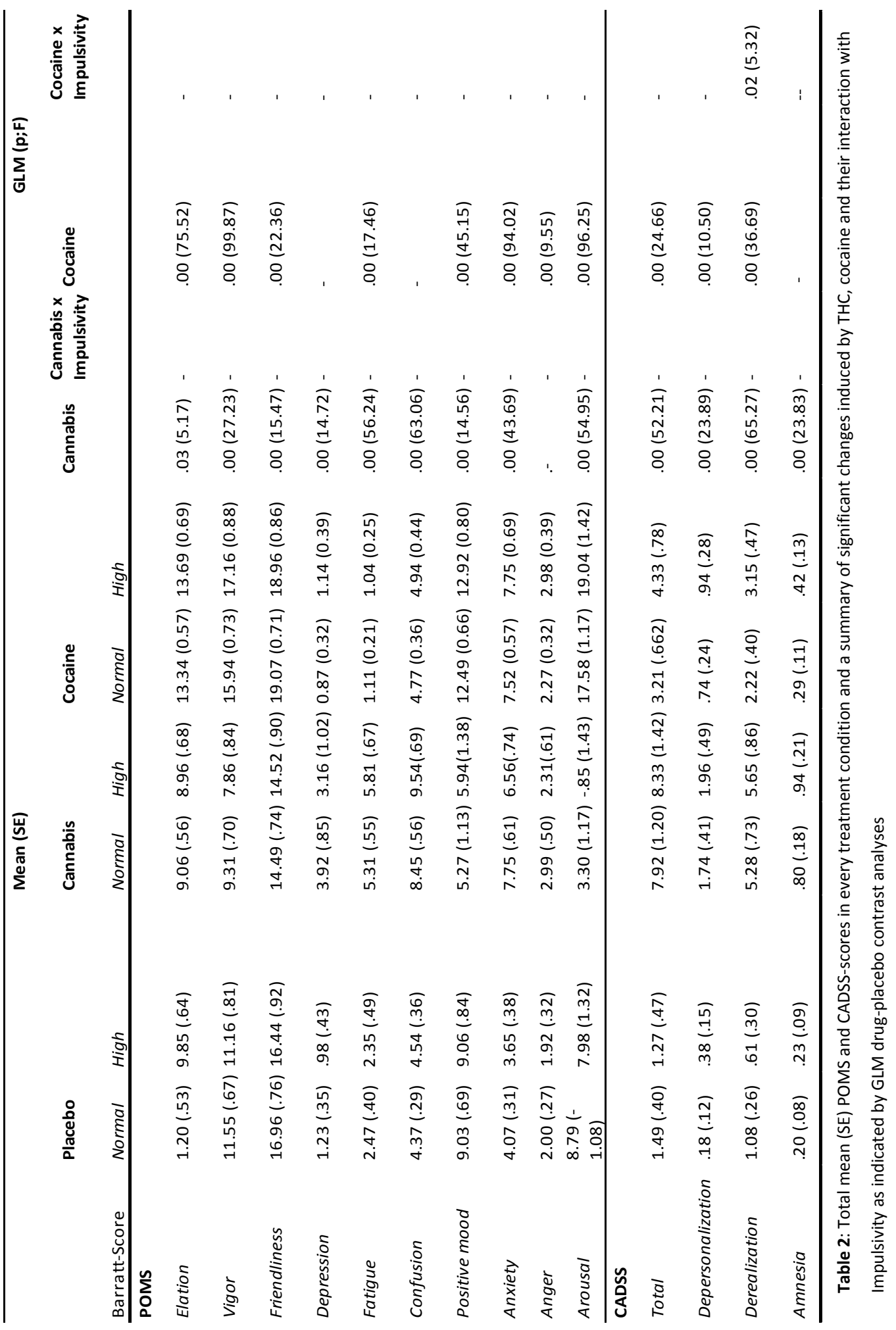




\section{CADSS}

Mean (SE) CADSS-scores in every treatment condition and a summary of significant changes induced by THC, cocaine and their interaction with Impulsivity as indicated by GLM drugplacebo contrast analyses are given in Table 2.

Overall, subjective ratings of depersonalization, derealization and amnesia were affected by the factor Drug. Separate drug-placebo contrasts revealed that both THC and cocaine significantly increased subjective ratings of depersonalization and derealization. In addition, THC also significantly increased subjective ratings of amnesia. THC effects on CADSS-scores did not differ between both Impulsivity groups. Cocaine effects on derealization however were more prominent in the group with high trait impulsivity $\left(F_{1,108}=\right.$ $5.32, \mathrm{p}<.02)$. Subjective ratings during drug treatments did not differ between the two study centers.

\section{Pharmacokinetic assessments}

Mean (SD) concentrations of THC, cocaine and their metabolites per study center are given in Table 3. Mean concentrations of THC were significantly $\left(F_{1,91}=18.16, p<.001\right)$ higher in subjects that participated in the Nijmegen center as compared to the Maastricht center.

\begin{tabular}{llllllll}
\hline Study center Time point THC $[\mu \mathrm{g} / \mathrm{L}]$ & $\begin{array}{l}\mathrm{THC}-\mathrm{OH} \\
{[\mu \mathrm{g} / \mathrm{L}]}\end{array}$ & $\begin{array}{l}\mathrm{THC}-\mathrm{COOH} \\
{[\mu \mathrm{L} / \mathrm{L}]}\end{array}$ & $\begin{array}{l}\text { Cocaine } \\
{[\mathrm{mg} / \mathrm{L}]}\end{array}$ & $\begin{array}{l}\text { Benzoyl- } \\
\text { ecgonine } \\
{[\mathrm{mg} / \mathrm{L}]}\end{array}$ & $\begin{array}{l}\text { Ecgonine- } \\
\text { methyl- } \\
\text { ester } \\
{[\mathrm{mg} / \mathrm{L}]}\end{array}$ \\
\hline Maastricht & $T 0$ & $1.62(3.38)$ & $0.75(1.64)$ & $23.72(38.90)$ & $0.00(0.00)$ & $0.00(0.02)$ & $0.00(0.00)$ \\
& $T 1$ & $55.34(29.20)$ & $6.90(4.76)$ & $41.31(33.89)$ & $0.27(0.20)$ & $0.50(0.24)$ & $0.16(0.12)$ \\
& $T 2$ & $37.62(19.63)$ & $5.90(3.50)$ & $43.41(32.38)$ & $0.32(0.19)$ & $1.02(0.37)$ & $0.25(0.11)$ \\
& $T 3$ & $8.41(5.02)$ & $3.22(1.89)$ & $36.36(30.90)$ & $0.16(0.10)$ & $1.27(0.53)$ & $0.24(0.10)$ \\
Nijmegen & $T 0$ & $1.52(4.75)$ & $0.51(1.78)$ & $18.38(36.66)$ & $0.00(0.02)$ & $0.01(0.04)$ & $0.00(0.01)$ \\
& $T 1$ & $90.49(79.19)$ & $6.81(3.92)$ & $36.31(33.01)$ & $0.23(0.19)$ & $0.48(0.30)$ & $0.12(0.08)$ \\
& $T 2$ & $54.07(42.54)$ & $5.70(3.54)$ & $41.17(39.49)$ & $0.31(0.17)$ & $1.21(0.34)$ & $0.26(0.09)$ \\
Average & $T 4$ & $4.55(2.95)$ & $2.04(1.23)$ & $30.21(27.65)$ & $0.18(0.09)$ & $1.80(0.44)$ & $0.33(0.12)$ \\
& $T 0$ & $1.57(4.14)$ & $0.62(1.72)$ & $20.91(37.68)$ & $0.00(0.02)$ & $0.00(0.03)$ & $0.00(0.01)$ \\
& $T 1$ & $73.81(63.09)$ & $6.86(4.32)$ & $38.69(33.38)$ & $0.25(0.19)$ & $0.49(0.27)$ & $0.14(0.11)$ \\
& $T 2$ & $46.00(34.19)$ & $5.79(3.51)$ & $42.27(36.03)$ & $0.31(0.18)$ & $1.12(0.37)$ & $0.26(0.10)$ \\
& T3/T4 & $6.46(4.52)$ & $2.63(1.69)$ & $33.25(29.33)$ & $0.17(0.09)$ & $1.54(0.55)$ & $0.29(0.12)$ \\
\hline
\end{tabular}

Table 3: Mean (SD) values of THC, cocaine and their metabolites separately and averaged over study center 


\section{Discussion}

The present study aimed to assess whether the level of trait impulsivity influences the subjective response to acute administration of cannabis or cocaine as determined by questionnaires measuring mood, dissociative states and psychedelic experience.

Results indicate that administration of cannabis and cocaine significantly altered subjective ratings on nearly all subscales of all questionnaires. Cocaine significantly increased ratings of dissociation, psychedelic state and feelings of vigor, friendliness, elation, positive mood, anxiety and arousal, while decreasing fatigue. The effects on mood are in accordance with other studies also showing mood improvement following cocaine (Penetar et al., 2006; Rush et al., 1999; Walsh et al., 2010). Increased ratings of dissociation and psychedelic state following cocaine appear in line with studies reporting similar findings after administration of other stimulant drugs such as dexamphetamine and methylphenidate (Curran et al., 2004). Cocaine use has been implied in the occurrence of psychotic disorders, albeit mostly with long-term cocaine 'crack' users (Morton, 1999; Roncero et al., 2012). Although none of the present subjects developed a psychosis, the present results do suggest that cocaine can cause dissociative symptoms (e.g. depersonalization, derealization, psychedelic experience) even in non-addicted cocaine users.

Cannabis intoxication increased ratings of dissociation and psychedelic state, as well as feelings of fatigue, confusion, depression and anxiety, and decreased feelings of arousal, positive mood, vigor, friendliness and elation. Previous studies have also reported that THC increased subjective feelings of high, fatigue (Ballard, Bedi, \& de Wit, 2012; Ballard, Gallo, \& de Wit, 2013; Battistella et al., 2013; D'Souza et al., 2008, 2012; Henquet et al., 2010; Kaufmann et al., 2009; Kirk, Doty, \& De Wit, 1998; Lex et al., 1984; Ramaekers et al., 2006; Ramaekers et al., 2006; Toennes et al., 2008; Zuurman et al., 2008), confusion and anxiety (Battistella et al., 2013; D’Souza et al., 2008; Lex et al., 1984). Likewise, previous research has shown that THC can induce psychedelic or dissociative states as assessed with a CADDS questionnaire, Hallucinogen Rating Scale or a Psychotomimetic States Inventory (Ballard et al., 2012; D'Souza et al., 2008, 2012; Stokes et al., 2009; Zuurman et al., 2008). The latter scale particularly identified perceptual distortion, cognitive disorganization and mania as major psychotomimetic symptoms following acute THC intoxication (Stokes et al., 2009). In general the scope of subjective experiences reported by the subjects in the present study appears in line with previous reports on the use of cannabis. 
Trait impulsivity of subjects was classified using the Barratt Impulsiveness Scale. Impulsivity levels of about $60 \%$ of the subjects were classified as normal whereas the remaining $40 \%$ displayed high levels of impulsivity. GLM analyses revealed that the subgroup of high impulsive subjects were more sensitive to some of the psychedelic and dissociative effects produced by cocaine and THC. Feelings of derealization following cocaine administration were more prominent in high impulsive subjects as compared to subject with normal levels of impulsiveness. During THC intoxication, high impulsive subjects displayed stronger psychedelic experiences than subjects will normal impulsivity levels. They reported more difficulties controlling thoughts and were more likely to attribute personal meaning to events, objects and other people. Drug induced states of mood, as assessed with the POMS rating scale, were not affected by the level of trait impulsivity. These data suggest that impulsivity level partially affects or predicts the subjective experience of subjects during cannabis and cocaine intoxication, and that subjective drug experience in high impulsive subjects may be more intense.

It has been suggested that personality traits may in part determine risk of developing substance use problems (Koob, Sanna, \& Bloom, 1998; Kreek, Nielsen, Butelman, \& LaForge, 2005). Preclinical work has demonstrated that impulsive trait predicts susceptibility to drug seeking and relapse (Everitt et al., 2008). Imaging work in drug users revealed enhanced mesolimbic activity and dopamine release in response to pharmacological reinforcers in subjects with high impulsivity levels and suggested that a pattern of exaggerated dopamine response could develop into a pathological state (Buckholtz et al., 2010). Drugs administered in the present study are also known to affect dopamine release in the brain. Cocaine has been shown to increase tonic dopamine levels in the mesolimbic circuit (Di Chiara \& Imperato, 1988). The effects of cannabis on dopamine release are indirect and more complex. There is preclinical evidence that cannabis increases dopamine in the mesolimbic circuit during occasional, infrequent use but decreases dopamine levels following repeated, chronic use (Marco Pistis et al., 2002; Verrico et al., 2003). Subjects participating in the present study were all very experienced and regular cannabis users. Findings from preclinical studies thus suggest that subjective effects of cocaine in the present study would result from a hyperdopaminergic state and subjective experience of cannabis from a hypodopaminergic state. Subjective experiences may have been more prominent in subjects displaying very low or very high levels of tonic dopamine. Is has been suggested that the 
relation between impulse control and dopamine follows a U-shaped curve (Kreek et al., 2005; Weinshenker \& Schroeder, 2007). Individuals with relatively low or high levels of tonic dopamine may be more vulnerable to loss of impulse control whereas individuals with intermediate levels of tonic dopamine may exert optimal control over their impulses. It is conceivable that both low and high levels of tonic dopamine underlie Barratt's classification of high trait impulsivity. In such a scenario, cannabis would exacerbate subjective experiences in high impulsive subjects with low tonic dopamine levels whereas cocaine would primarily exacerbate subjective experience in high impulsive subjects with high tonic dopamine levels.

The present study was conducted in two study centers that followed the same study protocols. We conducted GLM analysis to evaluate whether drug effects recorded in both centers were comparable. These analyses revealed that changes in subjective mood following cannabis administration were generally more intense in subjects that participated in the Nijmegen study center as compared to the Maastricht center. We speculate that THC influence was larger in the Nijmegen sample due to differences in cannabis use history and mean THC concentrations that were observed between both centers. Subjects in the Nijmegen sample were (somewhat) less experienced while their mean THC concentration during cannabis treatment was higher as compared to the Maastricht sample. Correlation analyses indicated that positive feelings |(i.e. friendliness, arousal, positive mood) during THC intoxication were positively correlated with Cannabis use history, while negative feelings (i.e. anxiety, depression, anger, fatigue and confusion) were inversely related to history of use. This finding fits with previous research showing that the magnitude of acute THC intoxication decreases with increasing cannabis experience (Ramaekers, Kauert, et al., 2009). Analyses of pharmacokinetic data furthermore revealed that mean THC concentrations in subjects from the Nijmegen center were about twice as high relative to the Maastricht center. Explanations for the observed difference in THC concentrations are all speculative but might include variations in titration of THC vapor, number of inhalations and duration of inhalation. Though THC administration protocols were identical in both centers, adherence may have differed. However, no significant correlations between THC concentration and magnitude of psychedelic experience were found. It has been shown previously (Ramaekers et al., 2006) that correlations between THC concentration and magnitude of THC impairments are relatively low due to large inter-individual variations. Yet, 
in general it is also clear that the number of subjects that respond to THC intoxication increase with dose and concentration (Ramaekers et al., 2006). The present finding that the mean THC concentration in the Nijmegen sample was twice as high as compared to the Maastricht sample may therefore in part explain why the former appeared more sensitive to the effects of THC.

In conclusion, the present study demonstrated that drug users with high trait levels of impulsivity were more sensitive to the psychedelics effects of cannabis and more sensitive to dissociative effects of cocaine. These data support the notion that trait impulsivity contributes to subjective experiences of drug use and the risk of developing drug use problems. 
54 | 


\section{CHAPTER 4}

\section{REWARD RELATED BRAIN ACTIVITY AND TASK PERFORMANCE DURING CANNABIS AND COCAINE INTOXICATION}




\section{Summary}

Drugs of abuse cause their reinforcing effects through dopaminergic stimulation of the limbic reward system but separate drugs may have a different rewarding potential. The present study was designed to assess reward sensitivity in heavy cannabis users during acute challenges with cannabis and cocaine. In total, 50 heavy cannabis users were included and received doses of cocaine $\mathrm{HCl} 300 \mathrm{mg}$, THC $450 \mu \mathrm{g} / \mathrm{kg}$ bodyweight (divided over 2 successive administrations) and placebo in a double blind, 3-way, cross-over study. Reward anticipation was assessed using a Monetary Incentive Delay Task (MIDT) during functional imaging. Reward cues and targets primarily stimulated BOLD activation in the thalamic region and the superior parietal lobule, but not the ventral striatum. Both cannabis and cocaine reduced thalamic activation indicating a loss of sensory gating and an increase in implicit, striatal processing of reward. Behavioral data showed that MIDT performance increased following cocaine as indicated by faster reaction times and higher accuracy, but decreased following cannabis administration as indicated by slower reaction times and lower accuracy. Drug induced performance changes in the MIDT however did not differ between reward and no reward trials. Thalamic decrease in activation was significantly correlated with improved performance following cocaine and with impaired performance following cannabis. It is concluded that performance changes following cannabis and cocaine appear to reflect overall changes in speed and accuracy of psychomotor function rather than a change in reward sensitivity. The decrease in activation of the thalamus following both drugs suggested a reduction in sensory gating, leading to an increase in striatal processing of sensory information and a reduction of higher cognitive control. 


\section{Introduction}

All drugs of abuse cause their reinforcing effects through direct or indirect increase of dopamine neurotransmission in the mesolimbic pathway (Bonci, Bernardi, Grillner, \& Mercuri, 2003; Nestler, 2005; Pierce \& Kumaresan, 2006). The limbic 'reward' circuitry receives modulatory input from ascending dopaminergic projections from the ventral tegmental area (Yacubian \& Büchel, 2009). The reward circuit involves a network of brain structures including the nucleus accumbens, ventral pallidum, medial dorsal nucleus, amygdala, hippocampus, anterior cingulate and the ventral orbital cortex that are involved in reward processing, motivation, salience attribution and cognitive control. Dopaminergic activations of brain "reward" systems however may alter following chronic drug use as a consequence of neuroadaptations in the limbic circuit (Kalivas \& Volkow, 2005; Volkow, Wang, Fowler, Tomasi, \& Telang, 2011).

Examples of such changes in dopaminergic signalling have been shown following prolonged use of cannabis and cocaine. A challenge with $\triangle 9$-tetrahydrocannabinol (THC, the active ingredient of cannabis) increased dopamine concentrations in the limbic circuit of rats with no cannabis use history, but decreased dopamine levels in rats that were frequently exposed to this drug (Jentsch, Verrico, Le, \& Roth, 1998; Pistis, Porcu, Melis, Diana, \& Gessa, 2001; Verrico et al., 2003). Brain imaging studies on striatal dopamine release in humans during a challenge with THC provided mixed results (i.e. increase or decrease of dopamine release or no effect), possibly because they did not control for cannabis use history of the participants (Barkus et al., 2011; Bossong et al., 2009; Kuepper et al., 2013; Stokes et al., 2009). The normal effect of an acute challenge with cocaine is increased dopamine concentrations in the "reward" circuit due to reduced dopamine re-uptake (Bonci et al., 2003; Di Chiara \& Imperato, 1988). Yet, chronic cocaine administration has also been associated with hyperpolarization of dopaminergic neurons due to repeated $D_{2}$ autoreceptor activation leading to a decreased firing rate (Bonci et al., 2003). It has been speculated that altered levels of dopaminergic signalling by the mesencephalic dopaminergic neurons following drug exposure replaces the original physiological state of the reward circuit and that maintenance of the changed state leads to dysregulated sensitivity for reward and continued drug use (Bonci et al., 2003; Volkow et al., 2011).

The Monetary Incentive Delay Task (MIDT) has been used as measure to assess reward anticipation. In this task, increments in Blood Oxygen Level Dependent (BOLD) activations 
have been shown in parts of the reward circuit such as ventral striatum, caudate and thalamus in anticipation of reward (Bjork et al., 2004; Haber \& Knutson, 2010; Knutson, Fong, Adams, Varner, \& Hommer, 2001; Urban, Slifstein, Meda, et al., 2012). Drugs of abuse have also been shown to directly affect the anticipation of monetary rewards on the MIDT. Evidence for striatal dopaminergic dysfunction in drug users as compared to controls was provided by a number of studies investigating the acute effects of drugs on BOLD activation during the MIDT. Single dose administration of dextroamphetamine blunted the peak magnitude of striatal activation in healthy volunteers but extended its duration during anticipation of reward (Knutson et al., 2004). Furthermore, methylphenidate reduced striatal activation on the MIDT when anticipating reward in healthy controls but not in recreational amphetamine users (Schouw et al., 2013). THC also decreased striatal activation in the MIDT in nicotine addicts, but not in (non-smoking) healthy controls which was hypothesized to be due to prodromal differences in endocannabinoid function (Jansma et al., 2013; van Hell et al., 2012). This suggests that the effect of drugs on the BOLD response on the MIDT may be different in drug users compared to controls. Long-term dysregulated sensitivity to monetary reward has been demonstrated in cocaine addicts (Goldstein et al., 2007; Parvaz et al., 2012). Decrements in dopaminergic signaling in drug users might reflect alterations in reward cue sensitivity which in turn may contribute to sustained problematic drug use. This would suggest that the addictive potential of drugs may depend, at least in part, on their intrinsic ability to increase reward sensitivity.

The present study was designed to assess reward sensitivity in heavy cannabis users during acute challenges with cannabis and cocaine. Based on previous findings, it was speculated that cannabis would reduce dopaminergic signalling given the chronic cannabis use history of the participants and that cocaine would increase dopaminergic output in the reward circuit. Consequently, it was expected that these drugs would produce opposing effects on reward anticipation.

\section{Materials \& methods}

\section{Participants}

A total of 50 participants (39 male, 11 female), mean (SD) age 23.23 (3.22) were included. Participants were recruited through advertisements and word-of-mouth. Inclusion criteria were: age 18-40 yrs; regular cannabis use: $\geq 2$ times/week; cocaine use $>5$ times in the 
previous year; free from psychotropic medication; good physical health; normal weight (BMI 18-28) and written informed consent. Exclusion criteria were: dependence on cocaine according to DSM-IV criteria; presence or history of psychiatric or neurological disorder as assessed during a clinical interview; pregnancy or lactation; cardiovascular abnormalities as measured by EKG; hypertension and excessive drinking ( $>20$ units per week) or smoking (>20 cigarettes per day). Additionally, all participants were screened for fMRI compatibility. All had normal or corrected-to-normal vision, were free from neurological abnormalities, did not use medication that could influence cognitive functioning and were screened for MRI contra-indications. A summary of subject demographics and their drug use history is given in Table 1.

\begin{tabular}{lrrrrr}
\hline & Mean & \multicolumn{1}{c}{ SD } & Min & Max & $\begin{array}{c}\text { Duration of } \\
\text { drug use (yrs) }\end{array}$ \\
\hline Age (years) & 23.23 & 3.22 & 18.3 & 32.4 & \\
Weight (kg) & 68.14 & 10.06 & 51 & 91 & \\
Lifetime drug & & & & & \\
use (\# of & & & & & \\
times) & & & & & 7.15 \\
Cannabis & 453.87 & 432.57 & 40 & 2100 & 3.51 \\
Cocaine & 35.41 & 46.62 & 4 & 200 & 8.65 \\
Alcohol & 529 & 378.59 & 50 & 1500 & 4.37 \\
Ecstasy & 25.59 & 27.81 & 1 & 100 & 3.09 \\
Amphetamines & 53.52 & 190.84 & 1 & 1000 & 2.88 \\
Mushrooms & 7.68 & 15.06 & 1 & 75 & 1.67 \\
LSD & 8.78 & 7.87 & 1 & 20 & 1.91 \\
Other & 19.85 & 40.04 & 1 & 150 & \\
\hline
\end{tabular}

Table 1: Mean (SD) age, weight, lifetime drug use (total number of times used) and drug use in years.

This study was part of a larger trial on the association between drug use and impulse control (see trial registration NTR2127 at the Netherlands Trial Register) conducted according to the code of ethics on human experimentation established by the declaration of Helsinki (1964) and amended in Seoul (2008) and was approved by the Medical Ethics Committee of the Academic Hospital of Maastricht and Maastricht University. A permit for obtaining, storing and administering cocaine and cannabis was obtained from the Dutch drug enforcement administration. Subjects were paid for their participation in the study. 


\section{Experimental design}

Subjects participated in a double-blind, placebo-controlled, 3-way crossover study. Treatments consisted of placebo, $450 \mu \mathrm{g} / \mathrm{kg}$ THC and $300 \mathrm{mg}$ cocaine $\mathrm{HCl}$. All testing days started with a urine drug screen and a breath alcohol screen; female subjects underwent an additional pregnancy test. If subjects tested negative (except cannabis) on all screens they could proceed with the testing day. After a standardized breakfast, subjects received a capsule containing either $300 \mathrm{mg}$ cocaine $\mathrm{HCl}$ or placebo orally (T1). Forty-five minutes after capsule administration, subjects inhaled $300 \mu \mathrm{g} / \mathrm{kg}$ bodyweight THC or placebo (T2). Between $\mathrm{T} 1$ and T2, subjects were allowed to read a book or watch television. Between 15-60 minutes following T2 a behavioural test-battery was conducted (see van Wel et al., in press). This was followed by a booster inhalation of $150 \mu \mathrm{g} / \mathrm{kg}$ bodyweight THC or placebo (T3), after which subjects were immediately taken to the $\mathrm{fMRI}$ scanner and performed the Monetary Incentive Delay task. Prior to experimental sessions, subjects were familiarized with procedures and tasks. Subjects had to refrain from all drugs of abuse (except cannabis) at least a week before the start of the experiment until study end.

\section{Monetary Incentive Delay task}

To activate reward circuitry an adapted version of the Monetary Incentive Delay task (MIDT) as developed by Knutson and colleagues (Knutson, Westdorp, Kaiser, \& Hommer, 2000) was used (see (Hermans et al., 2010)). A red or green colored square cue was presented for 3.58.5 seconds. Subsequently, a white circle appeared (the target stimulus), to which subjects invariably had to respond as quickly as possible by pressing a button with their right index finger. A red square cue always predicted no-reward trials $(n=25)$, while green square cues predicted reward $(n=25)$. Additionally, 25 null trials were included where subjects had to respond to cues as normal, but would never get a reward. If subjects responded fast enough to the target following a reward cue, they could win 20 cents. The time-window in which subjects could react increased after misses $(+10 \mathrm{~ms})$ or decreased $(-20 \mathrm{~ms})$ after hits, with a start time-window of 270 ms. This results in a hit-rate of approximately $33 \%$ in both conditions. Subjects received feedback on their performance 2-2.5 seconds after presentation of the target. There was a $500 \mathrm{~ms}$ delay between trials. Total task time varied between 10 and 16 minutes. BOLD activity was assessed for 8 regressors: (1) reward and (2) no-reward cues; (3) reward and (4) no-reward targets; (5) reward-hit, (6) reward-miss, (7) 
no-reward hit and (8) no-reward miss trials. Behavioral variables of interest were reaction time after a cue and the number of hits after a cue.

\section{Analysis of fMRI data}

Event-related $\mathrm{fMRI}$ was performed in a $3 \mathrm{~T}$ head-only scanner (Siemens ${ }^{\circledR}$ MAGNETOM Allegra). A total of 32 slices covering the whole brain were collected using gradient-echo echo-planar imaging (GE-EPI, TR=2000 ms, TE=30 ms, flip angle $=90^{\circ}$, matrix $=64 \times 64$, voxel size=3.5 × $3.5 \times 3.5 \mathrm{~mm}$ ). For anatomical reference, a 3D MPRAGE (magnetization-prepared rapid gradient echo; $\mathrm{TR}=9.7 \mathrm{~ms}, \mathrm{TE}=4 \mathrm{~ms}$, flip angle $=12^{\circ}$, matrix $=256 \times 256$, voxel size $=1 \times 1 \times 1$ $\mathrm{mm} 3$ ) image data set was acquired.

The fMRI data were preprocessed using SPM5 software (Welcome Trust Center for Neuroimaging, London, UK). Data were realigned, slice time corrected, co-registered with the anatomical scan and normalized. Individual T1-weighted anatomical scans from each subject were used for the co-registration with the functional volumes collected from the same subject. T1-weighted anatomical scans were segmented and normalized using SPM5. Functional volumes were subsequently resampled to $3 \mathrm{~mm}$ isotropic voxels and smoothed with a $6 \mathrm{~mm}$ FWHM kernel.

\section{Statistics}

First, overall brain activation during task performance was determined during drug and placebo separately, to obtain a general descriptive view of combined task and drug activations for each condition ( $p<.05$; FDR corrected). A first level analysis was done where all task parameters (reward and no reward cues, reward and no reward targets) were modelled as regressors of interest in a GLM analysis, for each treatment separately. The six realignment parameters were modelled as regressors of no interest.

Subsequent analyses were conducted to specify drug effects in brain areas associated with reward. A first level analysis was conducted to determine task activation during the MIDT in the placebo and drug conditions. Two dependent parameters, i.e. brain activation following a reward cue and following response feedback of positive reward, were modelled as main regressors of interests in a SPM GLM analysis to increase the chance of finding reward-related brain activity. Non-reward cues, non-reward hits, misses, error trials as well as the six realignment parameters were modelled as regressors of no interest. Brain areas 
that were significantly ( $p<.05$, FDR corrected) associated with reward at group level during placebo were defined as prime region of interest (ROI) for studying drug effects on task performance. A second contrast on the individual level subtracted brain activation following a reward cue from brain activation following a no-reward cue. Reward and no-reward hits, misses, error trials as well as the six realignment parameters were modelled as regressors of no interest. Since striatal activation was expected, the striatum was defined as ROI using the WFU PickAtlas (Maldjian, Laurienti, \& Burdette, 2004; Maldjian, Laurienti, Kraft, \& Burdette, 2003).

Mean \% BOLD signal change was determined for each of the identified ROls using the MarsBar toolbox. The overall treatment effect on mean \% BOLD signal change was determined using a GLM repeated measures ANOVA in each ROI. Drug-placebo contrasts were conducted to establish the contribution of cannabis and cocaine to the overall treatment effect. Correlational analysis between \% BOLD signal change and reward related performance measures in each treatment condition were conducted to confirm and support causality between drug-induced changes in BOLD activation and changes in task performance.

All neurocognitive measures were analyzed with SPSS 18.0 using a GLM univariate analysis of variance (ANOVA) with Treatment (3 levels) and Cue (2 levels, reward-no reward) as within subject factors. If the sphericity assumption was violated, the Greenhouse-Geisser correction was used. In case of significant main effect of Treatment, separate drug-placebo contrasts were conducted. The $\alpha$-criterion significance level was set to 0.05 .

\section{Pharmacokinetic assessments}

Blood samples to assess drug concentrations were taken before and after scanning; i.e. 1 minute and 1 hour after smoking the booster cannabis dose (T3) respectively. Blood samples were centrifuged and the resulting plasma and serum was frozen at $-20^{\circ} \mathrm{C}$ until analysis for pharmacokinetic assessments. For cannabinoid determinations serum was used (Serum-Gel Vacuette system of Greiner Bio-One, Alphen a/d Rijn), cocaine and metabolites were determined in plasma (Glucose FX Vacuette system containing $2.5 \mathrm{mg} / \mathrm{ml}$ sodium fluoride and $2 \mathrm{mg} / \mathrm{ml}$ potassium oxalate). The determination of $\Delta^{9}$-Tetrahydrocannabinol (THC), 11hydroxy-THC (THC-OH), 11-nor-9-carboxy-THC (THC-COOH), cocaine (COC), benzoylecgonine 
(BZE) and ecgonine methyl ester (EME) was performed in a specialized forensic-toxicological laboratory using validated procedures (Toennes et al., 2008, 2005).

\section{Results}

Only complete data sets entered data analysis. In total 46 complete datasets were collected. Incomplete data sets resulted from failures to perform the task during drug treatment (2) and drop-out (2). In addition, fMRI recordings of 9 participants had to be excluded due to excessive head movement and signal variability. Therefore, $\mathrm{fMRI}$ analyses were conducted on complete data sets collected from 37 subjects.

\section{Behavioral data MID task}

Mean (SE) performance scores in the MID-task in each treatment condition are shown in

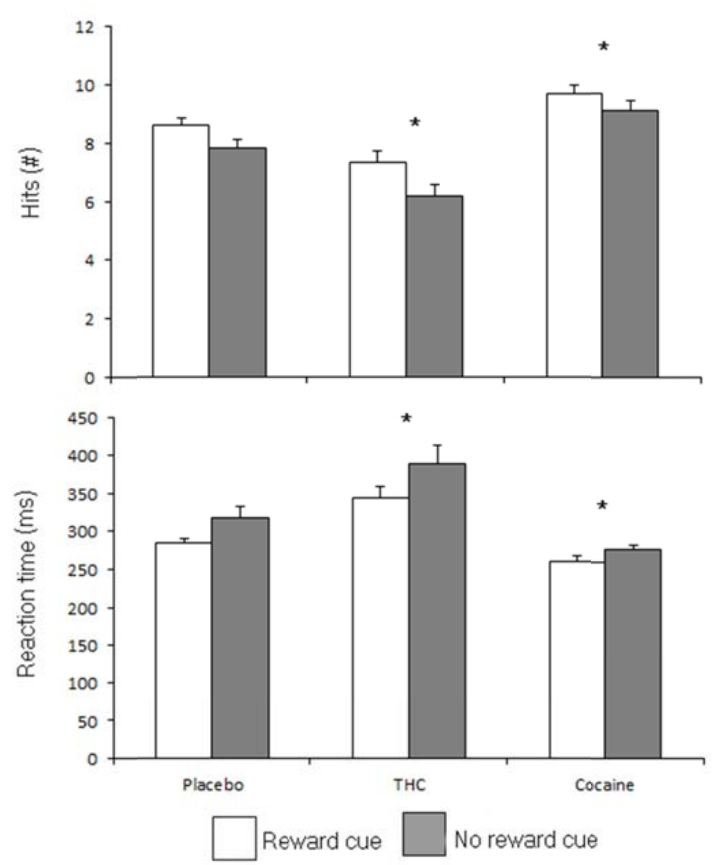

Figure 1: Number (SE) of hits following reward cues and no-reward cues (top) and mean (SE) reaction time after reward and no reward cues (bottom) following placebo, cannabis and cocaine $(*=$ drugplacebo contrast for factor Treatment $p<.05)$.
Figure 1. A significant main effect of Treatment was shown on RT $\left(F_{1,44}=22.99 ; p<.001\right)$ and number of hits $\left(F_{1,44}=40.56 ; \quad p<.001\right)$ Drug-placebo contrasts showed that cocaine significantly decreased RT $\left(F_{1,44}=8.69\right.$; $p=.005$ ) while increasing the number of hits $\left(F_{1,44}=20.38 ; p<.001\right)$. Cannabis had the opposite effect and significantly increased RT $\left(F_{1,44}=15.03 ; p<.001\right)$ while decreasing the number of hits $\left(F_{1}\right.$, 44=22.19; $p<.001$ ). The factor Cue also significantly affected RT $\left(F_{1,44}=17.63\right.$; $p<.001)$ and number of hits $\left(F_{1,44}=13.62\right.$; $\mathrm{p}=.001$ ). Mean RT following reward cues was faster as compared to no reward cues. Mean number of hits were higher following reward cues as compared to no reward cues. Mean differences in RT 
and number of hits following reward and no-reward cues were treatment unspecific as indicated by a non-significant Cue $x$ Treatment interaction.

\section{Imaging data}

Overall BOLD activation during task performance observed during drug and placebo treatment are shown in Figure 2. Major brain regions showing activation during task performance during placebo treatment are summarized in Table 2. Overall BOLD activations did not significantly differ between treatments.

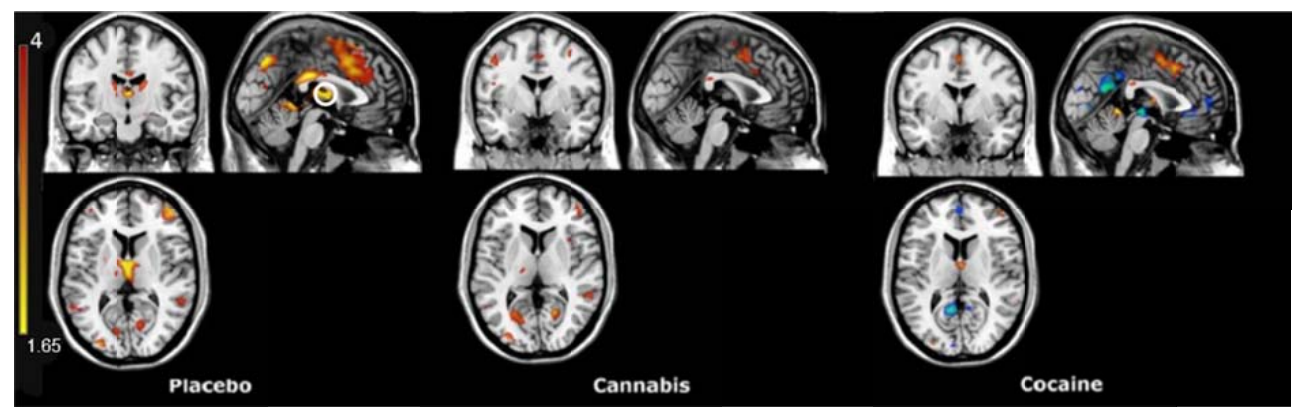

Figure 2: Overall BOLD activation (red) and deactivation (blue) on the MIDT illustrative of placebo, cannabis and cocaine intoxication. Images are shown in neurological convention (left=left, right=right). Overall bold activation did not differ significantly between treatments (FDR corrected). A white circle in the placebo condition indicates the area selected as ROI.

\begin{tabular}{lrrrrrrr}
\hline & & \multicolumn{6}{c}{ MNI } \\
Area & Voxels & $\mathbf{T}_{\max }$ & $\mathbf{p}$ & \multicolumn{3}{c}{ coordinates } \\
& & & & $\mathrm{X}$ & $\mathrm{Y}$ & $\mathrm{Z}$ \\
\hline Thalamus & 14 & 4.87 & 0.038 & 0 & -4 & 8 \\
R Superior Parietal Lobula & 19 & 4.35 & 0.036 & 38 & -66 & 48 \\
R Inferior Parietal Lobula & 13 & 4.34 & 0.038 & 48 & -54 & 54 \\
R Precuneus & 4 & 4.32 & 0.044 & 2 & -74 & 44 \\
R Inferior Parietal Lobula & 2 & 4.20 & 0.046 & 52 & -40 & 56 \\
\hline
\end{tabular}

Table 2: Major brain regions activated during MIDT performance after the placebo treatment. $\mathrm{R}=$ right.

Group contrasts of reward vs no reward cues did not reveal any significant task activation in any brain area. 
Group analysis of BOLD response following reward cues and reward targets (hits) during placebo treatment revealed task activation in two major brain areas (FDR corrected); i.e. the thalamic region ( $\mathrm{MNI}$ coordinates xyz $2-1012$, cluster size $=17$ ) and the superior parietal lobule (MNI coordinates xyz 38 -66 50, cluster size=18). These clusters were identified as relevant reward related ROls for assessing drug effects on reward anticipation and performance. A GLM repeated measures analysis revealed an overall Treatment effect on mean BOLD signal change in the thalamus, but not in the superior parietal lobule. Drugplacebo contrast revealed that cannabis $\left(F_{1,36}=3.93, p=.05\right)$ as well as cocaine $\left(F_{1,36}=5.15, p=\right.$ .03) decreased mean signal change in the thalamus relative to placebo. Mean BOLD signal changes in every treatment condition are shown in Figure 3.

Correlational analysis revealed significant associations between thalamic activation and task performance in both drug conditions. Following cocaine, mean percentage BOLD signal change was positively correlated to mean reaction time $(r=.44, p=.008)$ and negatively correlated to the number of correct hits in reward target trials $(r=-.46, p=.005)$. Following cannabis, mean \% BOLD signal change was negatively correlated to reaction time $(r=-.40$, $\mathrm{p}=.016)$. Reaction time and number of hits were not associated with changes in brain activation during placebo treatment. A scatterplot showing associations between thalamic brain activation and reaction time in all treatment conditions is shown in Figure 3.

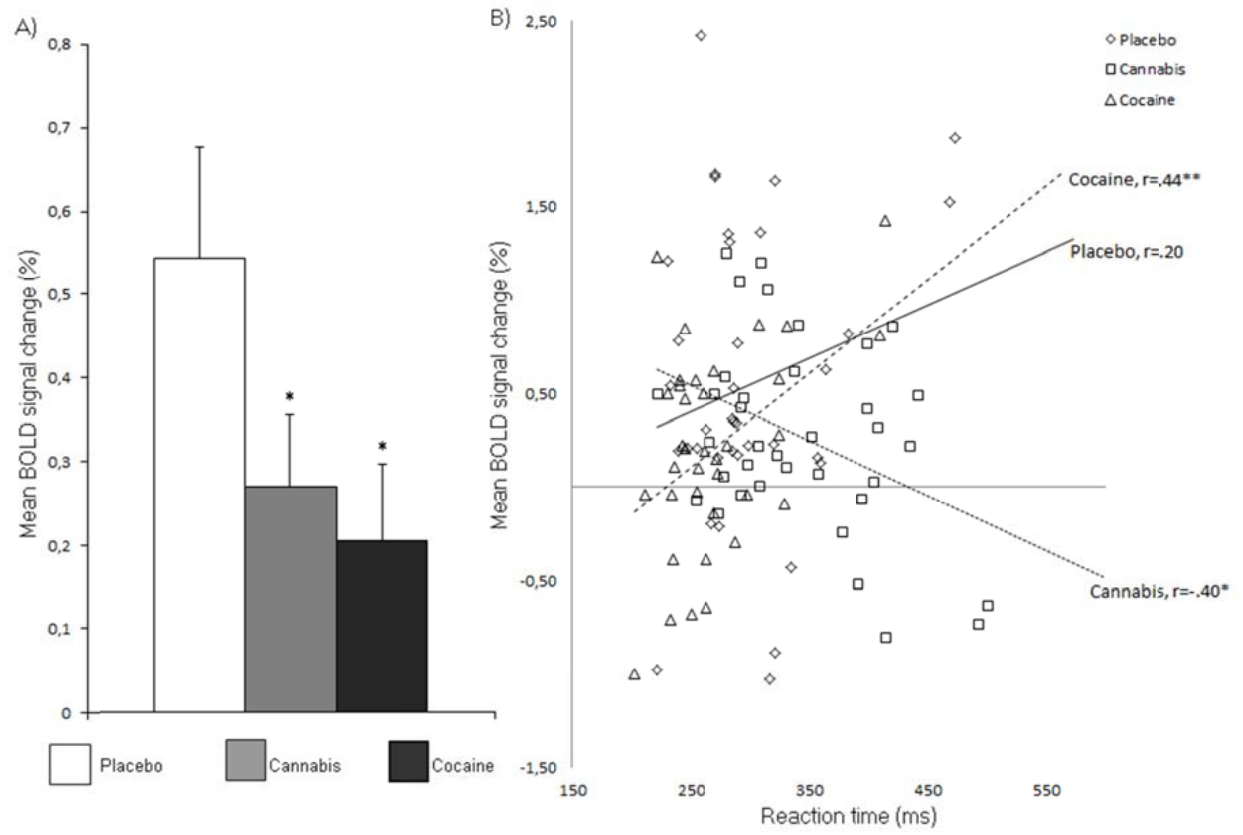

Figure 3: Mean (SE) per cent signal change in the thalamus following placebo, cannabis and cocaine (A). Correlations between \% BOLD signal change and mean RT in the MID task in each treatment condition $(B)(*=p<05 ; * *=p<.001)$. 


\section{Pharmacokinetics}

Mean concentrations of THC, cocaine and their metabolites are shown in table 3 . The cocaine concentrations observed after ingestion are in accordance with previous publications (Wilkinson et al., 1980). Also the cannabinoid concentrations are in the range that has been observed in previous studies with similar THC doses (Toennes et al., 2011).

\begin{tabular}{lllllll}
\hline & THC $[\mu \mathrm{g} / \mathrm{L}]$ & $\begin{array}{l}\text { THC-OH } \\
{[\mu \mathrm{g} / \mathrm{L}]}\end{array}$ & $\begin{array}{l}\text { THC-COOH } \\
{[\mu \mathrm{g} / \mathrm{L}]}\end{array}$ & $\begin{array}{l}\text { Cocaine } \\
{[\mathrm{mg} / \mathrm{L}]}\end{array}$ & $\begin{array}{l}\text { Benzoyl- } \\
\text { ecgonine } \\
{[\mathrm{mg} / \mathrm{L}]}\end{array}$ & $\begin{array}{l}\text { Ecgonine- } \\
\text { methyl- } \\
\text { ester } \\
{[\mathrm{mg} / \mathrm{L}]}\end{array}$ \\
Timepoint & & & & & & $0(.00)$ \\
\hline Baseline & $1.63(3.62)$ & $.73(1.64)$ & $23.28(39.41)$ & $0(.00)$ & $0(.00)$ & $0(.00)$ \\
before MIDT & $37.89(19.94)$ & $5.89(3.35)$ & $41.34(30.97)$ & $.30(.16)$ & $1.02(.37)$ & $.25(.11)$ \\
after MIDT & $8.20(4.76)$ & $3.15(1.72)$ & $34.29(30.77)$ & $.16(.09)$ & $1.33(.54)$ & $.25(.09)$ \\
\hline
\end{tabular}

Table 3: Mean (SD) plasma concentration of THC, cocaine and their metabolites at baseline and before and after the MIDT

\section{Discussion}

The present study was designed to assess reward sensitivity in heavy cannabis users during acute challenges with cannabis and cocaine. It was hypothesized that cannabis and cocaine would affect reward anticipation in the MIDT in opposing ways by modifying underlying dopaminergic mechanisms.

Overall brain activations during MIDT performance following placebo treatment were most prominent in the thalamus and the inferior and superior parietal lobules. Subsequent group analysis of BOLD response following reward cues and reward targets primarily revealed task activation in the thalamic region and the superior parietal lobule. Surprisingly, although previous studies using the MIDT task mainly reported striatal activation, particularly in the nucleus accumbens (Jia et al., 2011; Knutson et al., 2001, 2000; Schouw et al., 2013), we did not find any activation in this area. This is possibly due to the fact that these studies used less stringent corrections for multiple comparisons as compared to the FDR procedure that was applied in the present study. Alternatively, our subjects may have exhibited blunted striatal activation due to their chronic cannabis use history (Urban, Slifstein, Thompson, et al., 2012; Volkow et al., 2012). A similar study by Schouw et al. (2013) showed that methylphenidate decreased striatal activation during a reward anticipation task in healthy controls but not in regular amphetamine users. This may suggest that neuroadaptation in the striatum of chronic drug users decreases the likelihood of measuring 
striatal change during reward manipulations in general. The lack of striatal activation in our study could also indicate that the task manipulation of reward anticipation had failed. However, analyses of behavioral data during MIDT performance revealed that subjects responded faster following reward cues as compared to non-reward cues, independent of treatment condition. This strongly suggests that the experimental manipulation of reward in the present study was effective.

Thalamic activation during MIDT performance fits with its well-described relay function in the reward circuit to gate information flow from the striatum to the cortex (Haber \& Knutson, 2010; Knutson \& Greer, 2008; Liu, Hairston, Schrier, \& Fan, 2011). BOLD activation of the superior and inferior parietal lobules has been associated with risky decision making during reward anticipation, impulse control and self-action monitoring (Congdon \& Canli, 2008; De Bellis et al., 2013; Horn, Dolan, Elliott, Deakin, \& Woodruff, 2003; Ramaekers, Kuypers, Wingen, Heinecke, \& Formisano, 2009). In the current study it was shown that cannabis and cocaine both decreased activation in the thalamic region, but did not affect activation in the superior parietal lobules. These data suggest that both drugs interfered with information processing regulated by thalamic gating.

Thalamic deactivation or activation depends on the functioning of three pathways which loop from the frontal lobes through the striatum to the thalamus and back. These thalamocortical pathways can be classified as hyperdirect, indirect and direct (Frank, 2005). The hyperdirect thalamocortical pathway serves as a braking system prior to selecting a response. The braking system functions by increasing activity in the subthalamic nucleus (STN), which has a direct excitatory action on the internal globus pallidus (GPi). This inhibits the thalamus which prevents a motor response from occurring (Nambu, Tokuno, \& Takada, 2002). The hyperdirect pathway thus is driven by the frontal (motor) cortex and bypasses the striatum. Its major role is to activate sensory gating of the thalamus in order to suppress erroneous responses. Dysfunction in the hyperdirect pathway has been associated with a loss of cognitive control leading to erroneous responses, increased impulsivity and even addiction (Baunez \& Lardeux, 2011; Pierce \& Vassoler, 2013). The indirect pathway originates from striatopallidal cells and passes through the external globus pallidus (GPe). This disinhibits the GPi, by which the thalamus gets more inhibition and therefore less signals are transmitted to the frontal cortex. The direct pathway receives activation from striatonigral cells, which have an inhibitory action on the GPi. This then disinhibits thalamic 
control over behavior, causing excitatory signals to be sent to the frontal cortex (Cohen \& Frank, 2009). The thalamic activation observed in the placebo condition reflects the net outcome of inhibitory and excitatory projection from the three pathways. BOLD activation in the thalamus therefore would represent an effective and selective gating mechanism that updates and directs operations that are then fed back to the frontal lobes through the thalamus. Deactivation of the thalamus has been associated with reduced sensory gating, leading to an increase in striatal processing of sensory information and a reduction of higher cognitive control (Cho et al., 2013; Schiff, 2008; Van der Werf, Witter, \& Groenewegen, 2002). Likewise, the reduction in thalamic BOLD activation during cannabis and cocaine intoxication would also be expected to indicate a shift toward striatal processing of sensory information resulting from a drug induced loss of 'hyperdirect' cognitive control.

The decrease in thalamic activation during cannabis and cocaine was significantly correlated with performance changes during the MIDT. The decrease in thalamic activation following cocaine was significantly correlated with shorter reaction times and higher accuracy following cocaine and with longer reaction times following cannabis. The drug effects on behaviour appear in line with previous research showing that acute doses of cocaine can improve psychomotor and cognitive performance whereas cannabis produces performance impairment (Burns, 1991, 1992; Fillmore et al., 2002, 2006; Garavan et al., 2008; Metrik et al., 2012; Ramaekers, Kauert, et al., 2009; Spronk, van Wel, Ramaekers, \& Verkes, 2013). Thus, behavioral data indicates that psychomotor functioning is increased after cocaine administration, as indicated by faster reaction times and more accuracy, but decreased after cannabis administration as indicated by slower reaction times and lower accuracy. More importantly, the behavioral effects of cocaine and cannabis were similar following reward and no reward cues indicating that none of the drugs actually altered reward sensitivity or anticipation selectively.

The finding that a decrease in thalamic activation is correlated to improvement of performance during cocaine intoxication as well as impairment of performance during cannabis intoxication appears contradictory but might directly follow from the neuropharmacological profiles of these drugs. Cocaine increases dopaminergic signaling in the striatum. This might cause the drug to increase activity in the direct pathway after the initial inhibition of behavior by the hyperdirect pathway. This would explain why cocaine administration increases the speed and accuracy of responding. Cannabis on the other hand 
decreases dopaminergic tone. A decrease in dopamine has been shown to stimulate the inhibitory, indirect pathway, thereby decreasing the likelihood of a response occurring. Apart from the opposing effect on direct and indirect pathways, cocaine and cannabis would also be expected to produce identical effects on the hyperdirect pathway, which has been associated with thalamic gating. It has been shown that drugs of abuse decrease thalamic gating as evidenced by studies showing alterations in pre-pulse inhibition after drug administration (Ding, Guzman, Peterson, Goldberg, \& Surmeier, 2010; Dissanayake, Mason, \& Marsden, 2013; Green, Mechan, Elliott, Shea, \& Colado, 2003). The decrease in thalamic gating and its resulting impairments in impulse control might explain the increased impulsivity seen in drug users (de Wit, 2008; Schiff, 2008). In the present study, the reduction in thalamic BOLD activation thus seems to suggest that both cannabis and cocaine had an increased inhibitory effect on early thalamic activity which could not be overcome by the subsequent activation of the direct pathway. The difference in speed between the hyperdirect and indirect/direct pathway has been speculated to be as brief as $22 \mathrm{~ms}$ (Chambers, Garavan, \& Bellgrove, 2009) which currently cannot be picked up by fMRI techniques. This might explain why both drugs seem to have the same effect on thalamic activation while having opposing effects on the behavioral level.

In summary, cannabis and cocaine did not selectively change reward sensitivity during intoxication as measured by the MIDT. Performance changes following cannabis and cocaine were identical following reward and no reward cues and appeared to reflect overall changes in speed and accuracy of psychomotor function. The decrease in activation of the thalamus following both drugs indicated a reduction in sensory gating, leading to an increase in striatal processing of sensory information and a reduction of higher cognitive control. 
70 | 


\title{
CHAPTER 5
}

\section{BLOCKADE OF 5-HT 2 RECEPTOR SELECTIVELY PREVENTS MDMA}

\author{
INDUCED VERBAL MEMORY IMPAIRMENT
}




\section{Summary}

MDMA or "ecstasy" has been associated with memory deficits during abstinence and intoxication. The human neuropharmacology of MDMA induced memory impairment is unknown. This study investigated the role of $5-\mathrm{HT}_{2 \mathrm{~A}}$ and $5-\mathrm{HT}_{1 \mathrm{~A}}$ receptors in MDMA induced memory impairment. Ketanserin is a $5-\mathrm{HT}_{2 \mathrm{~A}}$ receptor blocker and pindolol a $5-\mathrm{HT}_{1 \mathrm{~A}}$ receptor blocker. It was hypothesized that pretreatment with ketanserin and pindolol would protect against MDMA induced memory impairment.

Subjects ( $N=17)$ participated in a double-blind, placebo controlled, within-subject design involving 6 experimental conditions consisting of pretreatment (T1) and treatment (T2). T1 preceded T2 by 30 minutes. T1-T2 combinations were: placebo-placebo, pindolol 20mg-placebo, ketanserin 50mg-placebo, placebo-MDMA 75mg, pindolol 20mg-MDMA $75 \mathrm{mg}$ and ketanserin 50mg-MDMA $75 \mathrm{mg}$. Memory function was assessed at Tmax of MDMA by means of a word learning task, a spatial memory task and a prospective memory task.

MDMA significantly impaired performance in all memory tasks. Pretreatment with a $5-\mathrm{HT}_{2 \mathrm{~A}}$ receptor blocker selectively interacted with subsequent MDMA treatment and prevented MDMA induced impairment in the word learning task, but not in the spatial and prospective memory task. Pretreatment with a $5-\mathrm{HT}_{1 \mathrm{~A}}$ blocker did not affect MDMA induced memory impairment in any of the tasks.

Together, the results demonstrate that MDMA induced impairment of verbal memory as measured in the word learning task is mediated by $5-\mathrm{HT}_{2 \mathrm{~A}}$ receptor stimulation. 


\section{Introduction}

3,4-Methylenedioxymethamphetamine (MDMA) is the primary psychoactive constituent in the popular party drug Ecstasy. Use of MDMA has consistently been associated with learning and verbal memory deficits in recreational and abstinent users (reviews: Cole \& Sumnall, 2003; Morgan, 2000; Parrott, 2001); the prototypical example being a reduction in performance on immediate and delayed word recall tasks. Although the existence of these persistent memory deficits in MDMA users has been well established, their neuropharmacology has been a matter of continuous debate. There is some evidence that these deficits are caused by MDMA induced neurotoxicity as indicated by depleted serotonin (5-HT) levels in MDMA users and by dose response relationships between the extent of exposure to MDMA and the magnitude of memory impairment. Yet, imaging studies demonstrated that memory impairment in ecstasy users was not associated with binding to cortical 5-HT transporters or duration of abstinence, which suggests that memory deficits in MDMA users occur independent of serotonergic neurotoxicity (Reneman et al., 2001).

A similar conclusion was drawn by Kuypers and Ramaekers in their 2005 and 2007 papers. They set out to inquire whether memory performance is directly related to 5-HT synaptic availability. They treated 18 recreational MDMA users with single doses of MDMA $75 \mathrm{mg}$ in a double blind, placebo controlled study and conducted both a verbal memory test and a spatial memory test during intoxication (i.e. 1-2 hrs post drug) and during withdrawal (i.e. 25-26 hrs post drug). Subjects displayed transient memory impairment during intoxication while they performed at placebo level in the withdrawal phase, when serotonin levels were depleted. These results suggest that memory performance during MDMA intoxication is not directly affected by changes in the availability of 5-HT in the synaptic cleft.

The apparent dissociation between general 5-HT levels and MDMA induced memory impairment raises the question whether MDMA exerts its detrimental effect on memory through other mechanisms than synaptic 5-HT-availability alone. Two interesting possibilities include direct and indirect activation of postsynaptic 5- $\mathrm{HT}_{2 \mathrm{~A}}$ and $5-\mathrm{HT}_{1 \mathrm{~A}}$ receptors respectively. It is clear from animal studies that $5-\mathrm{HT}_{2 \mathrm{~A}}$ receptors mediate learning and memory processes (Galizio, McKinney, Cerutti, \& Pitts, 2009; Kay, Harper, \& Hunt, 2010; Trigo, Cabrero-Castel, Berrendero, Maldonado, \& Robledo, 2008) although it is not completely obvious whether $5-\mathrm{HT}_{2 \mathrm{~A}}$ receptor drugs achieve their facilitating and impairing effects through agonism, antagonism or inverse agonism (Meneses, 2002). Still, there 
appears to be some consensus that a $5-\mathrm{HT}_{2 \mathrm{~A}}$ blockade not only improves learning, but reverses poor memory consolidation conditions associated with, among others, dysfunctional serotonergic neurotransmission (Meneses, 2007a, 2007b) as well. 5- $\mathrm{HT}_{2 \mathrm{~A}}$ receptor agonists such as $\mathrm{mCPP}$ have been shown to decrease learning and memory in rats (Meneses, 1999). The same mechanism might be responsible for the decrement in learning and delayed recall following MDMA administration, as it has been previously demonstrated that MDMA possesses a moderate affinity for activating the $5-\mathrm{HT}_{2 \mathrm{~A}}$ receptor (Sadzot et al., 1989).

A second candidate mechanism by which MDMA could cause its disadvantageous effects on memory is indirect activation of the post-synaptic $5-\mathrm{HT}_{1 \mathrm{~A}}$ receptor. The latter has also been shown to modulate memory performance in animals and humans (Liechti et al., 2000; Meneses, 1999; Yasuno et al., 2003). PET studies have shown a negative correlation between memory function and $5-\mathrm{HT}_{1 \mathrm{~A}}$ receptor agonists binding in the hippocampus. More specifically, the 5- $\mathrm{HT}_{1 \mathrm{~A}}$ agonist tandospirone dose dependently impaired performance in an immediate and delayed word recall task (Yasuno et al., 2003). The memory impairing effects of $5-\mathrm{HT}_{1 \mathrm{~A}}$ agonists thus appear identical to those produced by MDMA. It is not known whether MDMA also acts as a direct $5-\mathrm{HT}_{1 \mathrm{~A}}$ receptor agonist. However, it may achieve the same net result by indirectly stimulating postsynaptic $5-\mathrm{HT}_{1 \mathrm{~A}}$ receptors during the short-term increase in availability of synaptic 5-HT for the period of intoxication.

This study was designed to investigate the role of $5-\mathrm{HT}_{2 \mathrm{~A}}$ and $5-\mathrm{HT}_{1 \mathrm{~A}}$ receptors in MDMA induced memory impairment. Recreational MDMA users performed a number of learning and memory tasks after receiving single doses of MDMA in a placebo-controlled study. In line with previous findings and assumptions, it is hypothesized that (1) an acute dose of MDMA will produce impairments on laboratory measures of learning and memory; (2) MDMA-induced memory impairment will be reversed by pre-treatment with ketanserin, a 5- $\mathrm{HT}_{2 \mathrm{~A}}$ receptor antagonist, if impairment is related to direct or indirect stimulation of 5$\mathrm{HT}_{2 \mathrm{~A}}$ receptors; and (3) MDMA- induced memory impairment will be reversed by pretreatment with pindolol, a 5- $\mathrm{HT}_{1 \mathrm{~A}}$ partial agonist, if impairment is related to direct or indirect stimulation of $5-\mathrm{HT}_{1 \mathrm{~A}}$ receptors. 


\section{Materials and methods}

\section{Participants}

A total of 17 healthy MDMA-users (9 male, 8 female), aged between 19 and 27 (mean (SD) $22.76(2.75))$ participated in the study. They were, with the exception of two, all mild to moderate users of MDMA who reported to have taken the drug on 1 - 65 separate occasions (mean 10.94) in the previous year. Overall, subjects reported to have taken MDMA 3 to 780 times in their lifetime (mean 72.4 times). When the heavy users were excluded from the analysis, the mean lifetime use of MDMA dropped to 13 times. Subject demographics are shown in table 1.

\begin{tabular}{lllll}
\hline & Mean & SD & Min & Max \\
\hline Age (years) & 22.76 & 2.75 & 18.6 & 27.2 \\
Height (cm) & 179.6 & 12.3 & 160.0 & 200.0 \\
Weight $(\mathrm{kg})$ & 72.9 & 14.7 & 56.0 & 110.0 \\
Alcohol & 1168.5 & 794.1 & 260.0 & 2600.0 \\
Cannabis & 554.4 & 1104.8 & 0.0 & 3650.0 \\
Ecstasy & 72.4 & 194.7 & 3.0 & 780.0 \\
Amphetamines & 4.1 & 12.3 & 0.0 & 49.0 \\
Cocaine & 4.7 & 7.3 & 0.0 & 25.0 \\
LSD & 4.4 & 12.0 & 0.0 & 50.0 \\
Mushrooms & 0.2 & 0.5 & 0.0 & 2.0 \\
Other & 0.5 & 1.7 & 0.0 & 7.0 \\
\hline
\end{tabular}

Table 1: Subject demographics - drug history (lifetime use in number of times used).

Participants were recruited through advertisements in local newspapers and by word of mouth. Subjects were examined by the medical supervisor, who checked vital signs, inclusion criteria (written informed consent, age 18-35 years, history of MDMA use, free from psychotropic medication; good physical health as assessed by a medical doctor; normal weight as determined by BMI 18-28) and exclusion criteria (addiction according to DSMIV criteria, presence or history of psychiatric or neurological disorder as assessed during a clinical interview; pregnancy; cardiovascular abnormalities; excessive drinking or smoking, that was defined as more than 20 standard units of alcohol per week and more than 10 cigarettes per day, hypertension) and took blood samples for standard blood chemistry and haematology.

This study was conducted according to the code of ethics on human experimentation established by the declaration of Helsinki (1964) and amended in Seoul (2008) and approved 
by the Medical Ethics Committee of the Academic Hospital of Maastricht and Maastricht University. A permit for obtaining, storing and administering MDMA was obtained from the Dutch drug enforcement administration. Subjects were paid for their participation in the study.

\section{Treatments and procedures}

Subjects participated in a double-blind, placebo controlled, within-subject design involving 6 experimental conditions consisting of pretreatment (T1) and treatment (T2). T1 preceded T2 by 30 minutes. T1-T2 combinations were: placebo-placebo, pindolol 20mg-placebo, ketanserin 50mg-placebo, placebo-MDMA 75mg, pindolol 20mg-MDMA 75mg and ketanserin 50mg-MDMA $75 \mathrm{mg}$. Conditions were separated by minimum wash-out period of 7 days to avoid cross-condition contamination. The $75 \mathrm{mg}$ dose of MDMA was selected because it falls within the normal range of recreational use (Gouzoulis-Mayfrank \& Daumann, 2009) and has been consistently shown to impair memory performance and produce robust subjective mood changes in a number of previous studies from our lab (Kuypers, Samyn, \& Ramaekers, 2006; Kuypers \& Ramaekers, 2005, 2007; Ramaekers, Kuypers, et al., 2009). Doses of pindolol 20mg and ketanserin $50 \mathrm{mg}$ represent regular therapeutic doses that block approximately $40 \%$ of $5 \mathrm{HT}_{1 \mathrm{~A}}$ receptors and $91 \%$ of $5 \mathrm{HT}_{2}$ receptors respectively (Brogden \& Sorkin, 1990; Rabiner et al., 2000; Sharpley, Elliott, Attenburrow, \& Cowen, 1994).

Order of conditions was balanced over subjects and sessions. All subjects received a training session before onset of the experimental sessions in order to familiarize them with the tests and procedures. Treatments at T1 and T2 were administered using a double dummy technique to synchronize time of maximal drug concentrations (Tmax). Memory function was assessed by means of a number of memory tasks between 1.5-2 $\mathrm{h}$ after T2 (at Tmax), during which time subjects were allowed to watch TV or read a book. In addition, blood pressure and body temperature were assessed as safety measures. A summary of the testing day is given in Table 2. During the testing day, mood questionnaires and tasks measuring impulsivity were also administered of which the results are discussed elsewhere in this thesis.

Subjects were asked to refrain from drugs at least a week before the start of the experiment and during the study. Subjects were not allowed to use alcohol on the day 
before an experimental session and were requested to arrive at experimental sessions well rested. Drug and alcohol screens were carried out before experimental sessions upon arrival of the subject. Drug screens assessed for the presence of benzodiazepines, opiates, cocaine, marijuana, MDMA and (meth)amphetamine. Women were given a pregnancy test. Study treatments were only administered if subjects tested negative for drugs and pregnancy.

\begin{tabular}{lllll}
\hline 9:00 & 9:30 T1 & 10:00 T2 & 11:30 & 11:30-13:00 \\
\hline Arrival & PLA & PLA & Vital signs & Memory tests \\
& Ketanserin & PLA & measurements & \\
& Pindolol & PLA & and blood & \\
& PLA & MDMA & sample & \\
& Ketanserin & MDMA & collection & \\
& Pindolol & MDMA & & \\
\hline
\end{tabular}

Table 2: Schematic representation of a testing day with 6 treatment conditions. During pretreatment (T1) subjects received either placebo, ketanserin 50mg or pindolol $20 \mathrm{mg}$. Treatment (T2) consisted of either MDMA $75 \mathrm{mg}$ or placebo.

\section{Memory tests}

Three tests with demonstrated sensitivity (Kuypers \& Ramaekers, 2005; Ramaekers, Kuypers, et al., 2009) to the impairing effects of MDMA on memory were included in the memory task battery.

\section{Word learning task}

The Word Learning Task (WLT) is the Dutch language version of the standardized, clinically validated test for verbal memory (Rey, 1958). The test begins with the sequential presentation of 30 monosyllabic common nouns. Each word is shown on the computer display for $2 \mathrm{~s}$ and the subject has to read them out loud. When the series ends, the subject is required to recall as many words as possible. The number correct is scored as the 1st trial score. Thereafter the same list is presented in the same manner on two successive occasions. Numbers correct are scored as before. The main parameter is the sum score of correctly recalled words over three trials. After a 30-min delay, the subject is shown a series of 30 words on the computer display, comprising of 15 words of the original set and 15 new words in random order. He/she was asked to respond as fast as possible to indicate whether the given word was one of the original set. The number of correct recognitions and the 
average speed (ms) of correct recognitions are recorded as the main dependent parameters (Deelman, Liebrand, Koning-Haanstra, \& van den Burg, 1980; Schmitt, Kruizinga, \& Riedel, 2001).

\section{Spatial Memory Task}

The spatial memory task is based on the spatial localization task (Vermeeren et al., 1995). It assesses short-term memory for non-verbal information. The subject is briefly shown a fixation point in the center of the computer display. Shortly thereafter, a target appears at a random location for $500 \mathrm{~ms}$. The subject has to memorize the position of the target and, using a computer mouse, relocate the cursor as accurately as possible over that position. The cursor appears either immediately upon target offset or after a delay of 2 or $4 \mathrm{sec}$. The subject presses a button to indicate that the cursor is at the recalled position of the target. The test consists of 75 trials, divided equally among three response delays. The sequence of delays is random. Mean localization error $(\mathrm{mm})$ and average reaction time $(\mathrm{ms})$ are the main parameters.

\section{Prospective memory task}

The prospective memory task is a new paradigm (Ramaekers, Kuypers, et al., 2009) that was developed to examine prospective memory performance in an event-based memory task. The foreground task consists of 240 successive presentations of a letter ( $A$ or $B$ ) in the center of a computer screen. Subjects are required to respond to each letter as quickly as possible by pressing one of two response buttons. One button is pressed to indicate that the letter " $A$ " appeared and the other to indicate the letter " $B$ ". Both letters are presented equally often. Subjects are informed about the trial number by means of a trial counter that is always present in the left top corner of the screen. In addition, subjects are presented at irregular times with a future trial number in the right top corner of the display. Subjects are instructed to remember this future trial number and withhold from responding to the foreground task during the actual occurrence of the future trial. The memory set of trial numbers is dynamic and contains up to three future trial numbers. A trial number in the memory set is replaced by a novel future trial number whenever the actual trial number matches a future trail number in the set. Trials during which subjects are expected to respond are classified as Go trials. Trials during which subjects are instructed to withhold a 
response are classified as No Go trials (prospective memory trials). Time between presentation of a future trial number and the actual occurrence of the trial (i.e. memory delay) varies between 1, 2 and 3 min, equally divided over all No go trials. In total, the prospective memory task consists of 216 Go trials and 24 No go trials. Number of correct prospective memory recalls (i.e. number of correct response inhibitions) in No go trials is the primary dependent performance parameter.

\section{Pharmacokinetics}

Blood samples were collected before the start of the memory tasks at $1.5 \mathrm{hrs}$ post T2. Blood samples were centrifuged immediately and the serum was subsequently frozen at $-20^{\circ} \mathrm{C}$ until analyses for pharmacokinetic assessments. MDMA, pindolol and ketanserin concentrations were determined using solid phase extraction and gas chromatography with mass spectrometric detection.

\section{Statistics}

The hypotheses that pretreatment with ketanserin or pindolol would interact with MDMA induced memory impairment was tested in 2 separate General Linear Model (GLM) analyses. Memory effects of MDMA, Ketanserin and MDMA x Ketanserin were analyzed by means of a GLM repeated measures ANOVA with MDMA (two levels, i.e. present-absent) and Ketanserin (two levels; present-absent) as the main factors (GLM 1 see Table 2). Memory effects of MDMA, Pindolol and MDMA x Pindolol were analyzed by means of a GLM repeated measures ANOVA with MDMA (two levels, i.e. present-absent) and Pindolol (two levels: present-absent) as the main factors (GLM 2, see Table 2). In case of a significant interaction between MDMA and Ketanserin or MDMA and Pindolol, additional drug-placebo contrasts were conducted in order to further elucidate the nature of the interaction. The alpha criterion significance level was set at $p=0.05$. Safety measures were analyzed in the same manner as measures of memory performance. All statistical tests were conducted with SPSS version 15.0 . 


\section{Results}

In all, 17 complete data sets entered statistical analysis. Statistical analysis of memory data is described in detail below. A summary of main effects on measures of memory performance is given in Table 3.

Word learning task

MDMA significantly decreased immediate recall in both the ketanserin and pindolol comparisons $\left(F_{1,16}=12.1 ; p<.003\right.$ and $F_{1,16}=69.1 ; p<.001$ respectively). Ketanserin did not affect memory but significantly interacted with MDMA to prevent impairment of immediate recall $\left(F_{1,16}=11.7 ; p=.004\right)$. Pindolol neither affected immediate recall nor interacted with the effect of MDMA on memory. Separate drug-placebo contrasts supported ANOVA and revealed memory impairment only after treatment with $\operatorname{MDMA}(p=.001)$ and the combination of pindolol with MDMA $(p=.024)$. Mean $(S E)$ immediate recall scores in each treatment condition are given in Figure 1. Recognition scores were not affected by MDMA, nor by pindolol or ketanserin.

GLM 1: Factors MDMA ( present - absent) and Ketanserin (present - absent)

Treatments included PLA-PLA, PLA-MDMA, KET-PLA and KET-MDMA

\begin{tabular}{|c|c|c|c|c|}
\hline & $\begin{array}{l}\text { WLT } \\
\text { Immediate } \\
\text { recall }\end{array}$ & $\begin{array}{l}\text { PMT } \\
\text { Prospective } \\
\text { recall }\end{array}$ & $\begin{array}{l}\text { SMT } \\
\text { Localisation } \\
\text { error }\end{array}$ & $\begin{array}{l}\text { SMT } \\
\text { Reaction } \\
\text { time }\end{array}$ \\
\hline \multicolumn{5}{|l|}{ Main effects } \\
\hline MDMA & .003 & .013 & .001 & .015 \\
\hline Ketanserin & - & - & .005 & - \\
\hline MDMA x Ketanserin & .004 & - & - & - \\
\hline \multicolumn{4}{|c|}{ GLM 2: Factors MDMA (present - absent) and Pindolol (present - absent) } & Treatments included PLA-PLA, PLA-MDMA, PIN-PLA and PIN-MDMA \\
\hline & $\begin{array}{l}\text { WLT } \\
\text { Immediate } \\
\text { recall }\end{array}$ & $\begin{array}{l}\text { PMT } \\
\text { Prospective } \\
\text { recall }\end{array}$ & $\begin{array}{l}\text { SMT } \\
\text { Localisation } \\
\text { error }\end{array}$ & $\begin{array}{l}\text { SMT } \\
\text { Reaction time }\end{array}$ \\
\hline \multicolumn{5}{|l|}{ Main effects } \\
\hline MDMA & .001 & - & .005 & .001 \\
\hline Pindolol & - & - & - & - \\
\hline MDMA x Pindolol & - & - & - & - \\
\hline
\end{tabular}

Table 3: Summary of main effects and interactions following 2 major GLM analyses for all dependent variables in the Word Learning Task (WLT), the Prospective Memory Task (PMT) and the Spatial Memory Task (SMT). Significance $(p<.05)$ and non-significance $(-)$ of main effects is shown. 


\section{Prospective Memory Task}

MDMA significantly increased the number of prospective memory failures in the NoGo trials in one of the GLM comparisons $\left(F_{1,16}=7.8 ; p=.013\right)$ and showed a trend in the other $\left(F_{1,16}=4\right.$; $\mathrm{p}=.06)$. The factors Ketanserin and Pindolol or their interaction with MDMA did not affect prospective memory. Mean (se) prospective memory recalls as a percentage of the total prospective memory trials in each treatment condition are given in Figure 1.
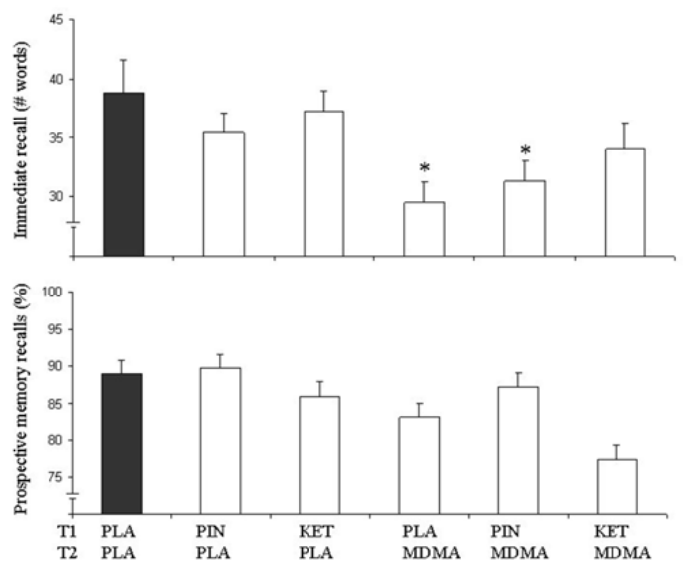

Figure 1: Mean (SE) immediate recall and prospective memory recall in WLT and the PMT for every treatment condition. Each treatment condition consisted of a pretreatment (T1) and a treatment (T2). (PLA=placebo, $\mathrm{PIN}=$ pindolol, $\mathrm{KET}=$ ketanserin, $\mathrm{MDMA}=3,4-$ methylenedioxymethamphetamine, $*=p<.05$ as indicated by drug-placebo contrasts following a significant main interaction effect of MDMA $x$ Ketanserin in the Word Learning Task).

\section{Spatial Memory Task}

MDMA significantly increased localization error in both GLM comparisons $\left(F_{1,16}=19.26\right.$; $p<.001$ and $\left.F_{1,16}=10.4 ; p=.005\right)$. The factor ketanserin also significantly increased localization error $\left(F_{1,16}=10.5 ; p=.005\right)$. MDMA did not interact with any of the pretreatments. In addition, MDMA significantly decreased reaction time in both $G L M$ comparisons $\left(F_{1,16}=7.48\right.$; $\mathrm{p}=.015$ and $\left.\mathrm{F}_{1,16}=32.42 ; \mathrm{p}<.001\right)$. The factors pindolol, ketanserin or their interaction with MDMA did not affect reaction time. Mean localization error and reaction time in all treatment conditions are shown in Figure 2. 

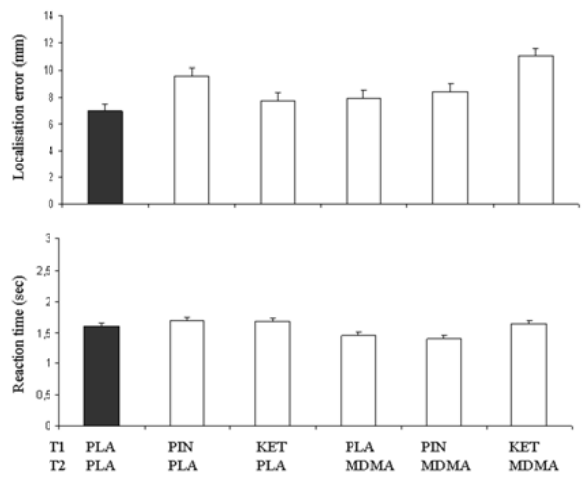

Figure 2: Mean (SE) localisation error and reaction time in the SMT for every treatment condition. Each treatment condition consisted of a pretreatment ( $\mathrm{T} 1)$ and a treatment ( $\mathrm{T} 2)$. (PLA=placebo, $\mathrm{PIN}=$ pindolol, $\mathrm{KET}=$ ketanserin, MDMA=3,4-methylenedioxymethamphetamine).

\section{Safety data}

Safety data revealed a significant main effect of $\operatorname{MDMA}\left(F_{1,16}=24.5 ; p<.001\right)$, pindolol $\left(F_{1,16}=\right.$ 26.7; $\mathrm{p}<.001$ and ketanserin $\left(\mathrm{F}_{1,16}=4.48 ; \mathrm{p}<.05\right)$ on blood pressure. Overall, mean blood pressure ranged from 73.6 to $87.3 \mathrm{mmHg}$ over all treatments. MDMA increased mean blood pressure by $7 \mathrm{mmHg}$ relative to placebo, whereas ketanserin and pindolol mildly reduced mean blood pressure relative to placebo. However, ketanserin and pindolol did not interact with the effect of MDMA on blood pressure. Mean body temperature ranged from $36.2-$ $36.8^{\circ} \mathrm{C}$ over all treatment conditions. Overall, MDMA increased body temperature $\left(\mathrm{F}_{1,16}=\right.$ 4.4; $\mathrm{p}<.05)$. Relative to placebo, body temperature increased by $0.2{ }^{\circ} \mathrm{C}$ after MDMA alone. Pretreatments did not affect body temperature or the effect of MDMA on body temperature.

\section{Pharmacokinetics}

Mean drug concentrations in all treatment conditions are shown in Table 4.

\begin{tabular}{|c|c|c|c|c|c|c|}
\hline $\begin{array}{l}\text { T1 } \\
\text { T2 }\end{array}$ & $\begin{array}{l}\text { PLA } \\
\text { PLA }\end{array}$ & $\begin{array}{l}\text { PIN } \\
\text { PLA }\end{array}$ & $\begin{array}{l}\text { KET } \\
\text { PLA }\end{array}$ & $\begin{array}{l}\text { PLA } \\
\text { MDMA }\end{array}$ & $\begin{array}{l}\text { PIN } \\
\text { MDMA }\end{array}$ & $\begin{array}{l}\text { KET } \\
\text { MDMA }\end{array}$ \\
\hline \multirow[t]{2}{*}{ Dose (mg) } & - & 20 & 50 & - & 20 & 50 \\
\hline & - & - & - & 75 & 75 & 75 \\
\hline \multirow[t]{2}{*}{$\mathrm{C}_{\text {mean }}(\mathrm{ng} / \mathrm{mL})$} & - & $133(80)$ & $86(42)$ & - & $130(53)$ & $104(41)$ \\
\hline & - & - & - & $157(48)$ & $156(56)$ & $164(62)$ \\
\hline
\end{tabular}

Table 4: Dose and mean (SD) drug concentrations in each of the 6 treatment conditions. 


\section{Discussion}

The goal of this study was to investigate the role of $5-\mathrm{HT}_{2 \mathrm{~A}}$ and $5-\mathrm{HT}_{1 \mathrm{~A}}$ receptors in MDMA induced memory impairment. Subjects were given single doses of MDMA combined with placebo, ketanserin or pindolol, after which several learning and memory tasks were carried out.

Single doses of MDMA produced memory impairments in all memory tasks. GLM analyses revealed that overall, MDMA decreased immediate recall in the WLT, increased the number of prospective memory failures in the Prospective Memory Task and increased localisation error in the Spatial Memory Task. These results are fully in line with previous studies that have reported identical memory impairments during intoxication with MDMA (Kuypers \& Ramaekers, 2005, 2007; Ramaekers, Kuypers, et al., 2009) as assessed in identical memory paradigms. These results basically indicate that the current memory paradigms were sensitive to the impairing effects of MDMA and should serve as reliable models to assess changes in MDMA induced memory impairments after pretreatments with $5-\mathrm{HT}_{1 \mathrm{~A}}$ and $5-\mathrm{HT}_{2 \mathrm{~A}}$ receptor blockers, the main goal of the present study.

The hypotheses that pretreatment with ketanserin or pindolol would interact with MDMA induced memory impairment was tested in two separate GLM analyses that assessed main effects of MDMA, Ketanserin and MDMA $x$ Ketanserin and main effects of MDMA, Pindolol and MDMA $x$ Pindolol respectively. The GLM models were chosen because they offer a robust and direct assessment of the interaction between pretreatments and MDMA in only 2 major analyses. This procedure was much preferred over the alternative approach of multiple comparisons between drug-drug and drug-placebo that can only provide indirect evidence of pretreatment-treatment interactions. Consequently, this study only conducted drug-placebo contrasts as supportive, secondary tests following a significant interaction between pretreatment and MDMA as evinced by GLM ANOVA.

GLM analyses revealed only one significant interaction between pretreatmenttreatment in the three memory tasks. In the WLT, pretreatment with ketanserin prevented impairment of word learning during treatment with MDMA. Separate drug-placebo contrasts also provided indirect and supportive evidence of the interaction between ketanserin and MDMA. T1-T2 combinations of placebo-MDMA and pindolol-MDMA both produced significant reductions in immediate recall. Drug-placebo contrasts also showed that performance in the word learning task after combination of ketanserin and MDMA did not 
differ from that during the placebo-placebo combination. Together these data strongly suggest that MDMA-induced impairments on verbal memory are in large part caused by direct or indirect stimulation of the $5-\mathrm{HT}_{2 \mathrm{~A}}$ receptor. This finding appears in line with a previous report on $5-\mathrm{HT}_{2 \mathrm{~A}}$ receptors densities in recent MDMA and ex-MDMA users (Reneman et al., 2002). That study showed that 5- $\mathrm{HT}_{2 \mathrm{~A}}$ receptor densities were significantly lower in recent MDMA users and significantly higher in ex-MDMA users. The authors speculated that the latter was evidence of compensatory upregulation of post-synaptic 5$\mathrm{HT}_{2 \mathrm{~A}}$ receptors due to low synaptic 5-HT levels. This study suggests that downregulation of 5$\mathrm{HT}_{2 \mathrm{~A}}$ receptors in recent MDMA users may result from high synaptic levels of 5-HT and 5$\mathrm{HT}_{2 \mathrm{~A}}$ receptor stimulation during MDMA intoxication. Studies showing involvement of $5-\mathrm{HT}_{2}$ receptors in acute MDMA intoxication as well as in persistent memory impairments in chronic MDMA users indicate that these phenomena may share related underlying mechanisms. Any definitive conclusion in that direction however remains premature as we do not know at present whether acute and chronic memory effects of MDMA are truly interconnected.

Pretreatment with pindolol did not affect memory impairments in any of the memory tasks after treatment with MDMA. The interaction between pindolol and MDMA never reached statistical significance in any of the GLM analyses. These data suggest that $5-\mathrm{HT}_{1 \mathrm{~A}}$ receptors do not play a crucial role in the neuropharmacology of MDMA induced memory impairment. These finding confirm recent results from a study assessing the role of $5-\mathrm{HT}_{1 \mathrm{~A}}$ receptors on MDMA effects on visual-spatial working memory as measured with a CANTAB test battery (Hasler et al., 2009) in a placebo-controlled study. A single dose of MDMA significant impaired visual-spatial memory as compared to placebo. However, pretreatment with pindolol did not significantly alter MDMA induced impairment of memory. It should be noted however that Pindolol is not a very selective drug. It is basically a beta-blocker that also blocks approximately $40 \%$ of $5 \mathrm{HT}_{1 \mathrm{~A}}$ receptors in the brain (Rabiner et al., 2000). Unfortunately, $5 \mathrm{HT}_{1 \mathrm{~A}}$ ligands that selectively and fully block $5 \mathrm{HT}_{1 \mathrm{~A}}$ receptors are presently not available. Nevertheless, recent molecular imaging studies also suggested that $5-\mathrm{HT}_{1 \mathrm{~A}}$ receptors may not be involved in human cognition at all (Borg, Andrée, Lundberg, Halldin, \& Farde, 2006; Borg, 2008). These studies failed to find any correlation between regional [11C]WAY 100635 binding to $5-\mathrm{HT}_{1 \mathrm{~A}}$ receptors and cognitive performance in humans as measured with standard neuropsychological tests batteries (Borg et al., 2006; Borg, 2008). 
Together, these results question the role of $5-\mathrm{HT}_{1 \mathrm{~A}}$ receptors in human memory, as it cannot explain the general memory deficit in MDMA users.

This study did not provide clues to neuropharmacological mechanisms of MDMA induced impairments in every memory domain. The preventive effect of ketanserin on MDMA induced memory impairment was selective for verbal memory performance in the Word Learning Task. Ketanserin did not affect spatial memory and prospective memory impairments following MDMA. The selectivity of the interaction between ketanserin and MDMA on verbal memory was also supported by the lack of any interactions between pretreatment and MDMA on safety measures such as blood pressure and body temperature. The latter finding is in line with a previous study that demonstrated that co-administration of ketanserin did not alter MDMA effects on physiological measures (i.e. blood pressure, heart rate and body temperature) and subjective measures (e.g. well-being and positive mood) (Herin, Liu, Ullrich, Rice, \& Cunningham, 2005; Liechti et al., 2000).

The selective interaction between ketanserin and MDMA effects on verbal memory may indicate differences in neural memory networks and differences in distributions of 5$\mathrm{HT}_{1 \mathrm{~A}}$ and $5-\mathrm{HT}_{2 \mathrm{~A}}$ receptors within these networks. It is clear from the functional neuroimaging literature that the frontal, parietal and occipitotemporal cortices are generally involved in a large variety of memory tasks, but that the pattern of brain recruitments within these networks may differ as a function of memory task or memory process (Linden, 2007). Autoradiographic mapping of $5-\mathrm{HT}_{1}$ and $5-\mathrm{HT}_{2}$ receptors in Rhesus monkeys demonstrated complementary patterns of distribution of $5-\mathrm{HT}_{1}$ and $5-\mathrm{HT}_{2}$ receptors in cortical areas of frontal, parietal and occipital lobes. The $5-\mathrm{HT}_{1}$ receptors were concentrated in layers I-III, whereas $5-\mathrm{HT}_{2}$ receptors were primarily concentrated in throughout layers III and IV (Lidow, Goldman-Rakic, Gallager, \& Rakic, 1989). Involvement of 5- $\mathrm{HT}_{2 \mathrm{~A}}$ receptors in MDMA-induced memory impairment thus may depend on task related network activations that occur within these layers.

Recent studies have also shown that a functional genetic polymorphism of the $5-\mathrm{HT}_{2 \mathrm{~A}}$ receptor (His452Tyr) has been associated with variations in memory performance, more specifically in hippocampus-dependent memory tasks (Schott et al., 2011; Sigmund, Vogler, Huynh, de Quervain, \& Papassotiropoulos, 2008). Future studies may focus on whether genetic variation of the $5-\mathrm{HT}_{2 \mathrm{~A}}$ receptors affects the magnitude of MDMA induced verbal 
86 IChapter 5

memory impairment or determines the sensitivity of MDMA users for MDMA induced impairment of verbal memory.

In sum, this study demonstrated that single doses of MDMA impaired memory in a range of tasks, and that pretreatment with ketanserin offered selective protection against MDMA induced verbal memory impairment. This demonstrates that MDMA induced verbal memory impairment is mediated by $5-\mathrm{HT}_{2 \mathrm{~A}}$ receptor stimulation. 
$88 \mid$ 


\section{CHAPTER 6}

\section{EFFECTS OF ACUTE MDMA INTOXICATION ON MOOD AND} IMPULSIVITY: ROLE OF THE 5-HT ${ }_{2}$ AND 5-HT ${ }_{1}$ RECEPTORS 


\section{Summary}

MDMA induces positive mood and increases impulse control during intoxication, but only a few studies on the neuropharmacological mechanisms underlying these processes have been conducted. It was hypothesized that pretreatment with $5-\mathrm{HT}_{1}$ and $5-\mathrm{HT}_{2}$ receptor blockers would prevent MDMA effects on mood and impulsivity. Subjects $(N=17)$ participated in a double-blind, placebo controlled, within-subject design involving 6 experimental conditions consisting of pretreatment ( $\mathrm{T} 1)$ and treatment (T2). T1 preceded T2 by 30 minutes. T1-T2 combinations were: placebo-placebo, $20 \mathrm{mg}$ pindolol-placebo, $50 \mathrm{mg}$ ketanserin-placebo, placebo-75mg MDMA, 20mg pindolol-75mg MDMA and 50mg ketanserin-75g MDMA. Subjects completed a Profile of Mood States (POMS) questionnaire and several impulsivity tasks (Stop signal task, Matching familiar figures task, Cue dependent reversal learning task) at $1.5 \mathrm{hrs}$ post-treatment.

MDMA alone increased both positive (vigor, arousal, friendliness, elation, positive mood) and negative affect (anxiety, confusion) as assessed by the POMS questionnaire. MDMA also increased stop reaction time in the Stop signal task and reaction time in the Matching familiar figures task. Pretreatment with ketanserin blocked MDMA effects on positive affect, but not negative affect. Ketanserin did not influence the effects of MDMA on impulsivity. Pindolol did not interact with MDMA on any of the measures. In conclusion, 5$\mathrm{HT}_{2}$ receptors mediate positive moods induced by MDMA but not negative moods or impulsivity. $5-\mathrm{HT}_{1}$ receptors do not appear to be involved in MDMA effects on mood and impulse control. 


\section{Introduction}

3,4-Methylenedioxymethamphetamine (MDMA) is a serotonin (5-HT) agonist and a reuptake inhibitor of serotonin and dopamine (DA) that has been shown to affect mood (Bosker et al., 2010) and impulsivity during intoxication (Kuypers et al., 2007; Ramaekers \& Kuypers, 2006; Vollenweider et al., 2001) and abstinence (Curran \& Travill, 1997; Morgan, 1998). Mood has been shown to be affected by fluctuations in 5-HT levels. High levels of 5-HT have been associated with mood elevations, while decrements in 5-HT are associated with depressed mood (Flory, Manuck, Matthews, \& Muldoon, 2004; Toker, Amar, Bersudsky, Benjamin, \& Klein, 2010; Young \& Leyton, 2002). MDMA has been shown to increase 5-HT levels during intoxication but to deplete 5-HT after intake (Flory et al., 2004; Parrott, 2012; Schmidt, 1987; Toker et al., 2010). Vollenweider and colleagues (2001) discuss a series of studies in which a single dose of MDMA was shown to directly increase subjective feelings of openness, enhance mood and well-being and heighten feelings of happiness. Other researchers also reported that MDMA increased subjective ratings of feeling 'loving' and 'friendly', as indicated by the Profile of Mood States (POMS) questionnaire (Bedi, Hyman, \& de Wit, 2010). However, depressed mood has been reported in MDMA-users following acute administration of the drug, probably due to a depletion of 5-HT stores (Parrott \& Lasky, 1998; Parrott, Lock, Conner, Kissling, \& Thome, 2008; Verheyden, Hadfield, Calin, \& Curran, 2002).

A similar relation may hold true for MDMA effects on impulsive behaviors. A reduction of 5-HT has been linked to impulsive, suicidal and aggressive behaviour (Fitzgerald, 2011) and substance abuse (Kirby et al., 2011). In contrast, high levels of 5-HT have been shown to decrease impulsive behaviour (Fitzgerald, 2011; Pattij \& Vanderschuren, 2008). Likewise, acute MDMA administration has been shown to increase impulse control, when 5HT levels are high (Bosker et al., 2010; Kuypers et al., 2006, 2007; Ramaekers \& Kuypers, 2006), whereas impulse control decreased in the period following use, when 5-HT levels are low (Butler \& Montgomery, 2004; Gouzoulis-Mayfrank \& Daumann, 2009; Morgan, 1998; Morgan, Impallomeni, Pirona, \& Rogers, 2006; Piechatzek et al., 2009; Quednow et al., 2007; Reneman et al., 2002).

Besides general 5- $\mathrm{HT}$ levels, there is also evidence that $5-\mathrm{HT}_{1}$ and $5-\mathrm{HT}_{2}$ receptors contribute to the influence of MDMA on mood and impulsivity (Liechti et al., 2000). Liechti and Vollenweider (2001) have shown that a $5-\mathrm{HT}_{2}$ receptor antagonist (ketanserin) 
decreased the effects of MDMA on perception and emotional excitation. These investigators also demonstrated that a $5-\mathrm{HT}_{1}$ blocker (pindolol) ameliorated some, but not all, of the MDMA-induced subjective effects (Vollenweider, Studerus, Hasler, \& Paulus, 2006). The role of $5-\mathrm{HT}_{1}$ and $5-\mathrm{HT}_{2}$ antagonists on MDMA effects in impulsivity has not been studied before. However it has been shown that these receptors may play a role in impulse control. For example, 5- $\mathrm{HT}_{2}$ antagonists have been shown to decrease impulsivity (Anastasio et al., 2011) whereas $5-\mathrm{HT}_{1}$ agonists have been associated with reductions in anxiety and impulsivity (Schreiber \& de Vry, 1993).

The present study was designed to assess the role of $5-\mathrm{HT}_{1}$ and $5-\mathrm{HT}_{2}$ receptors on the effects of MDMA on mood and impulse control. It was hypothesized that (1) an acute dose of MDMA would alter mood and increase impulse control; and that (2) the effects of MDMA on mood and impulsivity would be absent when pre-treated with $5-\mathrm{HT}_{1}$ and $5-\mathrm{HT}_{2}$ receptor blockers.

\section{Materials and methods}

\section{Subjects}

Seventeen healthy MDMA-users (9 male, 8 female), aged between 19 and 27 (mean (SD) $22.76(2.75))$ years participated in the study. They were mild to moderate users of MDMA who reported to have taken the drug on 1 to 65 separate occasions (mean 10.94) in the previous year. Overall, subjects reported to have taken MDMA 3 to 780 occasions in their lifetime (mean 72.4 times). Subjects reported mean lifetime use of alcohol on 1168.5 occasions; cannabis on 554.4 occasions; amphetamines on 4.1 occasions; cocaine on 4.7 occasions; LSD on 4.4 occasions; mushrooms on 0.2 occasions; and other drugs, including but not limited to, khat, ketamine or salvia divinorum, 0.5 occasions.

Subjects were recruited through advertisements in local newspapers and by word of mouth. Before inclusion, subjects were examined by a medical supervisor, who checked vital signs and took blood samples for standard blood chemistry and haematology. Inclusion criteria were: written informed consent; age 18-35 years; history of MDMA use; free from psychotropic medication; good physical health as assessed by a medical doctor; normal weight as determined by BMI 18-28. Exclusion criteria were: addiction according to DSM-IV criteria as assessed by a questionnaire; presence or history of psychiatric or neurological disorder as assessed during a clinical interview; pregnancy or lactating; cardiovascular 
abnormalities; excessive drinking or heavy smoking, i.e. defined as more than 20 standard units of alcohol per week and more than 10 cigarettes per day; hypertension. Subjects were given an information leaflet before the study explaining the entire study procedure. Subjects were fully aware of everything that would happen during the study, except the order of treatment.

This study was conducted according to the code of ethics on human experimentation established by the declaration of Helsinki (1964) and amended in Seoul (2008) and was approved by the Medical Ethics Committee of the Academic Hospital of Maastricht and Maastricht University. A permit for obtaining, storing and administering MDMA was obtained from the Dutch drug enforcement administration. Subjects were paid for their participation in the study.

\section{Design, doses and administration}

Subjects participated in a double-blind, placebo controlled, within-subject design involving 6 experimental conditions consisting of pretreatment (T1) and treatment (T2). T1 preceded T2 by 30 minutes. T1-T2 combinations were: placebo-placebo, $20 \mathrm{mg}$ pindolol-placebo, $50 \mathrm{mg}$ ketanserin-placebo, placebo-75 mg MDMA, pindolol $20 \mathrm{mg}-\mathrm{MDMA} 75 \mathrm{mg}$ and $50 \mathrm{mg}$ ketanserin-75 mg MDMA. Conditions were separated by minimum wash-out period of 7 days to avoid cross-condition contamination. Order of conditions was divided in 3 blocks of 6 conditions. The $75 \mathrm{mg}$ dose of (racemic) MDMA was selected because it falls within the normal range of recreational use (Gouzoulis-Mayfrank \& Daumann, 2009) and has been consistently shown to impair performance and produce robust subjective mood changes in a number of previous studies from our group (Kuypers \& Ramaekers, 2005, 2007; Ramaekers, Kuypers, et al., 2009). Doses of pindolol $20 \mathrm{mg}$ and ketanserin $50 \mathrm{mg}$ represent regular therapeutic doses that block approximately $40 \%$ of $5-\mathrm{HT}_{1 \mathrm{~A}}$ receptors and $91 \%$ of $5-\mathrm{HT}_{2}$ receptors respectively (Brogden \& Sorkin, 1990; Rabiner et al., 2000; Sharpley et al., 1994). MDMA, pindolol and ketanserin were acquired through the local hospital pharmacy, which also performed randomization, capsulation and distribution of study drugs.

\section{Procedures}

All subjects received a training session before onset of the experimental sessions in order to familiarize them with the tests and procedures. Subjects were asked to refrain from drugs at 
least a week before the start of the experiment and during the study. Subjects were not allowed to use alcohol on the day prior to an experimental session and were requested to arrive at experimental sessions well rested. Drug and alcohol screens were performed upon arrival of the subject. Drug screens assessed the presence of benzodiazepines, opiates, cocaine, marijuana, MDMA and (meth)amphetamine. A pregnancy test was performed for the female subjects. Study treatments were only administered if subjects tested negative for drugs, alcohol and pregnancy.

Treatments at T1 and T2 were administered using a double dummy technique to synchronize time of maximal drug concentrations $\left(T_{\max }\right)$ and were administered as identical encapsulated tablets to ensure blinding. Mood and impulsivity were assessed by means of a number of tasks between 1.5-2 hrs after T2 (at $\mathrm{T}_{\max }$ ). In between, subjects were allowed to

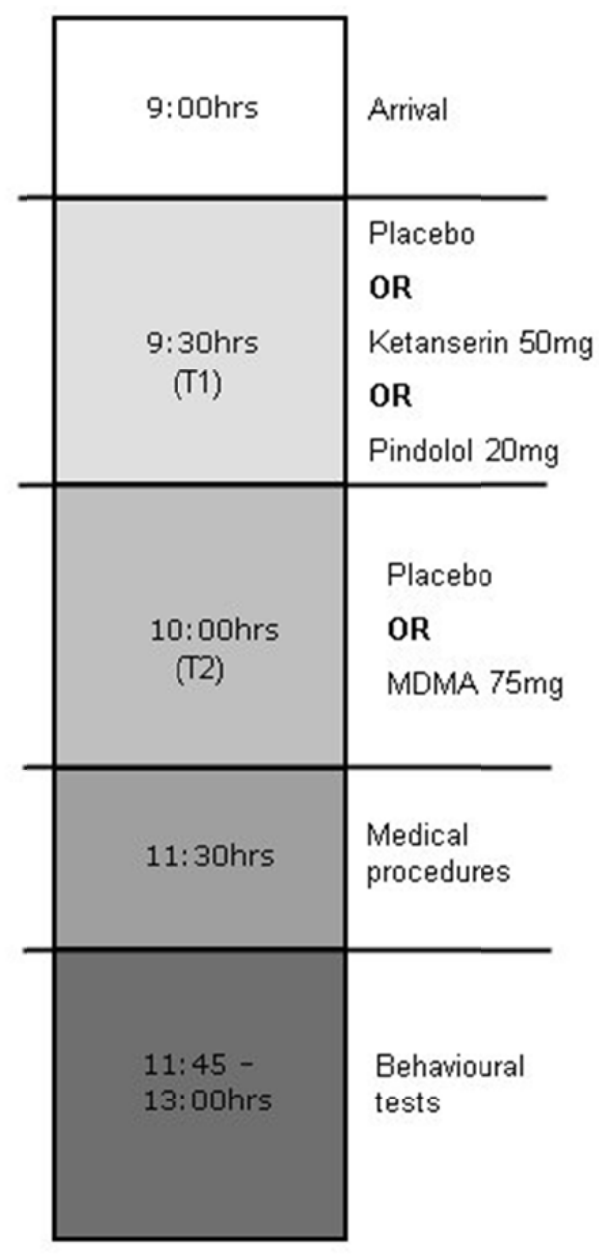
read a book or watch television. In addition, blood pressure and body temperature were assessed as safety measures (more details about physiological measures are described in a previous paper by van Wel et al. (2011). A schematic representation of a testing day is shown in figure 1 . The results described in this paper are part of a larger study described elsewhere in this thesis.

\section{Subjective measures \\ Profile of Mood States \\ The Profile of Mood States (POMS) is a \\ self-assessment mood questionnaire with 72 items, rated on a 5-point Likert scale, with 0 being 'not at all' to 4 'extremely'. Subjects had to indicate to what extent these items were}

Figure 1: Schematic representation of a testing day. 
representative of their mood at that moment in time. Eight mood states are classified and quantified by calculating the sum score of associated items for each mood state, i.e., anxiety (9 items), depression (15 items), anger (12 items), vigor (8 items), fatigue (7 items), confusion (7 items), friendliness ( 8 items) and elation ( 6 items). Two composite scales were derived; arousal ((anxiety+vigor)-(fatigue+confusion)) and positive mood (elation-depression) (de Wit et al., 2002).

\section{Impulsivity tests}

\section{Matching Familiar Figures Task}

The computerized version of the Matching Familiar Figures Test (MFF20) was derived from the original Matching Familiar Figures Test (Kagan, 1966) by Cairns and Cammock (1978) and serves as a measure of reflection impulsivity. This is the tendency to reflect on the validity of a problem to be solved under the specific condition that there are more alternatives available while there is some uncertainty over which is the right choice. Thus, subjects who tend to respond more impulsively are faster, but make more errors, while those who pause and think about the alternatives are slower and more accurate. This task was developed to assess the processes involved in the gathering and evaluation of perceptual information required to make a response. The MFF20 seems to share some variability with other impulsivity tasks, such as the stop-signal task, but not with tasks involving reward (Perales, Verdejo-Garcia, Moya, Lozano, \& Perez-Garcia, 2009b).

The test format of the computerized MFF20 involves simultaneous presentation of a target figure positioned on the left of the screen and an array of six alternatives on the right half of the screen, all except one differing in one or more details from the target figure. The subjects are asked to select from the alternatives the figure that exactly matches the target as quickly as possible. This is achieved by pressing the number corresponding to the figure on a computer keyboard. If the initial selection is incorrect, this is signaled with a beep and subjects are required to give another answer. Each subject is given 2 practice trials followed by 20 test trials.

Two dependent measures, mean latency to first response and total number of errors, are automatically recorded. Two additional dependent variables can be calculated: an Impulsivity score (I-score) and an Efficiency score (E-score). The I-score is a composite index of impulsivity (Messer \& Brodzinsky, 1981; Salkind \& Wright, 1977) and is calculated by 
subtracting the standard score of the mean latency to first response from the standard score of the total number of errors committed (Zerror-Zlatency). The E-score is calculated by summing the standard score of the mean latency to first response with the standard score of the total number of errors committed (1-(Zerror+Zlatency)).

\section{$\underline{\text { Stop signal task }}$}

The stop signal task provides a measure of motor impulsivity. It requires subjects to make quick responses to visually presented go signals and to inhibit their response when a visual stop signal is suddenly presented. The current test is adapted from an earlier version of Fillmore and colleagues (2002) and has been shown to be sensitive to stimulating as well as sedating drugs (Ramaekers \& Kuypers, 2006). The go signals are four $1.5 \mathrm{~cm}$ letters (ABCD) presented one at a time in the center of a computer screen. Subjects are required to respond to each letter as quickly as possible by pressing one of two response buttons. One button is pressed to indicate that " $A$ " or " $C$ " appeared and the other to indicate " $B$ " or " $D$ ". Letters are displayed for 500 milliseconds and the computer screen is blank for 1.5 seconds before the next letter is displayed. This provides a period of 2 seconds in which the subject can respond to a letter. A single test consists of 176 trials in which each of the 4 stimuli is presented equally often. A stop signal occurs in 48 trials during the test. The stop signal consists of a visual cue, i.e. "*", that appears in one of the four corners of the screen. Subjects are required to withhold their response in case a stop signal is presented. Stop signals are presented 12 times at each of the four delays after the onset of a letter: 50,150 , 250 and 350 milliseconds. Trials always begin with a 500 millisecond preparation interval in which a fixation cross appears at the center of the screen. Dependent variables are the proportion of commission errors on stop signal trials and the reaction times on go and stop signal trials (i.e. stop reaction time). Stop reaction time (SRT) to stop signal trials represents the estimated mean time required to inhibit a response.

The method for calculating stop reaction time was taken from the race model of inhibitory control (Logan \& Cowan, 1984). This model proposes that the response to stop signal trials is defined by two parallel processes: execution of a motor action in response to a signal and inhibition of a motor action in response to a stop signal. Crucial to the outcome of the race is the speed of both processes. Response inhibition will fail if the time required to inhibit exceeds the time to complete a motor response at the time of the stop signal. The 
speed of the inhibition response cannot be observed directly but can be derived mathematically on the basis of three factors: stop-signal delay, reaction time distribution on go trials and the probability of successful response inhibitions in stop signal trials. First, reaction times to 128 go trials were rank ordered from shortest to longest. The finishing time of the inhibition response was then determined from the probability of successful response inhibition and the distribution of reaction times. If $n$-percent of the responses on stop-signal trials would be unsuccessfully inhibited (i.e. commission error), than the finishing time would be associated with the $n$-th percentile of the RT distribution. Stop reaction time was then determined by subtracting the appropriate stop-signal delay from reaction time at the $n$-th percentile of the RT distribution. The resulting values for each stop signal delay were then averaged to yield a single measure of stop reaction time for the test (Ramaekers \& Kuypers, 2006).

\section{Cue-dependent reversal learning task}

The cue-dependent reversal learning task is an adjusted form of the cue-dependent go-nogo task of Fillmore and Rush (2006). This test places emphasis on the anticipatory nature of inhibitory and activational mechanisms of control, which rapidly develop cue-dependence. It has also been shown to be highly sensitive to the effects of psychoactive drugs (Fillmore et al., 2006). In this task, subjects are required to respond to target stimuli (Go) and to inhibit their response on non-target stimuli (No-Go). Stimuli are rectangles, appearing in the center of a computer screen, in a horizontal or vertical position. Targets are green and non-targets are blue rectangles. Cues provide preliminary information regarding the type of imperative target stimulus (i.e. Go or No-Go) that is likely to follow. The cues have a high probability of signaling the correct target. The 'vertical rectangle' precedes a Go-stimulus in $80 \%$ of the cases and a No-Go-stimulus in $20 \%$ of the cases. The 'horizontal rectangle' signals a No-Go in $80 \%$ of the cases and a Go-stimulus in $20 \%$ of the cases. This rule will be reversed a number of times throughout this task, depending on the performance of the subject. Subjects have to detect this rule-change and modify their response pattern. Subjects will be informed beforehand that the rule can change but not about the number of times this event will take place or when it is changed. This task measures the acquisition and discrimination-reversal learning of cue-target associations. More impulsive individuals have more problems 
inhibiting responses after a reversal of the rule than less impulsive individuals (Fillmore et al., 2006). Dependent variables are number of correct responses and correct inhibitions.

\section{Blood samples}

Blood samples were collected before the start of the tasks at $1.5 \mathrm{hrs}$ post T2. Blood samples were centrifuged immediately and the serum was subsequently frozen at $-20^{\circ} \mathrm{C}$ until analyses for pharmacokinetic assessments. MDMA, pindolol and ketanserin concentrations were determined using solid phase extraction and gas chromatography with mass spectrometric detection.

\section{Statistics}

The hypothesis that pretreatment with ketanserin or pindolol would interact with MDMA induced impulsivity and mood was tested in 2 separate General Linear Model (GLM) analyses. Impulsivity and mood effects of MDMA, Ketanserin and MDMA x Ketanserin were analyzed by means of a GLM repeated measures ANOVA with MDMA (2 levels, i.e. $75 \mathrm{mg}$ MDMA and placebo) and Ketanserin (50 mg ketanserin and $50 \mathrm{mg}$ ketanserin $+75 \mathrm{mg}$ MDMA) as the main factors. Impulsivity and mood effects of MDMA, Pindolol and MDMA $x$ Pindolol were analyzed by means of a GLM repeated measures ANOVA with MDMA (2 levels, i.e. $75 \mathrm{mg}$ MDMA and placebo) and Pindolol (20 mg pindolol and $20 \mathrm{mg}$ pindolol $+75 \mathrm{mg}$ MDMA) as the main factors. In case of significant main effects, separate drug-placebo contrasts were conducted. The alpha criterion significance level was set at $p=0.05$. All statistical tests were conducted with SPSS version 15.0.

\section{Results}

Seventeen complete data sets entered statistical analysis, except for the Stop signal task $(\mathrm{N}=14)$, where 3 subjects were excluded due to technical or performance failures during test administration.

\section{Subjective measures}

POMS

A summary of GLM statistics for all POMS scales is given in Table 1. Mean (SE) subjective ratings on the POMS scales in every treatment condition are shown in Figure 2. 
MDMA significantly increased feelings of anxiety, confusion, vigor, friendliness, elation, positive mood and arousal. The effect of MDMA on positive mood was highly significant $(p<.001)$ in the GLM model including pindolol, and almost reached significance $(p=0.057)$ in the GLM model including ketanserin. In addition, MDMA also significantly reduced feelings of fatigue. Pindolol significantly increased feelings of confusion and decreased positive mood. There was no significant interaction between pindolol and MDMA. Ketanserin
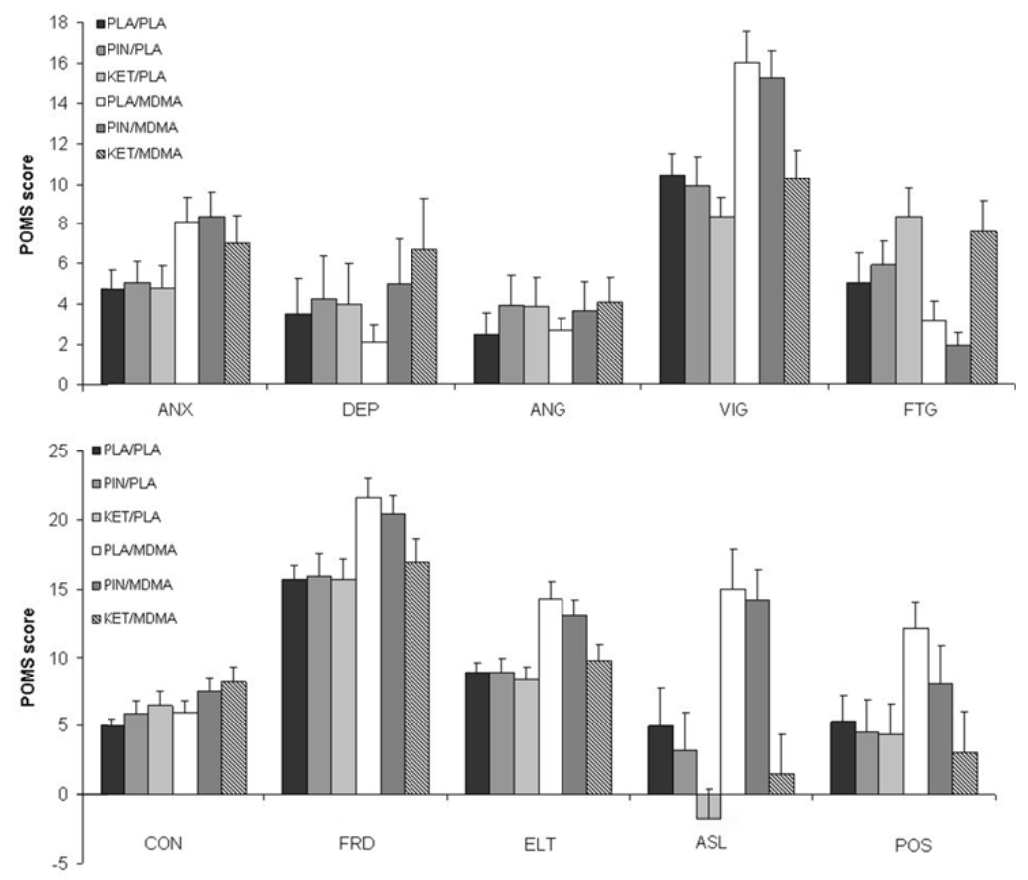

Figure 2: Mean (SE) ratings on the Profile of Mood States (POMS) questionnaire in every treatment condition $(n=17)$. ANX = anxiety, DEP = depression, ANG = anger, VIG = vigor, $\mathrm{FTG}=$ fatigue, $\mathrm{CON}=$ confusion, $\mathrm{FRD}=$ friendliness, $\mathrm{ELT}=$ elation, $\mathrm{ASL}=$ arousal, POS = positive mood.

affected almost all subscales of the POMS. It significantly increased feelings of depression, fatigue and confusion and decreased feelings of vigor, friendliness, elation, arousal and positive mood. The interaction between MDMA and ketanserin reached significance on 4 POMS subscales: depression, friendliness, elation and positive mood. After the combination of MDMA and ketanserin, ratings of friendliness, elation and positive mood returned to placebo levels, whereas feelings of depression increased. 


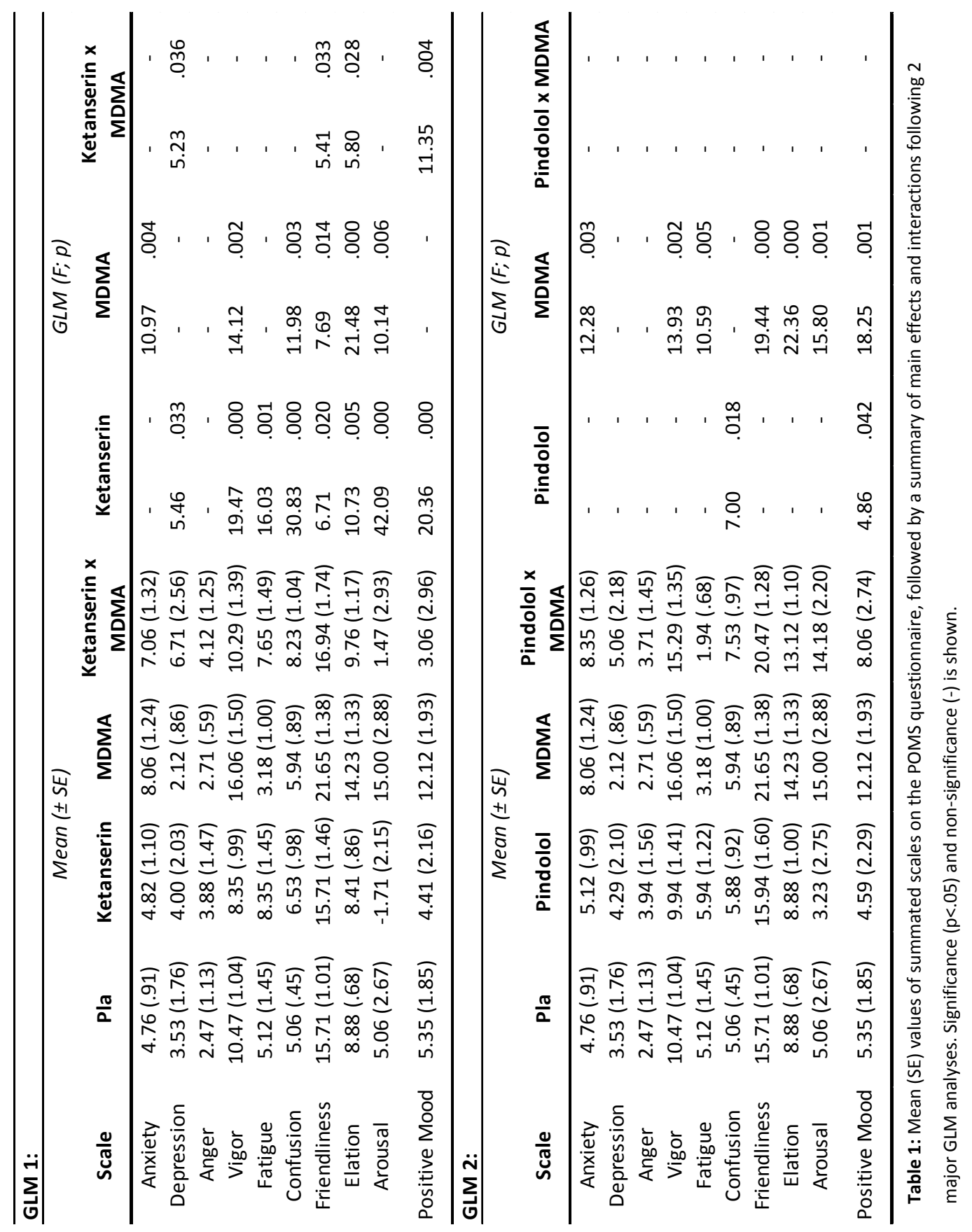




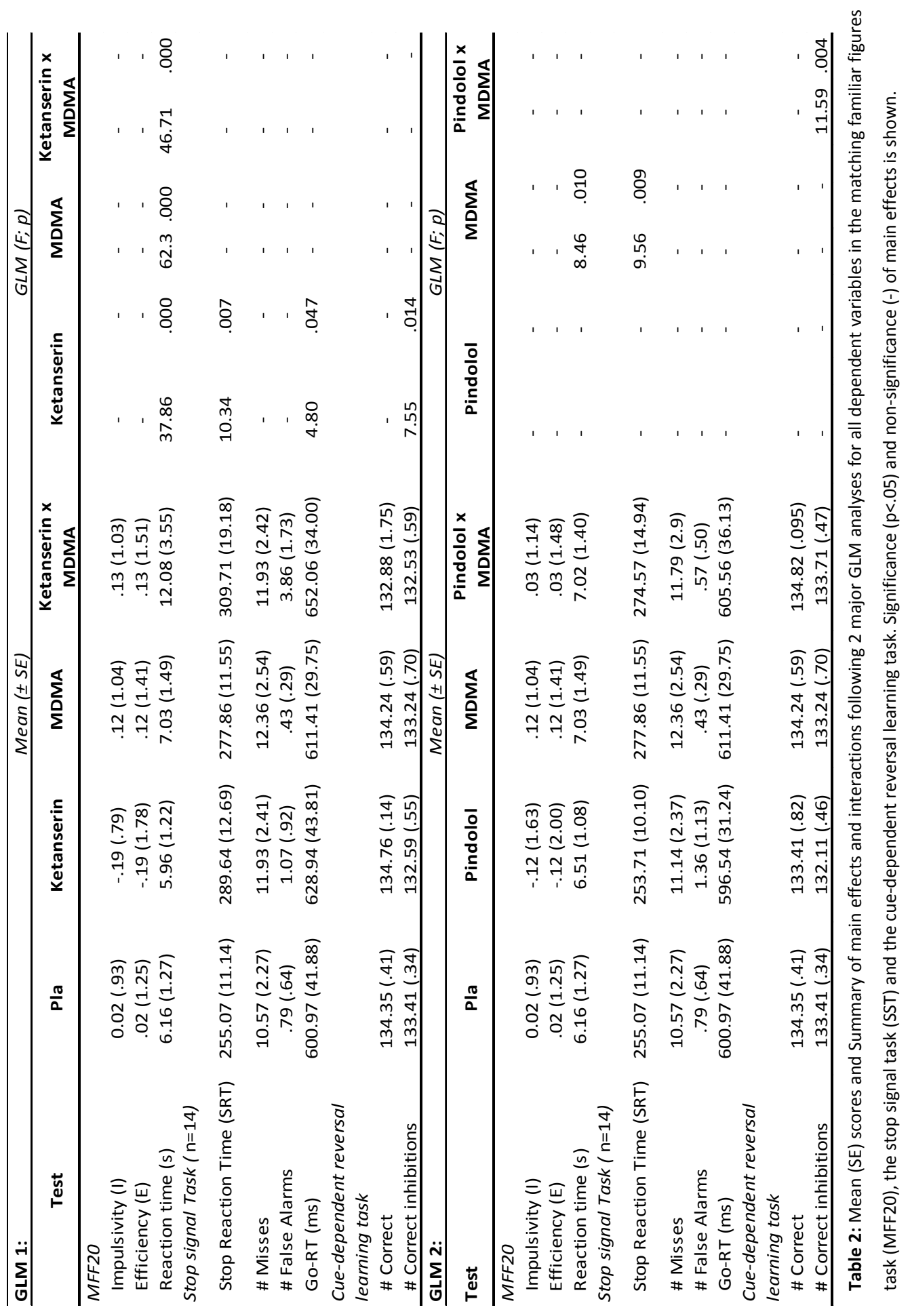


Impulsivity tasks

The mean (SE) performance on impulsivity tasks for every treatment condition and a summary of GLM statistics for all impulsivity parameters is given in Table 2.

MDMA significantly increased SRT in the stop signal task and RT in the MFF20 but generally failed to affect performance on any of the other impulsivity parameters. Pindolol did not affect any impulsivity parameters. Ketanserin increased SRT and RT in the stop signal task and decreased the number of inhibitions in the cue-dependent reversal learning task. There were no significant interactions between MDMA and ketanserin. There were also no interactions between MDMA and pindolol other than an increase in correct inhibitions in the cue-dependent reversal learning task.

\section{Blood samples}

Mean (SD) MDMA concentration in serum at 1.5 hours after MDMA administration was 157 (48) $\mathrm{ng} / \mathrm{mL}$. When combined with ketanserin or pindolol, mean (SD) serum concentration was 164 (62) and 156 (56) ng/mL respectively. Mean (SD) serum concentrations of ketanserin and pindolol were $86(42)$ and 133 (80) ng/mL respectively. In the condition where ketanserin or pindolol was combined with MDMA, the mean (SD) serum concentrations of both drugs were 104 (41) and 130 (53) ng/mL respectively.

\section{Discussion}

The aim of the current study was to investigate the role of $5-\mathrm{HT}_{1}$ and $5-\mathrm{HT}_{2}$ receptors in MDMA induced changes in mood and impulsive behaviour. Single doses of MDMA significantly increased positive as well as negative moods as rated with the POMS questionnaire. MDMA raised feelings of vigour, friendliness, elation and arousal, while also making subjects feel more anxious and confused. These findings are in line with previous studies that also reported a marked effect, both positive and negative, of MDMA administration on mood ratings (Bedi et al., 2010; Bosker et al., 2010; Kuypers et al., 2006, 2007; Liechti et al., 2000; Liechti, Baumann, Gamma, \& Vollenweider, 1998; Parrott \& Stuart, 1997).

Single doses of MDMA increased SRT in a stop signal task and reaction time in the MFF20 indicating a slowing of inhibitory and reflective responses during these tasks. Other measures of impulsivity did not show any effects of MDMA. 
In general however, acute effects of MDMA on mood and impulsivity were sufficiently present to assess the contributing roles of $5-\mathrm{HT}_{1}$ and $5-\mathrm{HT}_{2}$ receptors during pretreatments with ketanserin and pindolol.

Pretreatment with ketanserin significantly interacted with MDMA on the subscales representing positive moods (friendliness, elation and positive mood). Blockade of 5- $\mathrm{HT}_{2}$ receptors with ketanserin basically prevented MDMA to affect positive moods at all. POMS ratings of positive mood during the combination of ketanserin and MDMA were similar to the ratings during placebo. Ketanserin alone also significantly decreased positive mood rating. The magnitude of these effects was very small relative to the increase in positive moods produced by MDMA. Consequently, combined effects of ketanserin and MDMA cannot be explained as a summation of drug effects produced by MDMA and ketanserin separately, but truly indicates a drug interaction indicating that blockade of $5-\mathrm{HT}_{2}$ receptors also blocks MDMA effects on positive moods. Pretreatment with ketanserin however did not reverse MDMA induced anxiety and the combination increased ratings of depression. This finding strongly indicates that the $5-\mathrm{HT}_{2}$ receptor is only involved in mediating positive moods during intoxication and is not involved in some of the negative moods produced by MDMA.

Pretreatment with pindolol did not interact with the effects of MDMA on mood. When given alone, pindolol produced small but significant increments in feelings of confusion and small decrements in positive mood. The lack of interaction between MDMA and pindolol illustrated that the $5-\mathrm{HT}_{1}$ receptor does not play a role in mediating MDMA induced mood states. The finding is in line with a previous mechanistic study (Hasler et al., 2009) showing that pretreatment with pindolol does not affect MDMA induced moods. Alternatively, one could also argue that pindolol blocks only $40 \%$ of $5 \mathrm{HT}_{1 \mathrm{~A}}$ receptors (Brogden \& Sorkin, 1990) and that this may not suffice to measurably attenuate any $5 \mathrm{HT}_{1 \mathrm{~A}}$ mediated MDMA effects. We cannot exclude this possibility, but unfortunately, alternative $5 \mathrm{HT}_{1 \mathrm{~A}}$ ligands that fully block $5 \mathrm{HT}_{1 \mathrm{~A}}$ receptors are presently not available.

None of the pretreatments interacted with the effects of MDMA on measures of impulsivity. It should be noted, however, that the effects of MDMA were limited to an increment of SRT in the stop signal task and reaction time in the MFF20 and did not affect other measures of impulsivity. Previous studies have also shown a mix of either positive or 
neutral effects of single doses of MDMA on impulsivity (Bosker et al., 2010; Fitzgerald, 2011; Kuypers et al., 2006, 2007; Pattij \& Vanderschuren, 2008). Possibly, the lack of MDMA effects on most measures of impulsivity may be related to the fact that these measures represent different psychological and neuropharmacological constructs of impulse control (Talpos, Wilkinson, \& Robbins, 2006). Impulsivity is not a unitary, one-dimensional construct but can encompass different types of impulsivity (Chambers et al., 2009; Dalley et al., 2011; Evenden, 1999). Two types of impulsivity that can be distinguished are cognitive impulsivity and motor impulsivity. Cognitive impulsivity, as measured by the MFF20, is believed to reflect complex processes involved in the control of several cognitive, behavioral and effective processes. Motor impulsivity or response inhibition as measured by the stop signal task and the cue dependent reversal learning task, on the other hand, is believed to relate to the executive control of motor processes only (Friedman \& Miyake, 2004). Thus, it is possible that MDMA affects only a subset of processes related to motor impulsivity and cognitive impulsivity but leaves other subsets unaffected (Ramaekers \& Kuypers, 2006). Alternatively, the relationship between MDMA and impulsivity may also be marginal and not a key feature during MDMA intoxication.

In conclusion, results from the current study show that administration of MDMA has both positive and negative influences on mood states. Furthermore, pretreatment with a 5$\mathrm{HT}_{2}$ receptor antagonist affects MDMA-mediated responses on a number of positive subscales of the POMS, suggesting that the $5-\mathrm{HT}_{2}$ receptor might be involved in mediating positive mood states. On the contrary, treatment with a partial $5-\mathrm{HT}_{1}$ receptor antagonist did not interfere with MDMA effects on mood. Blockade of $5-\mathrm{HT}_{1}$ and $5-\mathrm{HT}_{2}$ receptors did not interact with the effects of MDMA on measures of impulse control. 


\section{Chapter 7}

GENERAL DISCUSSION 


\section{GENERAL DISCUSSION}

The main aim of the present thesis was to investigate the effects of cannabis, cocaine and MDMA on impulsivity, cognition and mood. In this discussion I will provide an overview of the main findings and then discuss these in a broader context.

\section{Impulsivity, drugs of abuse and addiction}

The effects of cannabis, cocaine and MDMA on measures of impulsivity are described in chapters 2, 4 and 6. Results of the studies indicated that measures of impulse control were not affected during MDMA intoxication while cannabis and cocaine decreased impulse control, but in opposing ways.

The study described in chapter 6 showed that while MDMA increased reaction time on measures of motor and cognitive impulsivity, subjects did not make more errors. This indicates that MDMA does not influence impulse control per se, although the increased reaction time might reflect a compensation mechanism during MDMA intoxication where subjects need to take more time to reflect on their responses in order to choose the right one. Single doses of cannabis were shown to increase reaction time on a cognitive impulsivity task and reward anticipation task, to increase the number of errors in cognitive and motor impulsivity tasks and to decrease the accuracy in the reward anticipation task (chapter 2 and 4). This implies that cannabis intoxication negatively influences response selection as evinced by the increase in errors even when taking more time to respond. Cocaine administration increased the number of errors and decreased reaction times in the motor impulsivity task, while the drug improved performance on tasks measuring cognitive impulsivity and reward anticipation. This suggests that under the influence of cocaine, motor control over impulsive responding is diminished. This becomes a problem when a quick alteration of the response is needed, such as in the motor impulsivity task where subjects have to stop a response that was already initiated. However, when subjects are not under time pressure to decide on their response, like in the cognitive impulsivity task, or if they only have to respond quickly but not have to alter their response, such as in the reward sensitivity task, cocaine is actually beneficial.

It has been suggested that there are two mechanisms through which impulsive responding is controlled. These systems are a reflective pathway based in the prefrontal 
cortex and an impulsive pathway originating from the striatum (Bechara, 2005). If the prefrontal cortex does not function properly, explicit control over behaviour diminishes which is characterized by impairment in cognitive impulsivity. On the other hand, dopaminergic hyperactivity in the striatum is responsible for generating more impulses and motor responses which leads to impaired response inhibition and an increased sensitivity to reward. Our results indicate that cannabis administration mainly decreases the effectiveness of the reflective system, since subjects show impairments in decision making and reward anticipation. Cocaine ingestion seems to increase impulses generated from the striatum, since under influence, subjects are more sensitive to reward and tend to respond faster.

Further evidence for the existence of two control mechanisms comes from recent research (Baker, Stockwell, Barnes, \& Holroyd, 2011), indicating that two types of addicts can be distinguished: i.e. those less sensitive to rewards and those less sensitive to errors. The former type could be characterized by an altered impulsive system, since reward sensitivity is mediated by the striatum (Haber \& Knutson, 2010). The latter would be due to a disturbed reflective system, as it has been shown that decreased error sensitivity is connected to impaired top-down regulation by the dorsolateral prefrontal cortex (dIPFC) (Sokhadze et al., 2012). Eventually, knowing which mechanism or brain area is affected by which drug could advance treatment options for addiction. Currently, the treatment of addiction has mainly focused on detoxification and relapse prevention, but such programmes are only effective in about 40-60\% of all help-seeking addicts (McLellan, Lewis, O'Brien, \& Kleber, 2000). Based on our results it could be suggested that addiction should be treated differently according to the problems exhibited by impaired reflective or impulsive responding. One option for treating patients who exhibit increased impulsive responding is the use of GABA agonists like baclofen and vigabatrin. These drugs inhibit dopaminergic firing in the limbic system, which decreases excitatory $D_{1}$ receptor activity and this, in turn, increases inhibitory $D_{2}$ receptor activity (Cohen \& Frank, 2009). By this mechanism, impulsive responding is decreased while top-down regulation of behaviour by the dIPFC is improved. GABA agonist drugs have been applied in both preclinical and clinical settings, and have shown efficacy in reducing drug seeking and consumption in a number of substances such as alcohol, cocaine and nicotine (Lingford-Hughes, Watson, Kalk, \& Reid, 2010). On the other hand, reinstatement of drug seeking has been associated with increases in glutamatergic transmission in the prefrontal cortex (McFarland, Lapish, \& Kalivas, 2003). Therefore, in 
addicts that have reduced reflective capacity, administration of glutamate antagonists might be a viable treatment.

\section{Brain activity and reward during drug intoxication}

As described in chapter 4, a task designed to activate reward mechanisms in the striatum and thalamus was administered during cannabis or cocaine intoxication (the Monetary Incentive Delay Task, MIDT). A decrease in BOLD response in the thalamus was seen following both cocaine and cannabis administration relative to placebo. Paradoxically, on the behavioral level the results were orthogonal in that cannabis decreased performance (reflected by increased reaction times and less hits) while cocaine had the opposite effect. The cannabis-induced performance impairment and the cocaine-induced performance enhancement were significantly correlated with thalamus deactivation. The thalamus is an important relay station in the brain and functions as a gatekeeper for stimuli by deciding whether or not a reaction has to be executed. There are three pathways associated with thalamic control over behavior, the hyperdirect, direct and indirect pathway. After an incoming stimulus, the hyperdirect pathway initially suppresses all motor responses by inhibiting thalamic output to the cortex. Following this, the direct or indirect pathways are free to respectively execute or inhibit responding. Both the direct and indirect pathways exert their effects on the thalamus through the striatum. However, while the first pathway directly affects the thalamus through the globus pallidus interna, the indirect pathway first passes through the globus pallidus externa after which it follows the same path (Nambu et al., 2002). Increased striatal dopaminergic tone activates the direct pathway, while a decrease in dopamine levels causes more activity of the indirect pathway (Frank, 2005). During placebo, a BOLD increase of the thalamus due to activation of the direct pathway was expected since subjects always had to respond following a cue. Following from our results, it is possible that after the initial inhibition of the thalamus by the hyperdirect pathway, cocaine increased activity in the direct pathway, while cannabis increased activity in the indirect pathway due to the different effects of both drugs on the dopamine level in the brain. Cocaine has been shown to increase dopamine (Bonci et al., 2003), while the acute effects of cannabis are less clear. However, administration of cannabis to heavy cannabis users, like in our study, has been associated with decreased reactivity of dopaminergic neurons (Urban, Slifstein, Thompson, et al., 2012). Hereby, cocaine administration would increase the likelihood of responding to a stimulus, while cannabis administration would 
decrease the likelihood of responding. The enhancement of the direct pathway by cocaine might increase long-term potentiation (LTP) and thereby facilitate learning of cue-drug associations (Frank, 2005). This might explain why addiction to cocaine is more prevalent than addiction to cannabis (Peer et al., 2013).

The reduction in thalamic activation during cocaine and cannabis intoxication as compared to placebo however may be primarily related to a drug induced deactivation of the hyperdirect pathway. Drugs of abuse have been shown to decrease the function of the thalamus in gating of stimuli (Ding et al., 2010; Dissanayake et al., 2013; Doherty et al., 2008; Green et al., 2003). Therefore, it is possible that under the influence of both drugs, the initial inhibition of the thalamus via the hyperdirect pathway was so large that subsequent activations or deactivations via the direct or indirect pathways were not large enough to be distinguished in the BOLD signal. The difference in conduction speed between the hyperdirect and indirect/direct pathway has been speculated to be as brief as $22 \mathrm{~ms}$ (Chambers, Garavan, \& Bellgrove, 2009).

\section{Mood and psychedelic effects of drugs of abuse}

Chapters 3 and 6 focused on the effect of cannabis, cocaine and MDMA on mood and psychedelic experiences. As expected, cocaine and cannabis intoxication had opposing effects on mood. Subjects under the influence of cocaine were generally activated and aroused, while cannabis intoxication showed the opposite effect. In chapter 6 we showed that the effect of MDMA on mood is ambiguous, increasing both positive (vigor, friendliness, elation and arousal) and negative (anxiousness, confusion) affect. Opposing results of cannabis, cocaine and MDMA on mood could be due to their differential effects on serotonin levels in the brain. MDMA and cocaine have been shown to increase levels of serotonin acutely, while cannabis administration has been shown to decrease serotonin function (Anastasio et al., 2011; Broderick, Olabisi, Rahni, \& Zhou, 2004; Darmani, 2001; Gudelsky \& Yamamoto, 2008; Nakazi, Bauer, Nickel, Kathmann, \& Schlicker, 2000; Nonkes, van Bussel, Verheij, \& Homberg, 2011). Although cocaine is generally known to increase dopamine and noradrenaline it also increases serotonin in the synaptic cleft by blocking the serotonin reuptake transporter (SERT). MDMA also increases serotonin, however, it exerts its effect through a different mechanism, namely reversal of the SERT, thereby causing direct release of serotonin (Dumont \& Verkes, 2006; Nonkes et al., 2011). Cannabis influences serotonin 
more indirectly. Agonism at $\mathrm{CB}_{1}$ receptors has been shown to decrease serotonin levels through a pre-synaptic feedback mechanism in the mouse brain (Nakazi et al., 2000). Low levels of serotonin have been linked to the occurrence of depression, and drugs increasing serotonin levels are the first pharmacological treatment option for depression (Hensler, 2006). However, to date these drugs have only limited efficacy, with only $30-40 \%$ of patients achieving full remission (Artigas, 2013). Antidepressant drugs that lead to sustained remission in the majority of depressed patients do not exist yet. Finding more efficient alternative drug targets is therefore of great importance. One option is through the addition of $5-\mathrm{HT}_{2 \mathrm{~A}}$ agonists to antidepressant treatment. This is discussed more in-depth in the section on 5-HT receptor antagonism below.

The study described in chapter $\mathbf{3}$ is among the first to measure the effects of cocaine on dissociation and psychedelic experiences. Acute administration of cannabis and cocaine was shown to increase the amount of dissociation and psychedelic feelings experienced by the participants. The results are in accordance with studies investigating the effects of other stimulants such as amphetamines on these measures (Curran et al., 2004). The psychedelic and dissociative effects of cannabis in this study confirmed previous reports (Ballard et al., 2012; D'Souza et al., 2008, 2012; Stokes et al., 2009). The results are especially interesting in light of epidemiological studies associating extensive cannabis or cocaine use with psychotic disorders (Bossong \& Niesink, 2010; Morton, 1999; Roncero et al., 2012). Alterations in the modulatory role of dopamine in optimizing the signal-to-noise ratio in cortical circuits could explain the occurrence of the psychotic-like symptoms seen in our study. It has previously been shown that decreases in the ratio of $D_{1} / D_{2}$ receptors influences the attribution of internal and external stimuli, a key feature of schizophrenia (Winterer \& Weinberger, 2004). Preclinical studies have shown that acute administration of THC and cocaine increases $D_{2}$ availability in the striatum, whereas chronic THC administration lowers levels of $D_{1}$ receptors, resulting in an abnormal $D_{1} / D_{2}$ ratio (Briand, Flagel, Seeman, \& Robinson, 2008; Ginovart et al., 2012). Therefore, both the psychotomimetic symptoms and psychotic symptoms might share a common mechanism i.e. the abnormal ratio of $D_{1} / D_{2}$ receptors. Although none of the subjects in the study experienced a psychosis, the results indicate that even in healthy drug-users psychotic-like symptoms can occur. Studying the mechanisms behind the occurrence of psychotic-like symptoms after drug use in healthy subjects could provide insight in the etiology of schizophrenic disorders. 


\section{Trait impulsivity modulates drug induced mood changes}

While chapters 2 and $\mathbf{4}$ focus mainly on the acute effects of cannabis and cocaine on impulsivity and other neuropsychological measures, in chapter $\mathbf{3}$ the interaction between trait impulsivity and mood, dissociative states and psychedelic experiences was investigated. It was found that subjects with high trait impulsivity levels were shown to have an increased sensitivity to the psychedelic and dissociative effects produced by cannabis and cocaine respectively. High trait impulsivity can be linked to a number of impulse control disorders, such as Attention Deficit/Hyperactivity Disorder (ADHD), Parkinson's Disease and addiction (Aarts, van Holstein, \& Cools, 2011; de Wit, 2008; Volkow, Fowler, Wang, Baler, \& Telang, 2009). In addition, it has been shown that a relationship between a high level of trait impulsivity and an increased risk of developing and maintaining addiction exists (Belin et al., 2008; Economidou et al., 2009; Pattij \& De Vries, 2013). Why persons with high impulsivity levels are more sensitive to develop an addiction than others remains one of the most challenging questions in addiction research. Jupp \& Dalley (2013) report a number of studies that show that high trait impulsivity can be linked to a decrease in $D_{2}$ receptors in the brain. Their review supports the idea that abnormal endogenous dopamine transmission might be the basis for propensity for addiction. Dopamine released from the presynaptic neuron binds to postsynaptic $D_{2}$ receptors. A decrease in the number of $D_{2}$ receptors leads to increased availability of dopamine. This in turn may heighten impulsivity levels, sensitivity to drug cues and motivation to take drugs. Moreover, sustained drug use has also been shown to decrease levels of $D_{2}$, putting predisposed drug users at an even high risk of drug seeking behavior (Volkow et al., 2009). Seemingly incongruous with this theory is the recent finding of Caprioli et al. (2013) that high-impulsive rats with decreased $D_{2}$ availability showed an increase in these receptors after chronic cocaine use. However, since this finding seems to be specific for high impulsive rats, it might be the case that they were self-medicating their initial impulsiveness. Thus, this suggests that in order to prevent addiction, persons should be screened for decreased $D_{2}$ receptor functioning since a decrease in these receptors has been associated with increased risk of addiction. Consequently, high impulsive persons with decreased $D_{2}$ availability might improve control over their impulses and thereby decrease the risk of drug abuse when treated with dopamine agonists. 
Are MDMA induced memory deficits and alterations in impulsivity and mood prevented by pretreatment with a $5-\mathrm{HT}_{1}$ or a $5-\mathrm{HT}_{2}$ antagonist?

From our results described in chapter 5 and 6, it appears that neither the $5-\mathrm{HT}_{1}$ nor the 5$\mathrm{HT}_{2}$ antagonist interacted with the MDMA-induced impairments on tasks measuring cognitive or motor impulsivity, possibly because the magnitude of the effects were too small or because the tasks used were not sensitive enough to measure such effects. Chapter $\mathbf{5}$ and 6 suggest that the $5-\mathrm{HT}_{2}$ receptor is selectively involved in altering positive mood and verbal memory impairment.

Our results form an extension on earlier research showing that $5-\mathrm{HT}_{2 \mathrm{~A}}$ receptor agonism improves mood (Kometer et al., 2012; Landolt \& Wehrle, 2009; Matthias E. Liechti \& Vollenweider, 2001), but disprove theories that $5-\mathrm{HT}_{1}$ agonism does the same (Artigas, 2013). However, our findings on the absence of involvement of the $5-\mathrm{HT}_{1}$ receptor should be interpreted with caution, since the $5-\mathrm{HT}_{1}$ antagonist used (pindolol) only partially blocks the $5-\mathrm{HT}_{1}$ receptor.

As discussed previously, the need for an efficacious antidepressant is high. It has been suggested that adding $5-\mathrm{HT}_{2}$ blockers to treatment for MDD might improve the therapeutic efficacy of selective serotonin reuptake inhibitors (SSRIs) (Artigas, 2013). Our results argue against the viability of such an approach. Our study indicates that the addition of $5-\mathrm{HT}_{2 \mathrm{~A}}$ agonists to SSRI therapy could be beneficial. Likewise, animal research has already shown that $5-\mathrm{HT}_{2 \mathrm{~A}}$ agonism potentiates the effects of venlafaxine and paroxetine (Nic Dhonnchadha, Ripoll, Clénet, Hascoët, \& Bourin, 2005). However, comparable studies in humans have not been done. Our data indicate that agonism at $5-\mathrm{HT}_{2 \mathrm{~A}}$ receptors might at least partially explain the previously found antidepressant effects of MDMA or even of hallucinogenic drugs such as ketamine (Aan Het Rot, Zarate, Charney, \& Mathew, 2012). In this recent review, Aan Het Rot, Zarate, Charney, \& Mathew (2012) illustrate the efficacy of ketamine as an antidepressant in patients suffering from treatment-resistant major depressive disorder (MDD). Although the main neurobiological mechanism of the antidepressant action of ketamine has been proposed to be through modulation of glutamatergic NMDA receptors, research by Kapur and Seeman (2002) shows that the binding potential of ketamine to the $5-\mathrm{HT}_{2 \mathrm{~A}}$ receptor is just as large. This suggests that coadministration of a $5-\mathrm{HT}_{2 \mathrm{~A}}$ agonist with conventional antidepressant treatment might help 
increase response to antidepressants. This would greatly decrease the burden of disease associated with major depressive disorder.

A further benefit of stimulation of $5-\mathrm{HT}_{2 \mathrm{~A}}$ receptors could be that this has been shown to facilitate consolidation and extinction of fear memory (Bombardi \& Di Giovanni, 2013). Next to the increase in feelings of social closeness and reducing of fear responses associated with MDMA administration, the effect of MDMA on emotional learning and fear extinction has been proposed to be essential in its efficacy in treatment of anxiety disorders (Johansen \& Krebs, 2009). During MDMA-assisted psychotherapy, the combination of these three properties provides a balance between activating the emotional engagement needed to deal with traumatic experiences and feeling safe while doing so. This therapy-enhancing property of MDMA has been utilized in two recent trials. Treatment-resistant post-traumatic stress disorder (PTSD) patients who were treated with MDMA in addition to psychotherapy, experienced significantly less anxious symptoms at follow-up (Mithoefer et al., 2013; Oehen, Traber, Widmer, \& Schnyder, 2013). Thus, the finding that MDMA-induced mood changes are mediated trough activation of the $5-\mathrm{HT}_{2 \mathrm{~A}}$ receptor could improve treatment of severely debilitating disorders such as PTSD and MDD.

In chapter 5 it was shown that acute administration of MDMA decreased memory function, an effect that has been well-documented previously (Kuypers, de la Torre, Farre, Pujadas, \& Ramaekers, 2013; Kuypers \& Ramaekers, 2005; Kuypers, Wingen, Heinecke, Formisano, \& Ramaekers, 2011; Ramaekers \& Kuypers, 2006). Memory deficits after MDMA administration have been shown to be transient and suggested not to be directly associated with the amount of serotonin available in the synaptic cleft (Kuypers \& Ramaekers, 2005, 2007; Reneman et al., 2001). On the other hand, direct and indirect activation of the $5-\mathrm{HT}_{1}$ and $5-\mathrm{HT}_{2}$ receptors has been shown to be involved in mediating memory and learning processes (Galizio et al., 2009; Kay et al., 2010; Meneses, 1999; Trigo et al., 2008; Yasuno et al., 2003). However, so far it was unclear through which exact neuropharmacological mechanism MDMA exerted its effects on memory. The study in chapter 5 showed that pretreatment with $5-\mathrm{HT}_{2}$ blocker ketanserin prevented this MDMA-induced memory impairment on the Word Learning task, but had no effect on the other memory tasks. Stimulation of the $5-H T_{2}$ receptor by MDMA has been shown to increase stimulation of $D_{1} / D_{5}$ receptors, which in turn affects NMDA activation. By this process, the occurrence of LTP is increased, which can lead to saturation of the hippocampus and consequently, impairments 
in learning (Rozas et al., 2012). This would also explain why in our study, we only saw an effect of the $5-\mathrm{HT}_{2}$ antagonist on MDMA-induced memory failure in the WLT, since that is the only task requiring explicit learning. However, while increases in LTP in healthy subjects can have a negative effect, it seems that it might be advantageous for the treatment of depression. In animal models of depression, it has been shown that animals exposed to longterm stress to mimic MDD, LTP is decreased (Ryan, Anwyl, \& Rowan, 2008). Therefore it is no surprise that administration of a $5-\mathrm{HT}_{2}$ agonist has been shown to negate the decrease in LTP associated with depressive symptoms (Rozas et al., 2012; Ryan et al., 2008). In this way, the increase in LTP induced by $5-\mathrm{HT}_{2}$ receptor agonism could be beneficial in treating MDD. Until recently, it was not possible to acutely investigate the effect of 5- $\mathrm{HT}_{2}$ agonism on LTP in humans. However, the methodology discussed by Clapp, Hamm, Kirk and Teyler (2012) to measure LTP using non-invasive techniques like EEG or fMRI provides a new avenue of research on the effects of $5-\mathrm{HT}_{2}$ agonists on LTP in healthy and depressed humans.

The $5-\mathrm{HT}_{1}$ blocker pindolol did not affect memory impairment after MDMA administration. The lack of effect following $5-\mathrm{HT}_{1}$ receptor antagonism confirms recent studies which question the role of this receptor in memory (Hasler et al., 2009). Instead, the abundance of $5-\mathrm{HT}_{1}$ receptors in the hippocampus could be explained by its role in neurogenesis. Stimulation of the $5-\mathrm{HT}_{1}$ receptor has been shown to increase adult neurogenesis which is hypothesized to underlie the delayed antidepressant effect of SSRIs (Lacivita et al., 2012; Santarelli et al., 2003).

In sum, our results suggest that the $5-\mathrm{HT}_{2}$, but not the $5-\mathrm{HT}_{1}$, receptor is selectively involved in both the verbal memory impairment and positive mood changes seen after MDMA administration. The involvement of the $5-\mathrm{HT}_{2}$ receptor in both of these processes suggests a role for $5-\mathrm{HT}_{2}$ agonists in the treatment of MDD and PTSD. However, more research should be done on the efficacy and tolerability of such a therapy.

\section{Future research}

There are a number of new research avenues that could be investigated following the results in this thesis. Firstly, it was suggested that the differential response patterns to cannabis and cocaine administration during impulsivity tasks were due to the drugs influencing different impulse control systems in the brain. This might also be connected to the finding that among addicts, one can distinguish those that are less sensitive to errors and those that are less 
sensitive to reward. Consequently, different types of addicts should be treated in a different way. Clinical studies examining the effects of GABA agonists or glutamatergic antagonists on addiction should shed light on this mechanism.

Secondly, it was shown that thalamic deactivation was related to a decrease or an increase in reward sensitization after respectively cannabis and cocaine administration. This indicates that cannabis and cocaine exert their effects on different thalamocortical pathways through their modulation of the dopamine level. It is known that alteration of DA systems in adolescence might predispose teens for problematic drug use by changing the way reward circuits function (Baler \& Volkow, 2012; Chambers, Taylor, \& Potenza, 2003; Volkow, Wang, Fowler, \& Tomasi, 2012). However, how this relates to the acute effects of cannabis and cocaine is up to now unknown. Therefore, future research should focus on employing prospective drug studies in young subjects at risk of using drugs to see how single and repeated cannabis and cocaine doses affect reward circuits. This way, a better understanding of the etiology of addiction and the neurobiological and cognitive consequences of drug use could be developed.

Furthermore, the studies described in this dissertation indicate that high impulsive persons might be more sensitive to the subjective effects of cannabis and cocaine and consequently might be more likely to develop drug use problems. It was hypothesized that this was due to abnormal $D_{2}$-receptor functioning, but it is unclear if it is the amount of $D_{2}$ receptors that is responsible for the increased sensitivity to drug use in high impulsive individuals. Recent research has indicated that the reason for this ambiguity might be that the $D_{2}$ receptor can exist in two states, the high and low affinity state. In the high affinity state, D2 receptors are hypersensitive to dopamine and dopaminergic drugs, which increases the likelihood of developing an addiction (Briand et al., 2008; Samaha, 2013). Further studies should focus on examining how the $D 2^{\text {high }}$ receptor is influenced by drug use and whether persons with high trait impulsivity show increases or decreases in this receptor. However, PET studies in humans regarding the D $2^{\text {high }}$ receptors are difficult, since as of yet, no selective PET tracer for this receptor state exists that has been approved for human use. Finally, it was concluded that the $5-\mathrm{HT}_{2 \mathrm{~A}}$ but not the $5-\mathrm{HT}_{1}$ receptor was selectively involved in memory and mood alterations following MDMA administration. Some caution should be applied here, since the substance used to block $5-\mathrm{HT}_{1}$ (pindolol) is possibly not selective enough to fully block the $5-\mathrm{HT}_{1}$ receptor since it blocks this receptor only for $40 \%$. Thus, the 
lack of effects following 5- $\mathrm{HT}_{1}$ blockade could be due to insufficient antagonism instead of a true absence of effect. However, up to now, no suitably selective $5-\mathrm{HT}_{1}$ blocker approved for human use has been developed. Future research should clarify whether these effects are specific to MDMA or if the positive and negative mood changes following cocaine and cannabis respectively can also be negated after administration of a $5-\mathrm{HT}_{2 \mathrm{~A}}$ antagonist. Furthermore, following development of a full 5- $\mathrm{HT}_{1}$ blocker, it should be clarified whether mood and memory modulation are truly limited to the $5-\mathrm{HT}_{2}$ receptor. If this is the case, treatment for mood disorders or PTSD could benefit from addition of a $5-\mathrm{HT}_{2}$ receptor agonist in order to increase medication efficacy.

\section{Conclusion}

The results in this dissertation show that cocaine and cannabis affect impulsive responding in opposing ways. It was hypothesized that cannabis intoxication deactivates responding in the reflective system, while under the influence of cocaine the impulsive system is hyperactive. Since cannabis and cocaine seem to work on opposing systems, addiction to these substances should be treated accordingly. Possible treatments for deficits in reflective impulsivity include administration of glutamate antagonists, while persons with deficits in impulsive responding could profit more from medication that increases GABA. However, this does not explain why some individuals get addicted while others do not. One possibility lies in differences between the response of high and normal impulsive individuals to drugs of abuse. Our research shows that subjects with high trait impulsivity showed increased subjective responding to cannabis and cocaine. It was hypothesized that this could be due to decreases in the number of $D_{2}$-receptors in highly impulsive individuals. The subsequent increase in dopamine might make highly impulsive persons more susceptible to develop problematic drug use. Another factor involved in the etiology of drug addiction is the alteration of brain reward mechanism by drugs of abuse. In an fMRI study, both cannabis and cocaine were shown to deactivate the thalamus relative to placebo during a reward anticipation task. The altered response of the thalamus to reward after drug administration might indicate dysfunction of reward pathways.

Administration of cannabis and cocaine significantly increased ratings of dissociation and psychedelic experiences. It was hypothesized that this might be due to the effects these drugs have on the ratio of $D_{1} / D_{2}$ receptors. Alterations in the ratio of these receptors have 
been implicated in the etiology of schizophrenia. The psychotomimetic symptoms seen after cannabis and cocaine administration and psychotic symptoms seen in schizophrenia might share a common mechanism. Therefore, models of drug-induced psychotomimetic symptoms may serve research on psychosis and psychopathologies such as schizophrenia.

Lastly, $5-\mathrm{HT}_{2}$ but not $5-\mathrm{HT}_{1}$ receptors were shown to be selectively involved in modulation of MDMA-induced memory deficits and positive mood. It was suggested that a $5-\mathrm{HT}_{2}$ receptor agonist might be a useful addition to treatment of MDD and PTSD.

In summary, the studies described in this dissertation provide new avenues for future addiction and mood disorder research. Additionally, they invite thinking about different approaches to treating addiction and possibilities to prevent or diminish the risk of developing drug use problems. 
$118 \mid$ 


\section{REFERENCES}


Aan Het Rot, M., Zarate, C. A, Charney, D. S., \& Mathew, S. J. (2012). Ketamine for depression: where do we go from here? Biological psychiatry, 72, 537-47. doi:10.1016/j.biopsych.2012.05.003

Aarts, E., van Holstein, M., \& Cools, R. (2011). Striatal Dopamine and the Interface between Motivation and Cognition. Frontiers in psychology, 2, 163. doi:10.3389/fpsyg.2011.00163

Ameri, A. (1999). The effects of cannabinoids on the brain. Progress in neurobiology, 58, 315-48.

Anastasio, N. C., Stoffel, E. C., Fox, R. G., Bubar, M. J., Rice, K. C., Moeller, F. G., \& Cunningham, K. A. (2011). Serotonin (5-hydroxytryptamine) 5-HT(2A) receptor: association with inherent and cocaine-evoked behavioral disinhibition in rats. Behavioural pharmacology, 22, 248-61. doi:10.1097/FBP.0b013e328345f90d

Artigas, F. (in press). Developments in the field of antidepressants, where do we go now? European Neuropsychopharmacology. doi:10.1016/j.euroneuro.2013.04.013

Baker, T. E., Stockwell, T., Barnes, G., \& Holroyd, C. B. (2011). Individual differences in substance dependence: at the intersection of brain, behaviour and cognition. Addiction biology, 16, 458-66. doi:10.1111/j.1369-1600.2010.00243.x

Baler, R. D., \& Volkow, N. D. (2012). Addiction as a systems failure: focus on adolescence and smoking. Journal of the American Academy of Child and Adolescent Psychiatry, 50, 329339. doi:10.1016/j.jaac.2010.12.008.Addiction

Ballard, M. E., Bedi, G., \& de Wit, H. (2012). Effects of delta-9-tetrahydrocannabinol on evaluation of emotional images. Journal of Psychopharmacology, 26, 1289-98. doi:10.1177/0269881112446530

Ballard, M. E., Gallo, D. A, \& de Wit, H. (2013). Pre-encoding administration of amphetamine or THC preferentially modulates emotional memory in humans. Psychopharmacology, 226, 515-29. doi:10.1007/s00213-012-2924-5

Barkus, E., Morrison, P. D., Vuletic, D., Dickson, J. C., Ell, P. J., Pilowsky, L. S., ... Murray, R. M. (2011). Does intravenous $\Delta 9$-tetrahydrocannabinol increase dopamine release? A SPET study. Journal of Psychopharmacology, 25, 1462-1468. doi:10.1177/0269881110382465

Battistella, G., Fornari, E., Thomas, A., Mall, J.-F., Chtioui, H., Appenzeller, M., ... Giroud, C. (2013). Weed or Wheel! fMRI, Behavioural, and Toxicological Investigations of How Cannabis Smoking Affects Skills Necessary for Driving. PLOS ONE, 8, e52545. doi:10.1371/journal.pone.0052545

Baunez, C., \& Lardeux, S. (2011). Frontal cortex-like functions of the subthalamic nucleus. Frontiers in systems neuroscience, 5, 83. doi:10.3389/fnsys.2011.00083 
Bechara, A. (2005). Decision making, impulse control and loss of willpower to resist drugs: a neurocognitive perspective. Nature neuroscience, 8, 1458-63. doi:10.1038/nn1584

Bedi, G., Hyman, D., \& de Wit, H. (2010). Is ecstasy an "empathogen"? Effects of $\pm 3,4$ methylenedioxymethamphetamine on prosocial feelings and identification of emotional states in others. Biological psychiatry, 68, 1134-40. doi:10.1016/j.biopsych.2010.08.003

Belin, D., Mar, A. C., Dalley, J. W., Robbins, T. W., \& Everitt, B. J. (2008). High impulsivity predicts the switch to compulsive cocaine-taking. Science, 320, 1352-5. doi:10.1126/science.1158136

Benito, C., Tolón, R. M., Pazos, M. R., Núñez, E., Castillo, A. L., \& Romero, J. (2008). Cannabinoid CB2 receptors in human brain inflammation. British journal of pharmacology, 153, 277-85. doi:10.1038/sj.bjp.0707505

Bernschneider-Reif, S., Oxler, F., \& Freudenmann, R. W. (2006). The origin of MDMA ("ecstasy")--separating the facts from the myth. Die Pharmazie, 61, 966-72.

Bjork, J. M., Knutson, B., Fong, G. W., Caggiano, D. M., Bennett, S. M., \& Hommer, D. W. (2004). Incentive-elicited brain activation in adolescents: similarities and differences from young adults. Journal of Neuroscience, 24, 1793-802. doi:10.1523/JNEUROSCI.4862-03.2004

Bombardi, C., \& Di Giovanni, G. (2013). Functional anatomy of 5-HT2A receptors in the amygdala and hippocampal complex: relevance to memory functions. Experimental Brain Research, 230, 427-39. doi:10.1007/s00221-013-3512-6

Bonci, A., Bernardi, G., Grillner, P., \& Mercuri, N. B. (2003). The dopamine-containing neuron: maestro or simple musician in the orchestra of addiction? Trends in Pharmacological Sciences, 24, 172-177. doi:10.1016/S0165-6147(03)00068-3

Borg, J. (2008). Molecular imaging of the $5-\mathrm{HT}(1 \mathrm{~A})$ receptor in relation to human cognition. Behavioural brain research, 195, 103-11. doi:10.1016/j.bbr.2008.06.011

Borg, J., Andrée, B., Lundberg, J., Halldin, C., \& Farde, L. (2006). Search for correlations between serotonin 5-HT1A receptor expression and cognitive functions--a strategy in translational psychopharmacology. Psychopharmacology, 185, 389-94. doi:10.1007/s00213-006-0329-z

Bosker, W. M., Kuypers, K. P. C., Conen, S., \& Ramaekers, J. G. (2010). Dose-related effects of MDMA on psychomotor function and mood before, during, and after a night of sleep loss. Psychopharmacology, 209, 69-76. doi:10.1007/s00213-009-1767-1

Bossong, M. G., \& Niesink, R. J. M. (2010). Adolescent brain maturation, the endogenous cannabinoid system and the neurobiology of cannabis-induced schizophrenia. Progress in neurobiology, 92, 370-85. doi:10.1016/j.pneurobio.2010.06.010 
Bossong, M. G., van Berckel, B. N. M., Boellaard, R., Zuurman, L., Schuit, R. C., Windhorst, A. D., ... Kahn, R. S. (2009). Delta 9-tetrahydrocannabinol induces dopamine release in the human striatum. Neuropsychopharmacology 34, 759-66. doi:10.1038/npp.2008.138

Bowdle, T., Radant, A., Cowley, D., Kharasch, E., Strassman, R., \& Roy-Byrne, P. (1998). Psychedelic effects of ketamine in healthy volunteers. Anesthesiology, 8, 82-88.

Breiter, H. C., Gollub, R. L., Weisskoff, R. M., Kennedy, D. N., Makris, N., Berke, J. D., ... Hyman, S. E. (1997). Acute effects of cocaine on human brain activity and emotion. Neuron, 19, 591-611.

Bremner, J. D., Krystal, J. H., Putnam, F. W., Southwick, S. M., Marmar, C., Charney, D. S., \& Mazure, C. M. (1998). Measurement of dissociative states with the ClinicianAdministered Dissociative States Scale (CADSS). Journal of traumatic stress, 11, 125-36. doi:10.1023/A:1024465317902

Briand, L. A., Flagel, S. B., Seeman, P., \& Robinson, T. E. (2008). Cocaine self-administration produces a persistent increase in dopamine D2high receptors. European neuropsychopharmacology, 18, 551-556. doi:10.1016/j.euroneuro.2008.01.002.Cocaine

Broderick, P. A, Olabisi, O. A, Rahni, D. N., \& Zhou, Y. (2004). Cocaine acts on accumbens monoamines and locomotor behavior via a $5-\mathrm{HT} 2 \mathrm{~A} / 2 \mathrm{C}$ receptor mechanism as shown by ketanserin: 24-h follow-up studies. Progress in neuro-psychopharmacology \& biological psychiatry, 28, 547-57. doi:10.1016/j.pnpbp.2004.01.007

Brogden, R., \& Sorkin, E. (1990). Ketanserin. A review of its pharmacodynamic and pharmacokinetic properties, and therapeutic potential in hypertension and peripheral vascular disease. Drugs, 40, 903-949.

Buckholtz, J. W., Treadway, M. T., Cowan, R. L., Woodward, N. D., Benning, S. D., Li, R., ... Zald, D. H. (2010). Mesolimbic dopamine reward system hypersensitivity in individuals with psychopathic traits. Nature neuroscience, 13, 419-21. doi:10.1038/nn.2510

Burns, M. (1991, February). Pharmacology of cocaine and its relationship to behavior impairment. In The effects of drugs on human performance and behavior: drugs and driving/drugs in the workplace. Symposium conducted at the American Academy of Forensic Sciences.

Burns, M. (1992, September). Cocaine effects on performance. Presentation conducted at the $12^{\text {th }}$ International conference on alcohol, drugs and traffic safety, Cologne, Germany.

Butler, G. K. L., \& Montgomery, A. M. J. (2004). Impulsivity, risk taking and recreational "ecstasy" (MDMA) use. Drug and alcohol dependence, 76, 55-62. doi:10.1016/j.drugalcdep.2004.04.003 
Cairns, E., \& Cammock, T. (1978). Development of a more reliable version of the Matching Familiar Figures Test. Developmental Psychology, 14, 555-560.

Canales, J. J., \& Iversen, S. D. (2000). Psychomotor-activating effects mediated by dopamine $\mathrm{D}(2)$ and $\mathrm{D}(3)$ receptors in the nucleus accumbens. Pharmacology, biochemistry, and behavior, 67, 161-8.

Caprioli, D., Hong, Y. T., Sawiak, S. J., Ferrari, V., Williamson, D. J., Jupp, B., ... Dalley, J. W. (2013). Baseline-dependent effects of cocaine pre-exposure on impulsivity and D2/3 receptor availability in the rat striatum: possible relevance to the attention-deficit hyperactivity syndrome. Neuropsychopharmacology 38, 1460-71. doi:10.1038/npp.2013.44

Chambers, C. D., Garavan, H., \& Bellgrove, M. a. (2009). Insights into the neural basis of response inhibition from cognitive and clinical neuroscience. Neuroscience and biobehavioral reviews, 33, 631-46. doi:10.1016/j.neubiorev.2008.08.016

Chambers, R. A., Taylor, J. R., \& Potenza, M. N. (2003). Developmental Neurocircuitry of Motivation in Adolescence: A Critical Period of Addiction Vulnerability. American Journal of Psychiatry, 160, 1041-1052.

Childers, S. R., \& Breivogel, C. S. (1998). Cannabis and endogenous cannabinoid systems. Drug and alcohol dependence, 51, 173-87.

Clapp, W. C., Hamm, J. P., Kirk, I. J., \& Teyler, T. J. (2012). Translating long-term potentiation from animals to humans: a novel method for noninvasive assessment of cortical plasticity. Biological psychiatry, 71, 496-502. doi:10.1016/j.biopsych.2011.08.021

Cohen, M. X., \& Frank, M. J. (2009). Neurocomputational models of basal ganglia function in learning, memory and choice. Behavioural brain research, 199, 141-56. doi:10.1016/j.bbr.2008.09.029

Cole, J. C., \& Sumnall, H. R. (2003). The pre-clinical behavioural pharmacology of 3,4methylenedioxymethamphetamine (MDMA). Neuroscience \& Biobehavioral Reviews, 27, 199-217. doi:10.1016/S0149-7634(03)00031-9

Congdon, E., \& Canli, T. (2008). A neurogenetic approach to impulsivity. Journal of personality, 76, 1447-84. doi:10.1111/j.1467-6494.2008.00528.x

Cools, R., Roberts, A. C., \& Robbins, T. W. (2008). Serotoninergic regulation of emotional and behavioural control processes. Trends in cognitive sciences, 12, 31-40. doi:10.1016/j.tics.2007.10.011

Crean, R. D., Crane, N. A., \& Mason, B. J. (2012). An evidence based review of acute and longterm effects of cannabis use on executive cognitive functions. Journal of Addiction Medicine, 5, 1-8. doi:10.1097/ADM.0b013e31820c23fa.An 
Curran, C., Byrappa, N., \& McBride, A. (2004). Stimulant psychosis: systematic review. The British journal of Psychiatry, 185, 196-204. doi:10.1192/bjp.185.3.196

Curran, H. V., \& Travill, R. A. (1997). Mood and cognitive effects of $\pm 3,4$ methylenedioxymethamphetamine (MDMA, "ecstasy"): week-end "high" followed by mid-week low. Addiction, 92, 821-831.

D’Souza, D. C., Fridberg, D. J., Skosnik, P. D., Williams, A., Roach, B., Singh, N., ... Mathalon, D. (2012). Dose-related modulation of event-related potentials to novel and target stimuli by intravenous $\Delta^{9}$-THC in humans. Neuropsychopharmacology 37, 1632-46. doi:10.1038/npp.2012.8

D’Souza, D. C., Ranganathan, M., Braley, G., Gueorguieva, R., Zimolo, Z., Cooper, T., ... Krystal, J. (2008). Blunted psychotomimetic and amnestic effects of delta-9tetrahydrocannabinol in frequent users of cannabis. Neuropsychopharmacology 33, 2505-16. doi:10.1038/sj.npp.1301643

Dalley, J. W., Everitt, B. J., \& Robbins, T. W. (2011). Impulsivity, compulsivity, and top-down cognitive control. Neuron, 69, 680-94. doi:10.1016/j.neuron.2011.01.020

Darmani, N. A. (2001). Cannabinoids of diverse structure inhibit two DOl-induced 5-HT(2A) receptor-mediated behaviors in mice. Pharmacology, biochemistry, and behavior, 68, 311-7.

Davidson, E. S., Finch, J. F., \& Schenk, S. (1993). Variability in subjective responses to cocaine: initial experiences of college students. Addictive behaviors, 18, 445-53.

De Bellis, M. D., Wang, L., Bergman, S. R., Yaxley, R. H., Hooper, S. R., \& Huettel, S. A. (2013). Neural mechanisms of risky decision-making and reward response in adolescent onset cannabis use disorder. Drug and alcohol dependence, 133, 134-45. doi:10.1016/j.drugalcdep.2013.05.020

De la Torre, R., Farré, M., Roset, P. N., Pizarro, N., Abanades, S., Segura, M., ... Camí, J. (2004). Human pharmacology of MDMA: pharmacokinetics, metabolism, and disposition. Therapeutic drug monitoring, 26, 137-44.

De Wit, H. (2008). Impulsivity as a determinant and a consequence of drug use: a review of underlying processes. Addiction biology, 14, 22-31.

De Wit, Harriet, Enggasser, J. L., \& Richards, J. B. (2002). Acute administration of damphetamine decreases impulsivity in healthy volunteers. Neuropsychopharmacology 27, 813-25. doi:10.1016/S0893-133X(02)00343-3

Deelman, D., Liebrand, W., Koning-Haanstra, M., \& van den Burg, W. (1980). Measurements of aphasic disorders. A brief description of the SAN-battery. Gerontologie, 11, 17-21.

Di Chiara, G., \& Imperato, A. (1988). Drugs abused by humans preferentially increase synaptic dopamine concentrations in the mesolimbic system of freely moving rats. 
Proceedings of the National Academy of Sciences of the United States of America, 85(14), 5274-8.

Ding, J. B., Guzman, J. N., Peterson, J. D., Goldberg, J. A, \& Surmeier, D. J. (2010). Thalamic Gating of Corticostriatal Signaling by Cholinergic Interneurons. Neuron, 67, 294-307. doi:10.1016/j.neuron.2010.06.017

Dissanayake, D. W. N., Mason, R., \& Marsden, C. A. (2013). Sensory gating, Cannabinoids and Schizophrenia. Neuropharmacology, 67, 66-77. doi:10.1016/j.neuropharm.2012.10.011

Doherty, J. M., Masten, V. L., Powell, S. B., Ralph, R. J., Klamer, D., Low, M. J., \& Geyer, M. A. (2008). Contributions of dopamine D1, D2, and D3 receptor subtypes to the disruptive effects of cocaine on prepulse inhibition in mice. Neuropsychopharmacology 33, 264856. doi:10.1038/sj.npp.1301657

Dumont, G. J. H., \& Verkes, R. J. (2006). A review of acute effects of 3,4methylenedioxymethamphetamine in healthy volunteers. Journal of Psychopharmacology, 20, 176-87. doi:10.1177/0269881106063271

Economidou, D., Pelloux, Y., Robbins, T. W., Dalley, J. W., \& Everitt, B. J. (2009). High impulsivity predicts relapse to cocaine-seeking after punishment-induced abstinence. Biological psychiatry, 65, 851-6. doi:10.1016/j.biopsych.2008.12.008

Elliott, R., Zahn, R., Deakin, J. F. W., \& Anderson, I. M. (2011). Affective cognition and its disruption in mood disorders. Neuropsychopharmacology 36, 153-82. doi:10.1038/npp.2010.77

European Monitoring Centre for Drugs and Drug Addiction. (2009). Polydrug Use: patterns and responses. Luxembourg: European Union.

European Monitoring Centre for Drugs and Drug Addiction. (2011). Annual drug update: 2011 in review. Luxembourg: European Union

European Monitoring Centre for Drugs and Drug Addiction. (2012). Annual report 2012: the state of the drugs problem in Europe. Luxembourg: European Union.

European Monitoring Centre for Drugs and Drug Addiction. (2010). Cocaine: A European Union perspective in the global context. Luxembourg.

Evenden, J. L. (1999). Varieties of impulsivity. Psychopharmacology, 146, 348-61.

Everitt, B. J., Belin, D., Economidou, D., Pelloux, Y., Dalley, J. W., \& Robbins, T. W. (2008). Neural mechanisms underlying the vulnerability to develop compulsive drug-seeking habits and addiction. Philosophical transactions of the Royal Society of London. Series B, Biological sciences, 363, 3125-35. doi:10.1098/rstb.2008.0089 
Fattinger, K., Benowitz, N. L., Jones, R. T., \& Verotta, D. (2000). Nasal mucosal versus gastrointestinal absorption of nasally administered cocaine. European journal of clinical pharmacology, 56, 305-10.

Faure, A., Richard, J. M., \& Berridge, K. C. (2010). Desire and dread from the nucleus accumbens: cortical glutamate and subcortical GABA differentially generate motivation and hedonic impact in the rat. PloS one, 5, e11223. doi:10.1371/journal.pone.0011223

Fillmore, M. T., \& Rush, C. R. (2006). Polydrug abusers display impaired discriminationreversal learning in a model of behavioural control. Journal of Psychopharmacology, 20, 24-32. doi:10.1177/0269881105057000

Fillmore, M. T., Rush, C. R., \& Hays, L. (2002). Acute effects of oral cocaine on inhibitory control of behavior in humans. Drug and alcohol dependence, 67, 157-67.

Fillmore, M. T., Rush, C. R., \& Hays, L. (2005). Cocaine improves inhibitory control in a human model of response conflict. Experimental Clinical Psychopharmacology, 13, 327-336.

Fillmore, M. T., Rush, C. R., \& Hays, L. (2006). Acute effects of cocaine in two models of inhibitory control: implications of non-linear dose effects. Addiction, 101, 1323-32. doi:10.1111/j.1360-0443.2006.01522.x

Fitzgerald, P. J. (2011). A neurochemical yin and yang: does serotonin activate and norepinephrine deactivate the prefrontal cortex? Psychopharmacology, 213, 171-82. doi:10.1007/s00213-010-1856-1

Flory, J. D., Manuck, S. B., Matthews, K. A, \& Muldoon, M. F. (2004). Serotonergic function in the central nervous system is associated with daily ratings of positive mood. Psychiatry research, 129, 11-9. doi:10.1016/j.psychres.2004.06.010

Foltin, R. W., \& Haney, M. (2004). Intranasal cocaine in humans: acute tolerance, cardiovascular and subjective effects. Pharmacology, biochemistry, and behavior, 78, 93-101. doi:10.1016/j.pbb.2004.02.018

Frank, M. J. (2005). Dynamic dopamine modulation in the basal ganglia: a neurocomputational account of cognitive deficits in medicated and nonmedicated Parkinsonism. Journal of cognitive neuroscience, 17, 51-72. doi:10.1162/0898929052880093

Friedman, N. P., \& Miyake, A. (2004). The relations among inhibition and interference control functions: a latent-variable analysis. Journal of experimental psychology. General, 133, 101-35. doi:10.1037/0096-3445.133.1.101

Galizio, M., McKinney, P., Cerutti, D. T., \& Pitts, R. C. (2009). Effects of MDMA, methamphetamine and methylphenidate on repeated acquisition and performance in rats. Pharmacology, biochemistry, and behavior, 94, 305-11. doi:10.1016/j.pbb.2009.09.010 
Garavan, H., Kaufman, J. N., \& Hester, R. (2008). Acute effects of cocaine on the neurobiology of cognitive control. Philosophical transactions of the Royal Society of London. Series B, Biological sciences, 363, 3267-76. doi:10.1098/rstb.2008.0106

Ginovart, N., Tournier, B. B., Moulin-Sallanon, M., Steimer, T., Ibanez, V., \& Millet, P. (2012). Chronic $\Delta^{9}$-tetrahydrocannabinol exposure induces a sensitization of dopamine $D_{2} / 3$ receptors in the mesoaccumbens and nigrostriatal systems. Neuropsychopharmacology 37, 2355-67. doi:10.1038/npp.2012.91

Goldstein, R. Z., \& Volkow, N. D. (2002). Drug Addiction and Its Underlying Neurobiological Basis: Neuroimaging Evidence for the Involvement of the Frontal Cortex. American Journal of Psychiatry, 159, 1642-1652. doi:10.1176/appi.ajp.159.10.1642

Goldstein, R. Z, Alia-Klein, N., Tomasi, D., Zhang, L., Cottone, L. A., ... Volkow, N. D. (2007). Decreased prefrontal cortical sensitivity to monetary reward is associated with impaired motivation and self-control in cocaine addiction. American journal of psychiatry, 164, 43-51.

Gonzalez, R. (2007). Acute and non-acute effects of cannabis on brain functioning and neuropsychological performance. Neuropsychology review, 17, 347-61. doi:10.1007/s11065-007-9036-8

Gouzoulis-Mayfrank, E., \& Daumann, J. (2009). Neurotoxicity of drugs of abuse - the case of methylenedioxyamphetamines (MDMA, ecstasy), and stimulant amphetamines. Dialogues in Clinical Neuroscience, 11, 305-317.

Green, A. R., Mechan, A. O., Elliott, J. M., Shea, E. O., \& Colado, M. I. (2003). The Pharmacology and Clinical Pharmacology of 3,4- Methylenedioxymethamphetamine ( MDMA , "Ecstasy "). Pharmacological Reviews, 55, 463-508. doi:10.1124/pr.55.3.3.463

Green, B., Kavanagh, D., \& Young, R. (2003). Being stoned: a review of self-reported cannabis effects. Drug and alcohol review, 22, 453-60. doi:10.1080/09595230310001613976

Gudelsky, G. A, \& Yamamoto, B. K. (2008). Actions of 3,4-methylenedioxymethamphetamine (MDMA) on cerebral dopaminergic, serotonergic and cholinergic neurons. Pharmacology, biochemistry, and behavior, 90, 198-207. doi:10.1016/j.pbb.2007.10.003

Haber, S. N., \& Knutson, B. (2010). The reward circuit: linking primate anatomy and human imaging. Neuropsychopharmacology 35, 4-26. doi:10.1038/npp.2009.129

Hadamitzky, M., \& Koch, M. (2009). Effects of acute intra-cerebral administration of the 5$\mathrm{HT}(2 \mathrm{~A} / \mathrm{C})$ receptor ligands $\mathrm{DOI}$ and ketanserin on impulse control in rats. Behavioural brain research, 204, 88-92. doi:10.1016/j.bbr.2009.05.021

Haney, M., Hart, C., Collins, E. D., \& Foltin, R. W. (2005). Smoked cocaine discrimination in humans: effects of gabapentin. Drug and alcohol dependence, 80, 53-61. doi:10.1016/j.drugalcdep.2005.03.006 
Hart, C.L., van Gorp, W., Haney, M., Foltin, R. W., \& Fischman, M. W. (2001). Effects of acute smoked marijuana on complex cognitive performance. Neuropsychopharmacology, 25, 757-65. doi:10.1016/S0893-133X(01)00273-1

Hart, C.L., Ilan, A. B., Gevins, A., Gunderson, E. W., Role, K., Colley, J., \& Foltin, R. W. (2010). Neurophysiological and cognitive effects of smoked marijuana in frequent users. Pharmacology, biochemistry, and behavior, 96, 333-41. doi:10.1016/j.pbb.2010.06.003

Hasler, F., Studerus, E., Lindner, K., Ludewig, S., \& Vollenweider, F. X. (2009). Investigation of serotonin-1A receptor function in the human psychopharmacology of MDMA. Journal of Psychopharmacology, 23, 923-35. doi:10.1177/0269881108094650

Hazekamp, A., Ruhaak, R., Zuurman, L., van Gerven, J., \& Verpoorte, R. (2006). Evaluation of a Vaporizing Device ( Volcano 1 ) for the Pulmonary Administration of Tetrahydrocannabinol. Journal of Pharmaceutical Sciences, 95, 1308-1317. doi:10.1002/jps

Henquet, C., van Os, J., Kuepper, R., Delespaul, P., Smits, M., Campo, J. A, \& Myin-Germeys, I. (2010). Psychosis reactivity to cannabis use in daily life: an experience sampling study. The British journal of psychiatry: the journal of mental science, 196, 447-53. doi:10.1192/bjp.bp.109.072249

Hensler, J. G. (2006). Serotonergic modulation of the limbic system. Neuroscience and biobehavioral reviews, 30, 203-14. doi:10.1016/j.neubiorev.2005.06.007

Herin, D. V, Liu, S., Ullrich, T., Rice, K. C., \& Cunningham, K. A. (2005). Role of the serotonin 5HT2A receptor in the hyperlocomotive and hyperthermic effects of (+)-3,4methylenedioxymethamphetamine. Psychopharmacology, 178, 505-13. doi:10.1007/s00213-004-2030-4

Hermans, E. J., Bos, P. A, Ossewaarde, L., Ramsey, N. F., Fernández, G., \& van Honk, J. (2010). Effects of exogenous testosterone on the ventral striatal BOLD response during reward anticipation in healthy women. Neurolmage, 52, 277-83. doi:10.1016/j.neuroimage.2010.04.019

Higgins, S. T., Bickel, W. K., Hughes, J. R., Lynn, M., Capeless, M. A., \& Fenwick, J. W. (1990). Effects of intranasal cocaine on human learning, performance and physiology. Psychopharmacology, 102, 451-458.

Horn, N. R., Dolan, M., Elliott, R., Deakin, J. F. W., \& Woodruff, P. W. R. (2003). Response inhibition and impulsivity: an fMRI study. Neuropsychologia, 41, 1959-1966. doi:10.1016/S0028-3932(03)00077-0

Jansma, J. M., van Hell, H. H., Vanderschuren, L. J. M. J., Bossong, M. G., Jager, G., Kahn, R. S., \& Ramsey, N. F. (2013). THC reduces the anticipatory nucleus accumbens response to reward in subjects with a nicotine addiction. Translational psychiatry, 3, e234. doi:10.1038/tp.2013.6 
Jentsch, J. D., Verrico, C. D., Le, D., \& Roth, R. H. (1998). Repeated exposure to delta 9tetrahydrocannabinol reduces prefrontal cortical dopamine metabolism in the rat. Neuroscience letters, 246, 169-72.

Jex, H. R., McDonnell, J. D., \& Phatak, A. V. (1966). A “critical” tracking task for manual control research. IEEE, 7, 138-145.

Jia, Z., Worhunsky, P. D., Carroll, K. M., Rounsaville, B. J., Michael, C., Pearlson, G. D., \& Potenza, M. N. (2011). An initial study of neural responses to monetary incentives as related to treatment outcome in cocaine dependence. Biological psychiatry, 70, 553560. doi:10.1016/j.biopsych.2011.05.008.AN

Johansen, P. Ф., \& Krebs, T. S. (2009). How could MDMA (ecstasy) help anxiety disorders? A neurobiological rationale. Journal of Psychopharmacology, 23, 389-91. doi:10.1177/0269881109102787

Johnson, B., Overton, D., Wells, L., Kenny, P., Abramson, D., Dhother, S., ... Bordnick, P. (1998). Effects of acute intravenous cocaine on cardiovascular function, human learning, and performance in cocaine addicts. Psychiatry research, 77, 35-42.

Jupp, B., \& Dalley, J. W. (in press). Behavioral endophenotypes of drug addiction: Etiological insights from neuroimaging studies. Neuropharmacology. doi:10.1016/j.neuropharm.2013.05.041

Kagan, J. (1966). Reflection--impulsivity: the generality and dynamics of conceptual tempo. Journal of abnormal psychology, 71, 17-24.

Kalivas, P. W., \& Volkow, N. D. (2005). The Neural Basis of Addiction: A Pathology of Motivation and Choice. American Journal of Psychiatry, 162, 1403-1413.

Kapur, S., \& Seeman, P. (2002). NMDA receptor antagonists ketamine and PCP have direct effects on the dopamine $\mathrm{D}(2)$ and serotonin 5-HT(2)receptors-implications for models of schizophrenia. Molecular psychiatry, 7, 837-44. doi:10.1038/sj.mp.4001093

Kauert, G. F., Iwersen-Bergmann, S., \& Toennes, S. W. (2006). Assay of Delta9tetrahydrocannabinol (THC) in oral fluid-evaluation of the OraSure oral specimen collection device. Journal of analytical toxicology, 30, 274-277.

Kaufmann, R. M., Kraft, B., Frey, R., Winkler, D., Weiszenbichler, S., Bäcker, C., ... Kress, H. (2009). Acute Psychotropic Effects of Oral Cannabis Extract with a Defined Content of $\Delta$ 9 -Tetrahydrocannabinol ( THC) in Healthy Volunteers, 43, 24-32.

Kay, C., Harper, D. N., \& Hunt, M. (2010). Differential effects of MDMA and scopolamine on working versus reference memory in the radial arm maze task. Neurobiology of learning and memory, 93, 151-6. doi:10.1016/j.nlm.2009.09.005

Kirby, L. G., Zeeb, F. D., \& Winstanley, C. A. (2011). Contributions of serotonin in addiction vulnerability. Neuropharmacology, 61, 421-32. doi:10.1016/j.neuropharm.2011.03.022 
Kirk, J. M., Doty, P., \& De Wit, H. (1998). Effects of expectancies on subjective responses to oral delta9-tetrahydrocannabinol. Pharmacology, biochemistry, and behavior, 59, 28793.

Kirkpatrick, M. G., Johanson, C.-E., \& de Wit, H. (2013). Personality and the acute subjective effects of d-amphetamine in humans. Journal of Psychopharmacology, 27, 256-64. doi:10.1177/0269881112472564

Knutson, B, Fong, G. W., Adams, C. M., Varner, J. L., \& Hommer, D. (2001). Dissociation of reward anticipation and outcome with event-related fMRI. Neuroreport, 12, 3683-7.

Knutson, B., Westdorp, A., Kaiser, E., \& Hommer, D. (2000). FMRI visualization of brain activity during a monetary incentive delay task. Neurolmage, 12, 20-7. doi:10.1006/nimg.2000.0593

Knutson, B., Bjork, J. M., Fong, G. W., Hommer, D., Mattay, V. S., \& Weinberger, D. R. (2004). Amphetamine modulates human incentive processing. Neuron, 43, 261-9. doi:10.1016/j.neuron.2004.06.030

Knutson, B., \& Greer, S. M. (2008). Anticipatory affect: neural correlates and consequences for choice. Philosophical transactions of the Royal Society of London. Series B, Biological sciences, 363, 3771-86. doi:10.1098/rstb.2008.0155

Kometer, M., Schmidt, A., Bachmann, R., Studerus, E., Seifritz, E., \& Vollenweider, F. X. (2012). Psilocybin biases facial recognition, goal-directed behavior, and mood state toward positive relative to negative emotions through different serotonergic subreceptors. Biological psychiatry, 72, 898-906. doi:10.1016/j.biopsych.2012.04.005

Koob, G. F., Sanna, P. P., \& Bloom, F. E. (1998). Neuroscience of Addiction. Neuron, 21, 467476.

Kreek, M. J., Nielsen, D. A, Butelman, E. R., \& LaForge, K. S. (2005). Genetic influences on impulsivity, risk taking, stress responsivity and vulnerability to drug abuse and addiction. Nature neuroscience, 8, 1450-7. doi:10.1038/nn1583

Kuepper, R., Ceccarini, J., Lataster, J., van Os, J., van Kroonenburgh, M., van Gerven, J. M. a, ... Henquet, C. (2013). Delta-9-tetrahydrocannabinol-induced dopamine release as a function of psychosis risk: 18F-fallypride positron emission tomography study. PloS one, 8, e70378. doi:10.1371/journal.pone.0070378

Kufahl, P. R., Li, Z., Risinger, R. C., Rainey, C. J., Wu, G., Bloom, A. S., \& Li, S.-J. (2005). Neural responses to acute cocaine administration in the human brain detected by $\mathrm{FMRI}$. Neurolmage, 28, 904-14. doi:10.1016/j.neuroimage.2005.06.039

Kuypers, K. P. C., de la Torre, R., Farre, M., Pujadas, M., \& Ramaekers, J. G. (2013). Inhibition of MDMA-induced increase in cortisol does not prevent acute impairment of verbal memory. British journal of pharmacology, 168, 607-17. doi:10.1111/j.14765381.2012.02196.x 
Kuypers, K. P. C., Samyn, N., \& Ramaekers, J. G. (2006). MDMA and alcohol effects, combined and alone, on objective and subjective measures of actual driving performance and psychomotor function. Psychopharmacology, 187, 467-75. doi:10.1007/s00213-0060434-z

Kuypers, K. P. C., Wingen, M., Samyn, N., Limbert, N., \& Ramaekers, J. G. (2007). Acute effects of nocturnal doses of MDMA on measures of impulsivity and psychomotor performance throughout the night. Psychopharmacology, 192, 111-9. doi:10.1007/s00213-006-0679-6

Kuypers, K. P. C, \& Ramaekers, J. G. (2005). Transient memory impairment after acute dose of 75mg 3.4-Methylene-dioxymethamphetamine. Journal of Psychopharmacology, 19, 633-9. doi:10.1177/0269881105056670

Kuypers, K. P. C, \& Ramaekers, J. G. (2007). Acute dose of MDMA (75 mg) impairs spatial memory for location but leaves contextual processing of visuospatial information unaffected. Psychopharmacology, 189, 557-63. doi:10.1007/s00213-006-0321-7

Kuypers, K. P. C, Wingen, M., Heinecke, A., Formisano, E., \& Ramaekers, J. G. (2011). MDMA intoxication and verbal memory performance: a placebo-controlled pharmaco-MRI study. Journal of Psychopharmacology, 25, 1053-61. doi:10.1177/0269881111405361

Lacivita, E., Di Pilato, P., De Giorgio, P., Colabufo, N. a, Berardi, F., Perrone, R., \& Leopoldo, M. (2012). The therapeutic potential of 5-HT1A receptors: a patent review. Expert opinion on therapeutic patents, 22, 887-902. doi:10.1517/13543776.2012.703654

Landolt, H.-P., \& Wehrle, R. (2009). Antagonism of serotonergic 5-HT2A/2C receptors: mutual improvement of sleep, cognition and mood? The European journal of neuroscience, 29, 1795-809. doi:10.1111/j.1460-9568.2009.06718.x

Lex, B. W., Mendelson, J. H., Bavli, S., Harvey, K., \& Mello, N. K. (1984). Effects of acute marijuana smoking on pulse rate and mood states in women. Psychopharmacology, 84, 178-187.

Lidow, M., Goldman-Rakic, P., Gallager, D., \& Rakic, P. (1989). Quantitative autoradiographic mapping of serotonin 5-HT1 and 5-HT2 receptors and uptake sites in the neocortex of the rhesus monkey. The journal of comparative neurology, 280, 27-42.

Liechti, M. E., Saur, M. R., Gamma, a, Hell, D., \& Vollenweider, F. X. (2000). Psychological and physiological effects of MDMA ("Ecstasy") after pretreatment with the 5-HT(2) antagonist ketanserin in healthy humans. Neuropsychopharmacology 23, 396-404. doi:10.1016/S0893-133X(00)00126-3

Liechti, M. E., Baumann, C., Gamma, A., \& Vollenweider, F. X. (1998). Acute Psychological Effects of ( MDMA, "Ecstasy ") are Attenuated by the Serotonin Uptake Inhibitor Citalopram. Neuropsychopharmacology, 22, 513-21. 
Liechti, M. E., \& Vollenweider, F. X. (2001). Which neuroreceptors mediate the subjective effects of MDMA in humans? A summary of mechanistic studies. Human psychopharmacology, 16, 589-598. doi:10.1002/hup.348

Lile, J. A, Kelly, T. H., \& Hays, L. R. (2012). Separate and combined effects of the GABA(B) agonist baclofen and $\triangle 9-T H C$ in humans discriminating $\triangle 9-T H C$. Drug and alcohol dependence, 126, 216-23. doi:10.1016/j.drugalcdep.2012.05.023

Linden, D. E. J. (2007). The working memory networks of the human brain. The Neuroscientist : a review journal bringing neurobiology, neurology and psychiatry, 13, 257-67. doi:10.1177/1073858406298480

Lingford-Hughes, A., Watson, B., Kalk, N., \& Reid, A. (2010). Neuropharmacology of addiction and how it informs treatment. British medical bulletin, 96, 93-110. doi:10.1093/bmb/ldq032

Liu, X., Hairston, J., Schrier, M., \& Fan, J. (2011). Common and distinct networks underlying reward valence and processing stages: a meta-analysis of functional neuroimaging studies. Neuroscience and biobehavioral reviews, 35, 1219-36. doi:10.1016/j.neubiorev.2010.12.012

Logan, G. D., \& Cowan, W. B. (1984). On the ability to inhibit thought and action: A theory of an act of control. Psychological Review, 91, 295-327. doi:10.1037/0033-295X.91.3.295

Lukas, S. E., Sholar, M., Lundahl, L. H., Lamas, X., Kouri, E., Wines, J. D., ... Mendelson, J. H. (1996). Sex differences in plasma cocaine levels and subjective effects after acute cocaine administration in human volunteers. Psychopharmacology, 125, 346-54.

Maldjian, J.A ., Laurienti, P. J., \& Burdette, J. H. (2004). Precentral gyrus discrepancy in electronic versions of the Talairach atlas. Neurolmage, 21, 450-455. doi:10.1016/j.neuroimage.2003.09.032

Maldjian, J. A. , Laurienti, P. J., Kraft, R. a., \& Burdette, J. H. (2003). An automated method for neuroanatomic and cytoarchitectonic atlas-based interrogation of $\mathrm{FMRI}$ data sets. Neurolmage, 19, 1233-1239. doi:10.1016/S1053-8119(03)00169-1

McDonald, J., Schleifer, L., Richards, J. B., \& de Wit, H. (2003). Effects of THC on behavioral measures of impulsivity in humans. Neuropsychopharmacology 28, 1356-65. doi:10.1038/sj.npp.1300176

McFarland, K., Lapish, C. C., \& Kalivas, P. W. (2003). Prefrontal glutamate release into the core of the nucleus accumbens mediates cocaine-induced reinstatement of drugseeking behavior. Journal of Neuroscience, 23, 3531-7.

McLellan, A., Lewis, D., O’Brien, C. P., \& Kleber, H. (2000). Drug dependence as a chronic medical illness. Implications for treatment, insurance and outcomes evaluation. Journal of the American Medical Association, 284, 1689-1695. 
Melis, M., \& Pistis, P. (2007). Endocannabinoid signaling in midbrain dopamine neurons: more than physiology? Current neuropharmacology, 5, 268-77. doi:10.2174/157015907782793612

Meneses, A. (1999). 5-HT system and cognition. Neuroscience and biobehavioral reviews, 23, 1111-25.

Meneses, A. (1999). Are 5-HT(1B/1D) and 5- $\mathrm{HT}(2 \mathrm{~A} / 2 \mathrm{~B} / 2 \mathrm{C})$ receptors involved in learning and memory processes? IDrugs, 2, 796-801.

Meneses, A. (2002). Involvement of 5-HT2A/2B/2C receptors on memory formation : Simple Agonism , Antagonism , or Inverse Agonism ? Cellular and Molecular Neurobiology, 22, 675-688.

Ménétrey, A., Augsburger, M., Favrat, B., Pin, M. A, Rothuizen, L. E., Appenzeller, M., ... Giroud, C. (2005). Assessment of driving capability through the use of clinical and psychomotor tests in relation to blood cannabinoids levels following oral administration of $20 \mathrm{mg}$ dronabinol or of a cannabis decoction made with 20 or $60 \mathrm{mg}$ Delta9-THC. Journal of analytical toxicology, 29, 327-38.

Messer, S. B. (1976). Reflection-impulsivity: A review. Psychological Bulletin, 83, 1026-1052. doi:10.1037/0033-2909.83.6.1026

Messer, S. B., \& Brodzinsky, D. M. (1981). Three-Year Stability of Reflection-Impulsivity in Young Adolescents. Developmental Psychology, 17, 848-850.

Metrik, J., Kahler, C. W., Reynolds, B., McGeary, J. E., Monti, P. M., Haney, M., ... Rohsenow, D. J. (2012). Balanced placebo design with marijuana: pharmacological and expectancy effects on impulsivity and risk taking. Psychopharmacology, 223, 489-99. doi:10.1007/s00213-012-2740-y

Mithoefer, M. C., Wagner, M. T., Mithoefer, A. T., Jerome, L., Martin, S. F., Yazar-Klosinski, B., ... Doblin, R. (2013). Durability of improvement in post-traumatic stress disorder symptoms and absence of harmful effects or drug dependency after 3,4methylenedioxymethamphetamine-assisted psychotherapy: a prospective long-term follow-up study. Journal of Psychopharmacology, 27, 28-39. doi:10.1177/0269881112456611

Morgan, M J. (1998). Recreational use of "ecstasy" (MDMA) is associated with elevated impulsivity. Neuropsychopharmacology, 19, 252-64. doi:10.1016/S0893$133 \times(98) 00012-8$

Morgan, M. J. (2000). Ecstasy (MDMA): a review of its possible persistent psychological effects. Psychopharmacology, 152, 230-248. doi:10.1007/s002130000545

Morgan, M. J., Impallomeni, L. C., Pirona, A., \& Rogers, R. D. (2006). Elevated impulsivity and impaired decision-making in abstinent Ecstasy (MDMA) users compared to polydrug 
and drug-naïve controls. Neuropsychopharmacology 31, 1562-73. doi:10.1038/sj.npp.1300953

Morton, W. A. (1999). Cocaine and Psychiatric Symptoms. Primary care companion to the Journal of clinical psychiatry, 1, 109-113.

Müller, C. P., Carey, R. J., Huston, J. P., \& De Souza Silva, M. A. (2007). Serotonin and psychostimulant addiction: focus on 5-HT1A-receptors. Progress in neurobiology, 81, 133-78. doi:10.1016/j.pneurobio.2007.01.001

Murphy, P. N., Wareing, M., \& Fisk, J. (2006). Users' perceptions of the risks and effects of taking ecstasy (MDMA): a questionnaire study. Journal of Psychopharmacology, 20, 447-55. doi:10.1177/0269881106063270

Nakazi, M., Bauer, U., Nickel, T., Kathmann, M., \& Schlicker, E. (2000). Inhibition of serotonin release in the mouse brain via presynaptic cannabinoid CB1 receptors. NaunynSchmiedeberg's archives of pharmacology, 361, 19-24.

Nambu, A., Tokuno, H., \& Takada, M. (2002). Functional significance of the corticosubthalamo-pallidal "hyperdirect" pathway. Neuroscience research, 43, 111-7.

Nestler, E. J. (2005). Is there a common molecular pathway for addiction? Nature neuroscience, 8, 1445-9. doi:10.1038/nn1578

Netherlands Institute of Mental Health and Addiction. (2012). Nationale Drug Monitor Jaarbericht 2011. Utrecht.

Nic Dhonnchadha, B. A., Ripoll, N., Clénet, F., Hascoët, M., \& Bourin, M. (2005). Implication of 5-HT2 receptor subtypes in the mechanism of action of antidepressants in the four plates test. Psychopharmacology, 179, 418-29. doi:10.1007/s00213-004-2044-y

Nonkes, L. J. P., van Bussel, I. P. G., Verheij, M. M. M., \& Homberg, J. R. (2011). The interplay between brain 5-hydroxytryptamine levels and cocaine addiction. Behavioural pharmacology, 22, 723-38. doi:10.1097/FBP.0b013e32834d6260

Oehen, P., Traber, R., Widmer, V., \& Schnyder, U. (2013). A randomized, controlled pilot study of MDMA ( $\pm 3,4$-Methylenedioxymethamphetamine)-assisted psychotherapy for treatment of resistant, chronic Post-Traumatic Stress Disorder (PTSD). Journal of Psychopharmacology, 27, 40-52. doi:10.1177/0269881112464827

Oswald, L. M., Wong, D. F., Zhou, Y., Kumar, A., Brasic, J., Alexander, M., ... Wand, G. S. (2007). Impulsivity and chronic stress are associated with amphetamine-induced striatal dopamine release. Neurolmage, 36, 153-66. doi:10.1016/j.neuroimage.2007.01.055

Panlilio, L. V, Solinas, M., Matthews, S. a, \& Goldberg, S. R. (2007). Previous exposure to THC alters the reinforcing efficacy and anxiety-related effects of cocaine in rats. Neuropsychopharmacology 32, 646-57. doi:10.1038/sj.npp.1301109 
Parrott, A. C., \& Lasky, J. (1998). Ecstasy (MDMA) effects upon mood and cognition: before, during and after a Saturday night dance. Psychopharmacology, 139, 261-8.

Parrott, A. C., Lock, J., Conner, a C., Kissling, C., \& Thome, J. (2008). Dance clubbing on MDMA and during abstinence from Ecstasy/MDMA: prospective neuroendocrine and psychobiological changes. Neuropsychobiology, 57, 165-80. doi:10.1159/000147470

Parrott, A. C. (2001). Human psychopharmacology of Ecstasy (MDMA): a review of 15 years of empirical research. Human psychopharmacology, 16, 557-577. doi:10.1002/hup.351

Parrott, A. C. (2012). MDMA and 5-HT neurotoxicity: the empirical evidence for its adverse effects in humans - no need for translation. British journal of pharmacology, 166, 151820. doi:10.1111/j.1476-5381.2012.01941.x

Parrott, A. C., \& Stuart, M. (1997). Ecstasy (MDMA), Amphetamine, and LSD : Comparative Mood Profiles in Recreational Polydrug Users. Human Psychopharmacology, 12, 501504.

Parvaz, M. ., Maloney, T., Moeller, S. J., Woicik, P. A., Alia-Klein, N., Telang, F., ... Goldstein, R. Z. (2012). Sensitivity to monetary reward is most severely compromised in recently abstaining cocaine addicted individuals: A cross-sectional ERP study. Psychiatry Research, 203, 75-82. doi:10.1016/j.pscychresns.2012.01.001.Sensitivity

Pattij, T., \& De Vries, T. J. (2013). The role of impulsivity in relapse vulnerability. Current Opinion in Neurobiology, 23, 1-6. doi:10.1016/j.conb.2013.01.023

Pattij, T., \& Vanderschuren, L. J. M. J. (2008). The neuropharmacology of impulsive behaviour. Trends in pharmacological sciences, 29, 192-9. doi:10.1016/j.tips.2008.01.002

Peer, K., Rennert, L., Lynch, K. G., Farrer, L., Gelernter, J., \& Kranzler, H. R. (2013). Prevalence of DSM-IV and DSM-5 alcohol, cocaine, opioid, and cannabis use disorders in a largely substance dependent sample. Drug and alcohol dependence, 127, 215-9. doi:10.1016/j.drugalcdep.2012.07.009

Penetar, D. M., Looby, A. R., Su, Z., Lundahl, L. H., Mcneil, J. F., Lukas, S. E., \& Ero, M. (2006). Benztropine pretreatment does not affect responses to acute cocaine administration in human volunteers. Human psychopharmacology, 21, 549-559. doi:10.1002/hup

Perales, J. C., Verdejo-Garcia, A., Moya, M., Lozano, O., \& Perez-Garcia, M. (2009a). Bright and dark sides of impulsivity: performance of women with high and low trait impulsivity on neuropsychological tasks. Journal of clinical and experimental neuropsychology, 31, 927-44. doi:10.1080/13803390902758793

Perales, J. C., Verdejo-Garcia, A., Moya, M., Lozano, O., \& Perez-Garcia, M. (2009b). Bright and dark sides of impulsivity: performance of women with high and low trait impulsivity on neuropsychological tasks. Journal of clinical and experimental neuropsychology, 31, 927-44. doi:10.1080/13803390902758793 
Perkonigg, A., Lieb, R., Höfler, M., Schuster, P., Sonntag, H., \& Wittchen, H. U. (1999). Patterns of cannabis use, abuse and dependence over time: incidence, progression and stability in a sample of 1228 adolescents. Addiction, 94, 1663-78.

Pettinati, H. M., Brien, C. P. O., \& Dundon, W. D. (2013). Current status of co-occurring mood and substance use disorders: a new therapeutic target. The American journal of psychiatry, 170, 23-30. doi:10.1176/appi.ajp.2012.12010112

Piechatzek, M., Indlekofer, F., Daamen, M., Glasmacher, C., Lieb, R., Pfister, H., ... Schütz, C. G. (2009). Is moderate substance use associated with altered executive functioning in a population-based sample of young adults ? Human Psychopharmacology, 24, 650-665. doi:10.1002/hup

Pierce, R. C., \& Kumaresan, V. (2006). The mesolimbic dopamine system: the final common pathway for the reinforcing effect of drugs of abuse? Neuroscience and biobehavioral reviews, 30, 215-38. doi:10.1016/j.neubiorev.2005.04.016

Pierce, R. C., \& Vassoler, F. M. (2013). Deep brain stimulation for the treatment of addiction: basic and clinical studies and potential mechanisms of action. Psychopharmacology, 229, 487-91. doi:10.1007/s00213-013-3214-6

Pistis, M., Porcu, G., Melis, M., Diana, M., \& Gessa, G. L. (2001). Effects of cannabinoids on prefrontal neuronal responses to ventral tegmental area stimulation. The European journal of neuroscience, 14, 96-102.

Pistis, M., Ferraro, L., Pira, L., Flore, G., Tanganelli, S., Luigi, G., \& Devoto, P. (2002). D 9 Tetrahydrocannabinol decreases extracellular GABA and increases extracellular glutamate and dopamine levels in the rat prefrontal cortex : an in vivo microdialysis study. Brain Research, 948, 155-158.

Pistis, M., Perra, S., Pillolla, G., Melis, M., Muntoni, A. L., \& Gessa, G. L. (2004). Adolescent exposure to cannabinoids induces long-lasting changes in the response to drugs of abuse of rat midbrain dopamine neurons. Biological psychiatry, 56, 86-94. doi:10.1016/j.biopsych.2004.05.006

Quednow, B. B., Kühn, K.-U., Hoppe, C., Westheide, J., Maier, W., Daum, I., \& Wagner, M. (2007). Elevated impulsivity and impaired decision-making cognition in heavy users of MDMA ("Ecstasy"). Psychopharmacology, 189, 517-30. doi:10.1007/s00213-005-0256-4

Rabiner, E. A., Gunn, R. N., Wilkins, M. R., Sargent, P. A., Mocaer, E., Sedman, E., ... Grasby, P. M. (2000). Drug action at the 5-HT(1A) receptor in vivo: autoreceptor and postsynaptic receptor occupancy examined with PET and [carbonyl-(11)C]WAY-100635. Nuclear medicine and biology, 27, 509-13.

Ramaekers, J. G., Moeller, M. R., van Ruitenbeek, P., Theunissen, E. L., Schneider, E., \& Kauert, G. (2006). Cognition and motor control as a function of Delta9-THC concentration in serum and oral fluid: limits of impairment. Drug and alcohol dependence, 85, 114-22. doi:10.1016/j.drugalcdep.2006.03.015 
Ramaekers, J. G., Kauert, G., Theunissen, E. L., Schneider, E., \& Moeller, M. R. (2006). Highpotency marijuana impairs executive function and inhibitory motor control. Neuropsychopharmacology 31, 2296-303. doi:10.1038/sj.npp.1301068

Ramaekers, J. G., Kauert, G., Theunissen, E. L., Toennes, S. W., \& Moeller, M. R. (2009). Neurocognitive performance during acute THC intoxication in heavy and occasional cannabis users. Journal of Psychopharmacology, 23, 266-77. doi:10.1177/0269881108092393

Ramaekers, J. G., \& Kuypers, K. P. C. (2006). Acute effects of 3,4methylenedioxymethamphetamine (MDMA) on behavioral measures of impulsivity: alone and in combination with alcohol. Neuropsychopharmacology 31, 1048-55. doi:10.1038/sj.npp.1300894

Ramaekers, J. G., Kuypers, K. P. C., Wingen, M., Heinecke, A., \& Formisano, E. (2009). Involvement of inferior parietal lobules in prospective memory impairment during acute MDMA (ecstasy) intoxication: an event-related fMRI study. Neuropsychopharmacology 34, 1641-8. doi:10.1038/npp.2008.219

Ramaekers, J. G., Theunissen, E. L., de Brouwer, M., Toennes, S. W., Moeller, M. R., \& Kauert, G. (2011). Tolerance and cross-tolerance to neurocognitive effects of THC and alcohol in heavy cannabis users. Psychopharmacology, 214, 391-401. doi:10.1007/s00213-0102042-1

Reneman, L., Lavalaye, J., Schmand, B., de Wolff, F. A, van den Brink, W., den Heeten, G. J., \& Booij, J. (2001). Cortical serotonin transporter density and verbal memory in individuals who stopped using 3,4-methylenedioxymethamphetamine (MDMA or "ecstasy"): preliminary findings. Archives of general psychiatry, 58, 901-6.

Reneman, L., Endert, E., de Bruin, K., Lavalaye, J., Feenstra, M., de Wolff, F., \& Booij, J. (2002). The Acute and Chronic Effects of MDMA ("Ecstasy ") on Cortical 5-HT 2A Receptors in Rat and Human Brain. Neuropsychopharmacology, 26, 387-396.

Rey, A. (1958). The clinical examination in psychology / L'examen clinique en psychologie. Oxford, England: Presses Universitaires de France.

Robinson, E. S. J., Dalley, J. W., Theobald, D. E. H., Glennon, J. C., Pezze, M. a, Murphy, E. R., \& Robbins, T. W. (2008). Opposing roles for 5-HT2A and 5-HT2C receptors in the nucleus accumbens on inhibitory response control in the 5-choice serial reaction time task. Neuropsychopharmacology 33, 2398-406. doi:10.1038/sj.npp.1301636

Roncero, C., Ros-Cucurull, E., Daigre, C., \& Casas, M. (2012). Prevalence and risk factors of psychotic symptoms in cocaine-dependent patients. Actas españolas de psiquiatría, 4, 187-97.

Rozas, C., Loyola, S., Ugarte, G., Zeise, M. L., Reyes-Parada, M., Pancetti, F., ... Morales, B. (2012). Acutely applied MDMA enhances long-term potentiation in rat hippocampus 
involving D1/D5 and 5-HT2 receptors through a polysynaptic mechanism. European neuropsychopharmacology, 22, 584-95. doi:10.1016/j.euroneuro.2011.11.010

Rush, C. R., Baker, R. W., \& Wright, K. (1999). Acute physiological and behavioral effects of oral cocaine in humans: a dose-response analysis. Drug and alcohol dependence, 55(12), 1-12.

Ryan, B. K., Anwyl, R., \& Rowan, M. J. (2008). 5-HT2 receptor-mediated reversal of the inhibition of hippocampal long-term potentiation by acute inescapable stress. Neuropharmacology, 55(2), 175-82. doi:10.1016/j.neuropharm.2008.05.006

Sadzot, B., Baraban, J. M., Glennon, R. A, Lyon, R. A, Leonhardt, S., Jan, C. R., \& Titeler, M. (1989). Hallucinogenic drug interactions at human brain 5-HT2 receptors: implications for treating LSD-induced hallucinogenesis. Psychopharmacology, 98(4), 495-9.

Salkind, N., \& Wright, J. (1977). The development of reflection impulsivity and cognitive efficiency: An integrated model. Human Development, 20, 377-387.

Samaha, A.-N. (2013). Can antipsychotic treatment contribute to drug addiction in schizophrenia? Progress in neuro-psychopharmacology \& biological psychiatry. doi:10.1016/j.pnpbp.2013.06.008

Substance Abuse and Mental Health Services Administration. (2012). Results from the 2011 National Survey on Drug Use and Health : Summary of National Findings. Rockville, MD, United States of America.

Santarelli, L., Saxe, M., Gross, C., Surget, A., Battaglia, F., Dulawa, S., ... Hen, R. (2003). Requirement of hippocampal neurogenesis for the behavioral effects of antidepressants. Science, 301, 805-9. doi:10.1126/science.1083328

Schiff, N. D. (2008). Central thalamic contributions to arousal regulation and neurological disorders of consciousness. Annals of the New York Academy of Sciences, 1129, 105-18. doi:10.1196/annals.1417.029

Schmidt, J. (1987). Neurotoxicity of the Psychedelic Amphetamine, Methylenedioxymethamphetamine. The Journal of pharmacology and experimental therapeutics, 240, 1-7.

Schmitt, J. A. J., Kruizinga, M. J., \& Riedel, W. J. (2001). Non-serotonergic pharmacological profiles and associated cognitive effects of serotonin reuptake inhibitors. Journal of Psychopharmacology, 15, 173-179. doi:10.1177/026988110101500304

Schott, B. H., Seidenbecher, C. I., Richter, S., Wüstenberg, T., Debska-Vielhaber, G., Schubert, H., ... Düzel, E. (2011). Genetic variation of the serotonin 2a receptor affects hippocampal novelty processing in humans. PloS one, 6, e15984. doi:10.1371/journal.pone.0015984 
Schouw, M. L. J., De Ruiter, M. B., Kaag, a M., van den Brink, W., Lindauer, R. J. L., \& Reneman, L. (2013). Dopaminergic dysfunction in abstinent dexamphetamine users: results from a pharmacological $\mathrm{fMRI}$ study using a reward anticipation task and a methylphenidate challenge. Drug and alcohol dependence, 130, 52-60. doi:10.1016/j.drugalcdep.2012.10.010

Schreiber, R., \& de Vry, J. (1993). 5-HT1A Receptor ligands in animal models of anxiety, impulsivity and depression: multiple mechanisms of action? Progress in neuropsychopharmacology \& biological psychiatry, 17, 87-104.

Shallice, T. (1982). Specific impairments of planning. Philosophical transactions of the Royal Society of London. Series B, Biological Sciences, 298, 199-209.

Sharpley, A. L., Elliott, J. M., Attenburrow, M. J., \& Cowen, P. J. (1994). Slow wave sleep in humans: role of 5-HT2A and 5-HT2C receptors. Neuropharmacology, 33, 467-71.

Sigmund, J. C., Vogler, C., Huynh, K.-D., de Quervain, D. J.-F., \& Papassotiropoulos, A. (2008). Fine-mapping at the HTR2A locus reveals multiple episodic memory-related variants. Biological psychology, 79, 239-42. doi:10.1016/j.biopsycho.2008.06.002

Sokhadze, E. M., Baruth, J. M., Sears, L., Sokhadze, G. E., El-Baz, A. S., \& Casanova, M. F. (2012). Prefrontal neuromodulation using rTMS improves error monitoring and correction function in autism. Applied psychophysiology and biofeedback, 37, 91-102. doi:10.1007/s10484-012-9182-5

Solowij, N. (1998). Cannabis and cognitive functioning. Cambridge: Cambridge University Press.

Spronk, D. B., van Wel, J. H. P., Ramaekers, J. G., \& Verkes, R. J. (2013). Characterizing the cognitive effects of cocaine: A comprehensive review. Neuroscience and biobehavioral reviews, 37, 1838-1859. doi:10.1016/j.neubiorev.2013.07.003

Stanford, M. S., Mathias, C. W., Dougherty, D. M., Lake, S. L., Anderson, N. E., \& Patton, J. H. (2009). Fifty years of the Barratt Impulsiveness Scale: An update and review. Personality and Individual Differences, 47, 385-395. doi:10.1016/j.paid.2009.04.008

Stillman, R., Jones, R. T., Moore, D., Walker, J., \& Welm, S. (1993). Psychopharmacology Improved performance 4 hours after cocaine. Psychopharmacology, 110, 415-420.

Stokes, P. R. A., Mehta, M. a, Curran, H. V., Breen, G., \& Grasby, P. M. (2009). Can recreational doses of THC produce significant dopamine release in the human striatum? Neurolmage, 48, 186-90. doi:10.1016/j.neuroimage.2009.06.029

Stolberg, V. B. (2011). The use of coca: prehistory, history, and ethnography. Journal of ethnicity in substance abuse, 10, 126-46. doi:10.1080/15332640.2011.573310 
Talpos, J. C., Wilkinson, L. S., \& Robbins, T. W. (2006). A comparison of multiple 5-HT receptors in two tasks measuring impulsivity. Journal of Psychopharmacology, 20, 4758. doi:10.1177/0269881105056639

Toennes, S. W., Ramaekers, J. G., Theunissen, E. L., Moeller, M. R., \& Kauert, G. F. (2008). Comparison of cannabinoid pharmacokinetic properties in occasional and heavy users smoking a marijuana or placebo joint. Journal of analytical toxicology, 32, 470-7.

Toennes, S. W., Schneider, K., Kauert, G. F., Wunder, C., Moeller, M. R., Theunissen, E. L., \& Ramaekers, J. G. (2011). Influence of ethanol on cannabinoid pharmacokinetic parameters in chronic users. Analytical and bioanalytical chemistry, 400, 145-52. doi:10.1007/s00216-010-4449-2

Toennes, S. W., Steinmeyer, S., Maurer, H.-J., Moeller, M. R., \& Kauert, G. F. (2005). Screening for drugs of abuse in oral fluid--correlation of analysis results with serum in forensic cases. Journal of analytical toxicology, 29, 22-7.

Toker, L., Amar, S., Bersudsky, Y., Benjamin, J., \& Klein, E. (2010). The biology of tryptophan depletion and mood disorders. The Israel journal of psychiatry and related sciences, 47 , 46-55.

Trigo, J. M., Cabrero-Castel, A., Berrendero, F., Maldonado, R., \& Robledo, P. (2008). MDMA modifies active avoidance learning and recall in mice. Psychopharmacology, 197, 391400. doi:10.1007/s00213-007-1045-z

United Nations Office on Drugs and Crime. (2012). Cannabis: A Short Review. United Nations, Vienna.

Urban, N. B. L., Slifstein, M., Meda, S., Xu, X., Ayoub, R., Medina, O., ... Abi-Dargham, A. (2012). Imaging human reward processing with positron emission tomography and functional magnetic resonance imaging. Psychopharmacology, 221, 67-77. doi:10.1007/s00213-011-2543-6

Urban, N. B. L., Slifstein, M., Thompson, J. L., Xu, X., Girgis, R. R., Raheja, S., ... Abi-Dargham, A. (2012). Dopamine release in chronic cannabis users: a [11c] raclopride positron emission tomography study. Biological psychiatry, 71, 677-83. doi:10.1016/j.biopsych.2011.12.018

Van Dyke, C., Barash, P., Jatlow, P., \& Byck, R. (1976). Cocaine: plasma concentrations after intranasal application in man. Science, 191, 859-861.

Van Hell, H. H., Jager, G., Bossong, M. G., Brouwer, A., Jansma, J. M., Zuurman, L., ... Ramsey, N. F. (2012). Involvement of the endocannabinoid system in reward processing in the human brain. Psychopharmacology, 219, 981-90. doi:10.1007/s00213-011-2428-8

Van Wel, J. H. P., Kuypers, K. P. C., Theunissen, E. L., Bosker, W. M., Bakker, K., \& Ramaekers, J. G. (2011). Blockade of 5-HT(2) Receptor Selectively Prevents MDMA-Induced Verbal Memory Impairment. Neuropsychopharmacology, 36, 1-8. doi:10.1038/npp.2011.80 
Van Wel, J. H. P., Kuypers, K. P. C., Theunissen, E. L., Toennes, S. W., Spronk, D. B., Verkes, R. J., \& Ramaekers, J. G. (in press). Single doses of THC and cocaine decrease proficiency of impulse control in heavy cannabis users. British journal of pharmacology.

Van Wel, J. H. P., Kuypers, K. P. C., Theunissen, E. L., Bosker, W. M., Bakker, K., \& Ramaekers, J. G. (2012). Effects of acute MDMA intoxication on mood and impulsivity: role of the 5HT2 and 5-HT1 receptors. PloS one, 7, e40187. doi:10.1371/journal.pone.0040187

Verheyden, S. L., Hadfield, J., Calin, T., \& Curran, H. V. (2002). Sub-acute effects of MDMA (+/-3,4-methylenedioxymethamphetamine, "ecstasy") on mood: evidence of gender differences. Psychopharmacology, 161, 23-31. doi:10.1007/s00213-001-0995-9

Vermeeren, A., Jackson, J., Muntjewerff, N., Quint, P., Harrison, E., \& O'Hanlon, J. (1995). Comparison of acute alprazolam $(0.25,0.50$ and $1.0 \mathrm{mg})$ effects versus those of lorazepam $2 \mathrm{mg}$ and placebo on memory in healthy volunteers using laboratory and telephone tests. Psychopharmacology, 118, 1-9.

Verrico, C. D., Jentsch, J. D., \& Roth, R. H. (2003). Persistent and anatomically selective reduction in prefrontal cortical dopamine metabolism after repeated, intermittent cannabinoid administration to rats. Synapse, 49, 61-6. doi:10.1002/syn.10215

Volkow, N. D., Fowler, J. S., Wang, G. J., Baler, R., \& Telang, F. (2009). Imaging dopamine's role in drug abuse and addiction. Neuropharmacology, 56, 3-8. doi:10.1016/j.neuropharm.2008.05.022

Volkow, N. D., Fowler, J. S., Wang, G.-J., \& Swanson, J. M. (2004). Dopamine in drug abuse and addiction: results from imaging studies and treatment implications. Molecular psychiatry, 9, 557-69. doi:10.1038/sj.mp.4001507

Volkow, N. D., Wang, G.-J., Fowler, J. S., \& Tomasi, D. (2012). Addiction circuitry in the human brain. Annual review of pharmacology and toxicology, 52, 321-36. doi:10.1146/annurev-pharmtox-010611-134625

Volkow, N. D., Wang, G.-J., Fowler, J. S., Tomasi, D., \& Telang, F. (2011). Addiction: beyond dopamine reward circuitry. Proceedings of the National Academy of Sciences of the United States of America, 108, 15037-42. doi:10.1073/pnas.1010654108

Vollenweider, F. X., Studerus, E., Hasler, F., \& Paulus, M. (2006). Role of 5-HT1A receptors in the acute effects of MDMA on cognition and emotion in humans. International Journal of Neuropsychopharmacology, 9, S73.

Vollenweider, F. X., Liechti, M. E., Gamma, A., Greer, G., \& Geyer, M. (2001). Acute psychological and neurophysiological effects of MDMA in humans. Journal of psychoactive drugs, 34, 171-84.

Walsh, S. L., Stoops, W. W., Moody, D. E., \& Bigelow, G. E. (2010). Repeated dosing with oral cocaine in humans: Assessment of direct effects, withdrawal and pharmacokinetics. 
Experimental Clinical Psychopharmacology, 17, 205-216. doi:10.1037/a0016469.Repeated

Weinshenker, D., \& Schroeder, J. P. (2007). There and back again: a tale of norepinephrine and drug addiction. Neuropsychopharmacology 32, 1433-51. doi:10.1038/sj.npp.1301263

White, T. L., Lott, D. C., \& de Wit, H. (2006). Personality and the subjective effects of acute amphetamine in healthy volunteers. Neuropsychopharmacology 31, 1064-74. doi:10.1038/sj.npp.1300939

Wilens, T. E., \& Morrison, N. R. (2011). The intersection of attention-deficit/hyperactivity disorder and substance abuse. Current opinion in psychiatry, 24, 280-5. doi:10.1097/YCO.0b013e328345c956

Wilkinson, P., Dyke, C. Van, Jatlow, P., Barash, P., \& Byck, R. (1980). Intranasal and oral cocaine kinetics. Clinical Pharmacology \& Therapeutics, 27, 386-394.

Winterer, G., \& Weinberger, D. R. (2004). Genes, dopamine and cortical signal-to-noise ratio in schizophrenia. Trends in neurosciences, 27, 683-90. doi:10.1016/j.tins.2004.08.002

Yacubian, J., \& Büchel, C. (2009). The genetic basis of individual differences in reward processing and the link to addictive behavior and social cognition. Neuroscience, 164, 55-71. doi:10.1016/j.neuroscience.2009.05.015

Yasuno, F., Suhara, T., Nakayama, T., Ichimiya, T., Okubo, Y., Takano, A., ... Suzuki, K. (2003). Inhibitory Effect of Hippocampal 5-HT 1A Receptors on Human Explicit Memory. American Journal of Psychiatry, 160, 334-340.

Young, S. N., \& Leyton, M. (2002). The role of serotonin in human mood and social interaction. Insight from altered tryptophan levels. Pharmacology, biochemistry, and behavior, 71, 857-65.

Zanettini, C., Panlilio, L. V, Alicki, M., Goldberg, S. R., Haller, J., \& Yasar, S. (2011). Effects of endocannabinoid system modulation on cognitive and emotional behavior. Frontiers in behavioral neuroscience, 5, 1-21. doi:10.3389/fnbeh.2011.00057

Zuurman, L., Roy, C., Schoemaker, R. C., Hazekamp, a, den Hartigh, J., Bender, J. C. M. E., ... van Gerven, J. M. A. (2008). Effect of intrapulmonary tetrahydrocannabinol administration in humans. Journal of Psychopharmacology, 22, 707-16. doi:10.1177/0269881108089581 
SUMMARY 


\section{SUMMARY}

Cannabis, cocaine and MDMA are among the most used drugs of abuse in Europe. All of these drugs have been associated with acute impairments in impulse control and alterations in subjective experiences, while cannabis and MDMA have been shown to significantly impair memory. Cannabis, cocaine and MDMA exert their main effects through different neurotransmitter systems, but also share some actions on dopamine and serotonin transmission.

The dopaminergic system, which can be altered by cocaine or cannabis administration, has been shown to play a big role in impulse control, mood and psychedelic experiences. Therefore, a number of studies were designed to elucidate on these effects by the acute administration of cocaine and cannabis. It has previously been shown that increased impulsivity is connected to an increased likelihood of developing an addiction. Therefore it is useful to investigate how cannabis and cocaine intoxication influences impulsive responding after drug intake, since this could indicate how the use of a drug eventually leads to addiction. Furthermore, knowledge about the influence of trait impulsivity on subjective experiences after drug administration could be helpful in identifying persons sensitive to develop problematic drug use.

MDMA exerts its main effects through the serotonin system which has been implicated in impulsive responding, mood and memory. It is however not clear which serotonergic receptors are involved in these MDMA-induced effects. More knowledge about which serotonin receptors are involved in these functions is needed in order to effectively design drugs to ameliorate conditions associated with dysfunction of the serotonin system, such as major depressive disorder. Possible candidates are proposed to be the 5-HT 1 and 5$\mathrm{HT}_{2}$ receptors. Therefore, these receptors were targeted using a pre-treatment with $5-\mathrm{HT}_{1}$ and $5-\mathrm{HT}_{2}$ antagonists to investigate their role on memory, mood and impulsivity during MDMA intoxication.

The main goal of this dissertation was to investigate the effects of three drugs of abuse (cannabis, cocaine and MDMA) on impulsivity, mood and cognition. All studies described in this thesis used double-blind, placebo-controlled designs. In the first three chapters following the introduction, the influence of cannabis and cocaine on impulsive responding, mood and reward-related brain activity is described. The following two chapters 
elucidate the effect of MDMA on memory, impulsivity and mood, as well as the role of $5-\mathrm{HT}_{1}$ and $5-\mathrm{HT}_{2}$ receptors.

The study in chapter $\mathbf{2}$ was designed to assess whether the impairing effects of THC in heavy cannabis users would present in a wide range of neuropsychological functions or selectively pertain to specific performance domains. A second aim was to assess the acute effects of cocaine on neurocognitive functions of heavy cannabis users. 61 subjects received single doses of cocaine $(300 \mathrm{mg})$, cannabis $(300 \mu \mathrm{g} / \mathrm{kg}$ ) and placebo during 3 separate testing days and completed a number of tests measuring impulse control (Matching Familiar Figures Task, Stop-Signal task) and psychomotor function (Critical Tracking/Divided Attention Task, Tower of London Task). Results showed that single doses of cocaine improved psychomotor function and decreased response time in motor impulsivity tasks at a cost of making more errors. Heavy cannabis users displayed impairments in a broad range of neuropsychological domains during THC intoxication, including increased impulsive responding. The impairments observed in psychomotor tasks, but not in impulsivity tasks, appeared smaller in magnitude as compared to those previously reported in occasional cannabis users. This reduction in proficiency in impulse control after administration of cocaine and cannabis was speculated to put drug users at increased risk of repeated drug use and consequently addiction.

Chapter 3 set out to determine whether the subjective response to drugs differs between regular cannabis users who have normal or high levels of trait impulsivity. 122 subjects were administered cocaine (300 mg), cannabis $(450 \mu \mathrm{g} / \mathrm{kg}$ divided in two administrations) and placebo in 3 sessions. They completed a number of subjective questionnaires (Profile of Mood States, the Clinician Administered Dissociative States Scale, and the Bowdle visual analog scale) measuring mood, dissociative state, and psychedelic experience respectively. Trait impulsivity was assessed at baseline with the Barratt Impulsiveness Scale. Overall, cannabis intoxication increased ratings of dissociation and psychedelic state, as well as feelings of fatigue, confusion, depression and anxiety, and decreased feelings arousal, positive mood, vigor, friendliness and elation. Cocaine significantly increased ratings of dissociation, psychedelic state and feelings of vigor, friendliness, elation, positive mood, anxiety and arousal, while decreasing fatigue. Psychedelic experiences following cannabis 
use were most intense in high impulsive subjects. Also, feelings of dissociation following cocaine administration were also more intense in high impulsive subjects. It was concluded that trait impulsivity contributes to subjective experiences of drug use and increases the risk of developing drug use problems.

The study described in chapter 4 assesses the effects of two drugs (cannabis and cocaine) with opposing effects on the dopamine system on brain activity and behavioural performance related to reward anticipation as measured by the Monetary Incentive Delay task (MIDT). To this end, 50 healthy regular cannabis users received cocaine (300 $\mathrm{mg}), 2$ subsequent doses cannabis ( 350 and $150 \mu \mathrm{g} / \mathrm{kg}$ ) and placebo on 3 separate days. Subjects then performed the MIDT in the fMRI scanner. Both cannabis and cocaine administration significantly decreased thalamic activation as measured with BOLD response. On the behavioral level cocaine administration decreased reaction time as well as increasing the number of hits. Cannabis had the opposite effect, inducing slower reaction speed and a decrease in the number of hits. It was concluded that the differential activation of the indirect and direct thalamic pathways after cannabis and cocaine administration respectively explained the difference between the decrease in thalamic activation and opposing behavior following the drugs on the reward anticipation task. The decrease in activation of the thalamus following both drugs suggested a reduction in sensory gating, leading to an increase in striatal processing of sensory information and a reduction of higher cognitive control.

To further investigate the influence of drugs on memory, the research in chapter 5 focussed on the role of $5-\mathrm{HT}_{2 \mathrm{~A}}$ and $5-\mathrm{HT}_{1 \mathrm{~A}}$ receptors in MDMA-induced memory impairment. It was hypothesized that pretreatment with ketanserin, a $5-\mathrm{HT}_{2 \mathrm{~A}}$ receptor blocker, and pindolol, a 5- $\mathrm{HT}_{1 \mathrm{~A}}$ receptor blocker, would protect against MDMA-induced memory impairment. To this end, 17 subjects received placebo or MDMA $(75 \mathrm{mg})$ combined with pre-treatment of placebo, ketanserin $(50 \mathrm{mg}$ ) or pindolol $(20 \mathrm{mg})$ in 6 experimental sessions. Memory function was assessed by means of a Word Learning Task, a Spatial Memory Task and a Prospective Memory Task. MDMA significantly impaired performance in all memory tasks. Pretreatment with a $5-\mathrm{HT}_{2 \mathrm{~A}}$ receptor blocker selectively interacted with subsequent MDMA treatment and prevented MDMA induced impairment in the Word Learning Task, but not in the spatial and 
prospective memory task. Pretreatment with a $5-\mathrm{HT}_{1 \mathrm{~A}}$ blocker did not affect MDMA-induced memory impairment in any of the tasks. Together, the results demonstrate that MDMAinduced impairment of verbal memory as measured in the Word Learning Task is mediated by $5-\mathrm{HT}_{2 \mathrm{~A}}$ receptor stimulation.

The study in chapter 6 investigated whether pretreatment with $5-\mathrm{HT}_{1}$ and $5-\mathrm{HT}_{2}$ receptor blockers would prevent MDMA effects on mood and impulsivity using the same design as the study described in chapter 5. Subjects completed a Profile of Mood States questionnaire and several impulsivity tasks (Stop signal Task, Matching Familiar Figures Task, Cue Dependent Reversal Learning Task). MDMA alone increased both positive (vigor, arousal, friendliness, elation, positive mood) and negative affect (anxiety, confusion). Furthermore, MDMA administration increased response latency in the Stop Signal Task and the Matching Familiar Figures Task. Pretreatment with a 5- $\mathrm{HT}_{2 \mathrm{~A}}$ receptor blocker blocked MDMA effects on positive affect, but not negative affect but did not influence the effects of MDMA on impulsivity. A 5- $\mathrm{HT}_{1 \mathrm{~A}}$ receptor blocker did not interact with MDMA on any of the measures. It was concluded that $5-\mathrm{HT}_{2}$ receptors mediate positive moods induced by MDMA but not negative moods or impulsivity while $5-\mathrm{HT}_{1}$ receptors do not appear to be involved in MDMA effects on mood and impulse control.

Finally, in chapter $\mathbf{7}$ the results of the studies are discussed and integrated in a theoretical framework. First, the effects of cannabis and cocaine on impulsivity were discussed. Since cannabis and cocaine both affect impulsivity, but in opposing ways, it was suggested that treatment for addiction to one substance should be different than treatment for the other. For example, increased impulsive responding as seen after cocaine administration could point towards efficacy of GABA-agonists, while reducing the increased reflective impulsivity as seen after cannabis would be more suited to glutamate antagonists. In order to investigate how one goes from controlled drug use to addiction, a task measuring reward anticipation was employed. The thalamic deactivation seen after both cannabis and cocaine administration was hypothesized to be due to alterations in reward processing while using these drugs. These alterations in reward processing could eventually lead to an increased likelihood of developing an addiction. Another factor in developing addiction was proposed to be high trait impulsivity. It was shown that high trait impulsivity increased measures of 
dissociation and psychedelic experiences. Since previous studies have indicated a role for the involvement of $D_{2}$-receptors in increased trait impulsivity, it was suggested that the consequent hypersensitivity to dopamine increased the subjective responding of high impulsive subjects to both drugs. The effects of cannabis and cocaine on dissociation and psychedelic experiences were suggested to be due to alterations in the $D_{1} / D_{2}$ receptor ratio. Lastly, it was concluded that modulation of the $5-\mathrm{HT}_{2 \mathrm{~A}}$ receptor, but not the $5-\mathrm{HT}_{1}$ receptor, affects memory and mood. This suggests that addition of drugs that stimulate the $5-\mathrm{HT}_{2}$ receptor might be efficacious in the treatment of disorders such as PTSD or major depression.

In sum, the results from the studies described in this thesis invite thinking about novel approaches to addiction treatment. Additionally, ideas are presented for new research about the etiology and treatment of addiction and mood disorders. 
SAMENVATtING 


\section{SAMENVATtING}

Cannabis, cocaïne en MDMA behoren tot de drugs die het meest frequent worden gebruikt in Europa. Alle drie zijn ze geassocieerd met acute problemen met impulscontrole en veranderingen in subjectieve ervaringen. Daarnaast verslechteren cannabis en MDMA het geheugen significant. Deze cognitieve, psychomotore en subjectieve effecten van cannabis, cocaïne en MDMA worden veroorzaakt door de effecten van de drugs op verscheidene neurotransmitter systemen.

Het dopaminerge systeem, dat beïnvloed kan worden door toediening van cocaïne en cannabis, blijkt een grote rol te spelen in impulscontrole, stemming en psychedelische ervaringen. Om de acute effecten van cocaïne en cannabis op deze functies te verduidelijken werden een aantal studies uitgevoerd. Eerdere experimenten hebben laten zien dat verhoogde impulsiviteit gepaard gaat met een toegenomen risico op het ontwikkelen van een verslaving. Onderzoek naar de acute effecten van cocaïne en cannabis op impulsief gedrag kan een indicatie vormen van hoe het gebruik van een drug tot verslaving kan leiden. Kennis over de invloed van impulsiviteit als persoonlijkheidstrek op de subjectieve ervaringen na toediening van drugs kan nuttig zijn bij het identificeren van personen die gevoelig zijn voor het ontwikkelen van problematisch druggebruik.

MDMA beïnvloedt voornamelijk het serotonerge systeem. Het serotonerge systeem is betrokken bij impulsief reageren, stemming en geheugen. Het is echter nog niet duidelijk welke serotonine receptoren betrokken zijn bij deze effecten van MDMA. Deze kennis is nodig om medicijnen te kunnen ontwikkelen tegen stoornissen die geassocieerd worden met een disfunctioneel serotonine-systeem, zoals depressie. Eerder onderzoek heeft twee mogelijke kandidaten aangewezen, de $5-\mathrm{HT}_{1}$ en $5-\mathrm{HT}_{2}$ receptoren. Om de rol van deze receptoren op de effecten van MDMA op geheugen, stemming en impulsiviteit te testen werd een studie opgezet waarbij $5-\mathrm{HT}_{1}$ en $5-\mathrm{HT}_{2}$ antagonisten werden toegediend voordat een dosis MDMA werd gegeven.

Het doel van dit proefschrift was om het effect van drie drugs (cannabis, cocaïne en MDMA) op impulsiviteit, stemming en geheugen te onderzoeken. Alle studies die in deze these beschreven worden zijn dubbel-blind en placebo-gecontroleerd. In de eerste drie hoofdstukken volgend op de introductie wordt de invloed van cannabis en cocaïne op impulsief gedrag, stemming en hersenactiviteit beschreven. De twee hoofdstukken daarna 
verduidelijken de effecten van MDMA op geheugen, impulsiviteit en stemming en de rol van die de $5-\mathrm{HT}_{1}$ en $5-\mathrm{HT}_{2}$ receptoren hierbij spelen.

De studie beschreven in hoofdstuk 2 had als doel te onderzoeken of THC toediening bij regelmatige cannabis gebruikers verslechterende effecten had op meerdere neuropsychologische functies of dat deze beperkt bleven tot specifieke cognitieve domeinen. Een tweede doel van deze studie was het testen van acute effecten van cocaïne op het neurocognitief functioneren van regelmatige cannabis gebruikers. Hiertoe ontvingen 61 proefpersonen een enkelvoudige dosis cocaïne (300 mg), cannabis (300 $\mu \mathrm{g} / \mathrm{kg}$ ) en placebo op 3 afzonderlijke testdagen en deden ze een aantal taken die tot doel hadden impulscontrole (Matching Familiar Figures Taak, Stop-Signal taak) en psychomotorisch functioneren (Critical Tracking/Divided Attention Taak, Tower of London Taak) te meten. Resultaten wezen uit dat een enkele dosis van cocaïne het psychomotorisch functioneren verbeterde en de reactietijd verminderde op taken die motor impulsiviteit maten, terwijl daarbij meer fouten gemaakt werden. Regelmatige cannabisgebruikers lieten een verslechtering in functioneren zien op een verscheidenheid aan neuropsychologische functies, waaronder verhoogd impulsief gedrag. De verslechtering die werd gezien in psychomotorische taken, maar niet op impulsiviteitstaken, waren kleiner in grootte dan die in voorheen gerapporteerde studies met lichte cannabis gebruikers. Er werd gespeculeerd dat deze verslechtering in impulscontrole na toediening van cocaïne en cannabis ervoor zorgt dat druggebruikers een verhoogd risico hebben op herhaald druggebruik en verslaving.

In hoofdstuk 3 werd onderzocht of de subjectieve reactie op drugs verschilt tussen regelmatige cannabis gebruikers met een normaal of hoog niveau van impulsiviteit. 122 proefpersonen kregen cocaïne (300 mg), 2 opeenvolgende doses cannabis (350 en 150 $\mu \mathrm{g} / \mathrm{kg}$ ) of placebo toegediend tijdens drie sessies. Ze vulden een aantal subjectieve vragenlijsten in (Profile of Mood States, de Clinician Administered Dissociative States Scale, en de Bowdle visual analog scale) welke respectievelijk stemming, mate van dissociatie en psychedelische ervaring maten. Impulsiviteit als persoonlijkheidskenmerk werd gemeten met de Barratt Impulsiveness Scale. Cannabis en cocaïne verhoogden beide de mate van dissociatie, psychedelische ervaring en gevoelens van angst. Daarnaast waren onder invloed van cannabis gevoelens van moeheid, verwarring en depressie verhoogd, terwijl gevoelens 
van opgewondenheid, positieve stemming, energie, vriendelijkheid en opgetogenheid verlaagd waren. Cocaïne had een significant verhogend effect op gevoelens van energie, vriendelijkheid, opgetogenheid, positieve stemming en opgewondenheid, terwijl moeheid verminderd werd. Psychedelische ervaringen na cannabis gebruik waren het meest intens in hoog impulsieve personen. Tevens waren gevoelens van dissociatie na cocaïne toediening ook hoger in hoog impulsieve personen. Er werd geconcludeerd dat impulsiviteit als persoonlijkheidskenmerk een rol speelt in subjectieve ervaringen tijdens druggebruik en dat dit het risico op problematisch druggebruik zou kunnen verhogen.

In het onderzoek beschreven in hoofdstuk 4 werden de effecten van twee drugs (cannabis en cocaïne) met tegenovergestelde effecten op het dopamine-systeem onderzocht op hersenactiviteit en gedrag bij beloning. Hierbij werd gebruik gemaakt van de Monetary Incentive Delay taak (MIDT). Hiervoor ontvingen 50 gezonde, regelmatige cannabis gebruikers cocaïne (300 mg), 2 opeenvolgende doses cannabis (350 en $150 \mu \mathrm{g} / \mathrm{kg}$ ) of placebo in 3 afzonderlijke sessies. De proefpersonen deden vervolgens de MIDT in de fMRI-scanner. Zowel cannabis als cocaïne verlaagde significant de thalamische activiteit zoals gemeten door de BOLD-respons. Op gedragsniveau verlaagde cocaïne reactietijden en verhoogde het aantal goede antwoorden. Cannabis had het tegenovergestelde effect door de reactietijd te verhogen en het aantal goede antwoorden te verminderen. Er werd gesuggereerd dat een verschil in activiteit van de indirecte en directe thalamische paden na respectievelijk cannabis en cocaïne toediening kon verklaren waarom na toediening van beide drugs de thalamische activiteit was verlaagd, terwijl op gedragsniveau beide drugs tegenovergestelde effecten lieten zien. De vermindering in activiteit van de thalamus volgend op toediening van beide drugs suggereert een vermindering in 'sensory gating', wat leidt tot een verhoogde striatale verwerking van zintuiglijke signalen en een vermindering van cognitieve controle.

Om de effecten van drugs op geheugen verder te onderzoeken, richtte het onderzoek in hoofdstuk 5 zich op de rol van $5-\mathrm{HT}_{2}$ en $5-\mathrm{HT}_{1}$ receptoren bij geheugenstoornissen veroorzaakt door de toediening van MDMA. De hypothese was dat een voorbehandeling met ketanserine, een $5-\mathrm{HT}_{2}$ receptor-blokker, of pindolol, een medicijn dat de $5-\mathrm{HT}_{1}$ receptor blokkeert, bescherming zou bieden tegen geheugenstoornissen veroorzaakt door MDMA. Hiertoe ontvingen 17 proefpersonen placebo of MDMA (75 mg) in combinatie met een 
voorbehandeling met placebo, ketanserine $(50 \mathrm{mg})$ of pindolol $(20 \mathrm{mg})$ over 6 experimentele sessies. Geheugenfunctie werd getest met een woordenleertaak, een spatiële geheugentaak en een prospectieve geheugentaak. MDMA had een significant verslechterend effect op alle geheugentaken. Voorbehandeling met een $5-\mathrm{HT}_{2}$ receptorblokker vertoonde een significante interactie met de toediening van MDMA en voorkwam de verslechtering van het geheugen op de woordenleertaak na MDMA, maar had geen effect op de spatiële of prospectieve geheugentaak. Voorbehandeling met een $5-\mathrm{HT}_{1}$ blokker had geen invloed op de verslechtering van geheugen door MDMA-toediening. Tezamen laten deze resultaten zien dat de verslechtering van verbaal geheugen na toediening van MDMA zoals gemeten in de woorden leer taak gemedieerd wordt door stimulatie van de $5-\mathrm{HT}_{2}$ receptor.

De studie in hoofdstuk 6 onderzocht of voorbehandeling met $5-\mathrm{HT}_{1}$ en $5-\mathrm{HT}_{2}$ receptor blokkers de effecten van MDMA op stemming en impulsiviteit zou voorkomen. Hierbij werd dezelfde opzet gebruikt als in de studie beschreven in hoofdstuk 5. Proefpersonen vulden de Profile of Mood States vragenlijst in en voerden verschillende impulsiviteitstaken uit (Stop Signal Taak, Matching Familiar Figures Taak, Cue Dependent Reversal Learning Taak). MDMA alleen verhoogde zowel positief (energie, opgewondenheid, vriendelijkheid, opgetogenheid, positieve stemming) als negatief (angstigheid, verwardheid) affect. Verder verhoogde MDMA de stop-reactietijd in de Stop Signal Taak en de Matching Familiar Figures taak. Voorbehandeling met een 5- $\mathrm{HT}_{2}$ receptor blokker voorkwam dat MDMA een effect had op positief affect, maar niet op negatief affect. Het blokkeren van de $5-\mathrm{HT}_{2}$ receptor had eveneens geen invloed op de effecten van MDMA op impulsiviteitstaken. De 5- $\mathrm{HT}_{1}$ receptor blokker vertoonde op geen enkele maat interactie met MDMA. Hieruit werd geconcludeerd dat $5-\mathrm{HT}_{2}$ receptoren de positieve stemming die veroorzaakt wordt door MDMA mediëren, maar niet negatief affect of impulsiviteit, terwijl $5-\mathrm{HT}_{1}$ receptoren niet betrokken lijken te zijn bij de effecten van MDMA op stemming en impulscontrole.

Tot slot werden in hoofdstuk $\mathbf{7}$ de resultaten van de studies kritisch besproken en in een theoretisch raamwerk geïntegreerd. Eerst werden de effecten van cannabis en cocaïne op impulsiviteit besproken. Omdat zowel cannabis als cocaïne impulsiviteit beïnvloeden, maar op een tegengestelde manier, werd er gesuggereerd dat de behandeling voor verslaving aan de ene stof zou moeten verschillen van de behandeling voor verslaving aan de andere stof. 
Bijvoorbeeld, verhoogde impulsieve reacties na cocaïne toediening zouden kunnen wijzen op de effectiviteit van GABA-agonisten bij de behandeling voor cocaïne-verslaving, terwijl glutamaat-antagonisten meer geschikt zouden zijn voor het verminderen van verhoogde reflectieve impulsiviteit zoals gezien na cannabis toediening. Om te onderzoeken hoe men overgaat van incidenteel druggebruik naar verslaving werd een taak gebruikt die de verwachting van beloning mat. Er werd verondersteld dat aan de verminderde thalamus activiteit, die werd gezien na toediening van zowel cannabis als cocaïne, wijzigingen in de verwerking van beloning ten grondslag lagen. Dit zou uiteindelijk kunnen leiden tot een verhoogde kans op het ontwikkelen van een verslaving. Een andere factor die werd geopperd een rol te spelen in het ontwikkelen van een verslaving was impulsiviteit als karaktereigenschap. Er werd aangetoond dat personen met een hoge impulsiviteitscore een verhoogde mate van dissociatie en psychedelische ervaringen hadden. Omdat voorgaande studies een rol voor $\mathrm{D}_{2}$-receptoren bij verhoogde impulsiviteit als karaktertrek lieten zien, werd er gesuggereerd dat de daaropvolgende overgevoeligheid voor dopamine verantwoordelijk is voor de subjectieve reactie van hoog impulsieve personen na toediening van cannabis en cocaïne. Er werd gesuggereerd dat de effecten van cannabis en cocaïne op dissociatie en psychedelische ervaringen toe konden worden geschreven aan wijzigingen in de ratio van $D_{1} / D_{2}$ receptoren. Tot slot werd er geconcludeerd dat verandering in de $5-H T_{2}$ receptor, maar niet in de $5-\mathrm{HT}_{1}$ receptor, een effect heeft op geheugen en stemming. Dit suggereert dat de toevoeging van medicatie die de $5-\mathrm{HT}_{2}$ receptor stimuleert probaat zou kunnen zijn bij de behandeling van stoornissen zoals PTSS of depressie.

Kortom, de resultaten van de studies die beschreven worden in dit proefschrift zetten aan tot denken over een nieuwe benadering van de behandeling van verslaving. Bovendien worden er ideeën gepresenteerd die tot nieuw onderzoek over de etiologie en behandeling van verslaving en stemmingsstoornissen kunnen leiden. 


\section{DANKWOORD}


Na ruim 4 jaar hard werken is er een einde gekomen aan dit tijdperk. Voordat ik begon had ik nooit gedacht dat promoveren zo'n bevredigend en tegelijk frustrerend proces kon zijn! Terugkijkend op de afgelopen jaren heb ik veel steun gehad aan en plezier gehad met een heel aantal mensen, waarvan ik er ongetwijfeld een aantal zal vergeten. Daarom bedank ik bij voorbaat iedereen die zich op enige wijze verbonden voelt met dit proefschrift!

Ik wil uiteraard mijn promotor prof. Jan Ramaekers en mijn co-promotoren dr. Kim Kuypers en dr. Robbert Jan Verkes bedanken. Zonder hen had dit proefschrift niet bestaan. We hebben veel uren rond de tafel besteedt aan het schrijven en bijschaven van de papers en presentaties die uit het onderzoek van dit proefschrift zijn voortgevloeid. Dr. Eef Theunissen was het $4^{\mathrm{e}}$ wiel aan de wagen van mijn promotie; mede dankzij haar expertise en hulp heb ik het onderzoek tot een goed einde weten te brengen. Ik kon altijd bij jullie terecht met mijn vragen en problemen, ook al waren ze nog zo klein. Jullie hebben me altijd het gevoel gegeven dat ik welkom was.

Mijn dank gaat ook uit naar de leden van de beoordelingscommissie, prof. Wim Riedel, dr. Arjan Blokland, prof. Roshan Cools, prof. Bernhard Hommel en dr. Chantal Nederkoorn, voor het lezen en beoordelen van mijn proefschrift.

Mijn tijd als promovenda zou niet half zo leuk geweest zijn zonder alle andere collegapromovendi van de afdeling Neuropsychology \& Psychopharmacolohy. Silke, Tim, Peter, Stefan, Joep, Zoë, Eliza, Anke, Inge, Wendy, Alexandros en Joy, bedankt voor al het plezier op de afdeling en tijdens conferenties, de uitjes en de etentjes, maar ook voor alles wat ik van jullie heb kunnen leren.

Daarnaast wil ik ook nog alle andere collega's van NP\&PP bedanken, en in het speciaal de ondersteunening van Annemie en Elian bij al het regelwerk wat bij een promotie komt kijken, Anita voor het altijd klaarstaan met haar E-prime- en onderzoeksexpertise en Lisbeth en Peter voor hun onmisbare hulp met de fMRI-analyses. Ook de ICT-ondersteuning was onmisbaar tijdens het onderzoek, dus heren, bedankt! 
Desirée, mijn co-promovenda in Nijmegen voor het cannabis en cocaïne onderzoek. Bedankt voor alle gezellige tijden maar ook voor je suggesties voor het verbeteren van het onderzoek en de artikelen die daaruit voortvloeiden. Heel veel succes en plezier in Engeland, misschien ontmoeten we elkaar weer in Londen!

My special heartfelt thanks go out to my roommates, Luciana, Anne and Anique, who have helped me through many highs and lows in these 4 years. I will surely miss our discussions about food, life and politics! Aimée, bedankt voor alle gezellige uurtjes te paard en naast het paard. Luciana and Aimée, thanks for being my paranimfs. I couldn't have chosen better ones.

Tijdens het onderzoek doen werd mijn leven heel veel makkelijker gemaakt dankzij een aantal fantastische stagiaires en onderzoeksassistenten. Ruud en Justina, Carsten en Hatice, Renske en Eleanor, Kevin: ontzettend bedankt voor alle tijd en moeite die jullie in het onderzoek hebben gestoken. Zonder jullie had ik het onderzoek nooit op tijd kunnen afronden. Dit onderzoek uitvoeren zonder proefpersonen was onmogelijk geweest. Daarom gaat mijn dank ook uit naar alle proefpersonen die hebben meegedaan.

Mindy, ik wil jou ook heel erg bedanken voor het ontwerpen van mijn proefschrift. Het is echt ontzettend mooi geworden!

Naast alle mensen die direct of indirect verbonden waren aan mijn promotie, zijn er natuurlijk ook een aantal die er helemaal niks mee te maken hadden, maar die ik toch wil bedanken. Bedankt Mandy, Sophie, Josine en Annelies voor de afleiding van mijn werk in de vorm van gezellige shopdagen, high tea's, etentjes, weekendjes, avonden, vakanties en niet te vergeten het samen sporten!

Papa en mama, bedankt voor al jullie steun en opbeurende woorden. Het is fijn dat ik altijd mocht bellen of mailen als iets niet ging zoals ik wilde, als ik ergens over twijfelde of als ik ergens over jubelde. Jullie trots als een paper gepubliceerd werd of als ik weer ergens op congres ging was altijd voelbaar. Hopelijk kunnen jullie nog heel veel jaren achter me staan. 
Lieve Michiel, de afgelopen jaren heb je me altijd bijgestaan met je hulp, tips, heb je teksten voor me proefgelezen en ben je mijn proefkonijn geweest voor presentaties. Altijd stond je voor me klaar, natuurlijk niet alleen met praktische ondersteuning maar ook met liefde en geduld als ik weer eens het gevoel had dat het niet opschoot, dat analyses niet lukten zoals ik wilde, computerproblemen... Dank je wel! 


\section{CURRICULUM VITAE}

Janelle van Wel werd geboren op 26 oktober 1986 te Alkmaar. In 2004 behaalde zij haar VWO-diploma aan het Jan van Scorel-college aldaar. Vervolgens studeerde ze psychologie aan de universiteit van Maastricht. Na het behalen van haar bachelor diploma in 2007 vervolgde ze haar opleiding aan dezelfde universiteit met de master Neuropsychology. Als onderdeel daarvan liep ze stage bij de afdeling Neuropsychology \& Psychopharmacology aan de universiteit Maastricht. Tevens volgde ze nog enkele vakken aan de faculteit Gezondheidswetenschappen. Tussen december 2008 en augustus 2009 werkte ze als pharmacovigilance officer te InterdosPharma in Geleen. In augustus 2009 begon zij aan haar promotie-onderzoek naar de effecten van drugs op impulsiviteit, stemming en geheugen. Momenteel werkt ze als post-doc aan de universiteit van Antwerpen en doet zij onderzoek naar de relatie tussen druggebruik in de populatie en de concentraties van deze drugs in rioolwater. 
$160 \mid$ 


\section{Publications}

Van Wel, J. H. P., Kuypers, K. P. C., Theunissen, E. L., Toennes, S. W., Spronk, D. B., Verkes, R. J., \& Ramaekers, J. G. (2013). Single doses of THC and cocaine decrease proficiency of impulse control in heavy cannabis users. British journal of pharmacology, 170, 14101420.

Van Wel, J. H. P., Kuypers, K. P. C., Theunissen, E. L., Bosker, W. M., Bakker, K., \& Ramaekers, J. G. (2012). Effects of acute MDMA intoxication on mood and impulsivity: role of the 5$\mathrm{HT}_{2}$ and 5- $\mathrm{HT}_{1}$ receptors. PLoS One, 7, e401187. doi: 10.1371/journal.pone.0040187.

Van Wel, J. H. P., Kuypers, K. P. C., Theunissen, E. L., Bosker, W. M., Bakker, K., \& Ramaekers, J. G. (2011). Blockade of 5- $\mathrm{HT}_{2}$ Receptor Selectively Prevents MDMA-Induced Verbal Memory Impairment. Neuropsychopharmacology, 36, 1-8. doi:10.1038/npp.2011.80

Spronk D.B., van Wel J.H.P., Ramaekers J.G., Verkes R.J. (2013). Characterizing the cognitive effects of cocaine: a comprehensive review. Neuroscience \& Biobehavioral Reviews, 37, 1838-1859.

\section{Submitted}

van Wel J.H.P, Kuypers K.P.C., Theunissen E.L., Toennes S.W., Spronk, D.B., Verkes R.J., Ramaekers J.G. Trait impulsivity contributes to psychedelic experience of cannabis and cocaine use.

Wille, S.M.R., Di Fazio, V., Toennes, S.W., van Wel, J.H.P., Ramaekers, J.G., Samyn, N. (under review). Evaluation of $\Delta^{9}$-tetrahydrocannabinol detection using Drugwipe $5 S^{\otimes}$ screening and oral fluid quantification after Quantisal ${ }^{\mathrm{TM}}$ collection for roadside drug detection purposes via a controlled study with chronic cannabis users. Drug Testing and Analysis.

van Heugten - Van Der Kloet, D., Giesbrecht, T., van Wel, J.H.P., Bosker, W.M., Kuypers, K.P.C., Theunissen, E.L, Spronk, D.B., Verkes, R.J., Merckelbach, H., Ramaekers, J.G. (submitted). MDMA, Cannabis, and Cocaine Produce Acute Dissociative Symptoms. Journal of Neurology, Neurosurgery, and Psychiatry.

In preparation

van Wel J.H.P., Kuypers K.P.C., Theunissen E.L., Toennes S.W., Evers, E.A., Spronk, D.B., Verkes R.J., Stiers, P., Ramaekers J.G. (in preparation). Reward related brain activity and task performance during cannabis and cocaine intoxication.

Spronk, D.B., De Bruijn, E.R.A, Roelofs, K., Muskens, J., van Wel, J.H.P., Ramaekers, J.G. \& Verkes, R.J. Acute effects of cannabis on social action tendencies as a function of Behavioral Inhibition System. 